\title{
PARADIFFERENTIAL OPERATORS AND CONORMAL DISTRIBUTIONS
}

\author{
Dissertation \\ zur Erlangung des mathematisch-naturwissenschaftlichen Doktorgrades \\ "Doctor rerum naturalium" \\ der Georg-August-Universität Göttingen \\ im Promotionsprogramm Mathematical Science \\ der Georg-August University School of Science (GAUSS)
}

vorgelegt von

Robin Spratte

aus Korbach.

Göttingen, 2019 
Betreuungsausschuss

Professor Dr. Ingo WitT,

Mathematisches Institut,

Georg-August-Universität Göttingen

Professor Dr. Dorothea Bahns,

Mathematisches Institut,

Georg-August-Universität Göttingen

Mitglieder der Prüfungskommission

Referent: Professor Dr. Ingo FRANK WiTt

Korreferent: Professor Dr. Dorothea Bahns

Weitere Mitglieder der Prüfungskommission

Professor Dr. JÖRG BRÜDERN,

Mathematisches Institut,

Georg-August-Universität Göttingen

Professor Dr. Chengchang Zhu,

Mathematisches Institut,

Georg-August-Universität Göttingen

Professor Dr. Dominik Schuhmacher, Institut für Mathematische Stochastik,

Georg-August-Universität Göttingen

Jun.-Professor Dr. Christoph Lehrenfeld,

Institut für Numerische und Angewandte Mathematik,

Georg-August-Universität Göttingen

Tag der mündlichen Prüfung: 28.10.2019 
Für Elias. 



\section{Danksagungen}

Mein erster Dank gilt Ingo Witt, der mein Verständnis von PDE's und Pseudifferentialoperatoren im Speziellen, aber auch der Mathematik im Allgemeinen in zahlreichen Gesprächen ganz wesentlich geprägt hat. Ich bin dankbar dafür, während fast der gesamten Zeit meines Studiums in ihm einen Lehrer und Ratgeber gefunden zu haben, der auf meine teils sprunghaften Gedankengänge eingehen konnte und mir dabei immer wieder neue Aspekte aufzeigte. Zu eigentlich jeder Frage konnte er bereits nach kurzem Stöbern ein bis zwei weitere Bücher auftun, wenn mein Wissenshunger noch nicht gestillt war.

Mein besonderer Dank gilt auch Dorothea Bahns, die sich über die Förderung meines Interesses an der mathematischen Physik hinaus als Sprecherin des GRK 1493 für meine finanzielle Förderung einsetzte und eine Verlängerung des Stipendiums in Elternschaft trotz einiger Widrigkeiten problemfrei organisierte. Bei allen möglichen Fragen fand ich bei ihr ein offenes Ohr und bin für ihre Förderung und Unterstützung sehr dankbar.

Danken möchte ich auch meinen Kommilitonen und Freunden Malte Heuer, Thorsten Groth und Matthias Krüger. Auch wenn Malte und Thorsten meine Vorliebe für die Analysis nicht gänzlich teilten, waren sie mir doch Zuhörer und Frustrationsfänger, wenn ich mich mal wieder in einer Sackgasse sah. Dankbar bin ich auch für die sprachlichen und $\mathrm{T}_{\mathrm{EX}}$-nischen Hilfestellungen der beiden für diese Arbeit. Mit Matthias verbindet mich neben der gemeinsamen Zeit auf Konferenzen, in Vorlesungen, Seminaren und Veranstaltungen des GRKs auch eine Leidensgemeinschaft der anfänglichen Verständnisschwierigkeiten von Operatoren und Symbolen vom Typ $(1,1)$, die sich zum Glück schnell auflösten.

In meinem Promotionsstudium wurde ich im Rahmen des Graduiertenkollegs 1493 über ein Stipendium der Deutschen Forschungsgemeinschaft finanziert. Ich bin allen PI's des GRK und der DFG dankbar, sowohl für die Organisation des Kollegs und seiner Veranstaltungen als auch für die finanzielle Förderung, die mir gleichzeitig ein fokussiertes Studium und fachliche Breite ermöglichten.

Meine Kinder sind mir eine stetige Quelle der Freude. Ihr Lachen und ihre Unbeschwertheit haben mir auch schwere Tage erleichtert und lassen mich stets nach vorne schauen. Nicht zuletzt wäre diese Arbeit niemals entstanden ohne die beständige Liebe und Unterstützung meiner Frau. Ich bin unendlich dankbar, mein Leben in Allem mit ihr teilen zu dürfen und möchte keinen Tag mit ihr missen. 

Have you not known? Have you not heard? The Lord is the everlasting God, the Creator of the ends of the earth. He does not faint or grow weary; his understanding is unsearchable.

Isiah 40:28, English Standard Version 



\section{Contents}

$\begin{array}{ll}\text { Chapter 1. Introduction } & 11\end{array}$

$\begin{array}{ll}\text { 1. Propagation of singularities } & 11\end{array}$

$\begin{array}{lll}\text { Chapter 2. Conormal Distributions of type }(1,1) & 15\end{array}$

1. Symbol Spaces 15

2. Pseudodifferential operators of type $(1,1) \quad 23$

3. Products, Paraproducts and the Taylor formula 26

4. Non-linear superposition 36

$\begin{array}{lll}\text { Chapter 3. The Linear Cauchy Problem } & 51\end{array}$

1. Transport equation 51

2. First order equation 53

3. The reduced problem $\quad 55$

Chapter 4. Nonlinear Propagation of Conormality 61

$\begin{array}{lll}\text { Chapter 5. Outlook } & 63\end{array}$

$\begin{array}{ll}\text { Appendices } & 65\end{array}$

$\begin{array}{lll}\text { Chapter A. Littlewood Paley decomposition and Besov spaces } & 67\end{array}$

1. Hölder-Zygmund estimates 69

$\begin{array}{ll}\text { Bibliography } & 71\end{array}$ 



\section{CHAPTER 1}

\section{Introduction}

In this thesis we develop a generalization of Hörmander's symbol calculus of conormal distributions $[\mathbf{H o ̈ 0 7}$, Chapter 18.2] and provide techniques for applications to nonlinear hyperbolic Partial Differential Equations. In particular we will provide explicit expansion formulas for symbols of conormal distributions under multiplication (Theorem 2.17 and Theorem 2.19) and nonlinear superposition with HölderZygmund continuous functions (Theorem 2.40).

We also define the class of diffeomorphisms of conormal type and establish their structure as a group (Theorems 2.29 and 2.42), again giving explicit expansion formulas for their symbols. This enables us to define conormal distributions with respect to non smooth hypersurfaces endowed with the established symbol calculus.

The definitions we give and the methods we develop are applicable to nonlinear Partial Differential Equations. In Chapter 3 we explicitly construct approximate symbolic solutions to a Cauchy problem with coefficients and datum given as conormal distributions. We obtain solvability of the reduced problem within a sufficiently smooth remainder space. In Chapter 4 we provide propagation of conormality for the developed symbolic calculus under hyperbolic quasilinear equations of first order.

Therefore the main result of this thesis is the 'correct' construction of this generalization of conormal distributions with respect to non smooth hypersurfaces and the provided methods. Further the computational results of the symbol calculus, especially the symbol expansion formulas for multiplication, composition and nonlinear superposition can be directly applied to Hörmander's symbol calculus of conormal distributions.

\section{Propagation of singularities}

Linear Theory. Studying propagation of singularities, the question is, given the location and type of singularities of the solution at an initial Cauchy surface or in the past, can we determine the location and type of singularities in the future. Take for example the Klein-Gordon equation

$$
\left(\square+\mu^{2}\right) u=\partial_{t}^{2} u-\sum_{j=1}^{n} \partial_{x_{j}}^{2} u+\mu^{2} u=0
$$

- a simple prototype for linear strictly hyperbolic Partial Differential Equations of second order. It is well known, that this equation has finite propagation speed. Meaning, if the Cauchy data at $t=0$ are supported in the point $x=0$, the solution will be supported inside the closed forward light cone

$$
\bar{V}_{+}=\left\{(t, x) \in \mathbb{R}_{\geq 0} \times \mathbb{R}^{n} ; t \geq|x|\right\},
$$


which by linearity of the equation yields the full picture of the spread of support and in general can't be improved. The singular support on the other hand does only propagate along the boundary of the light cone

$$
\partial V_{+}=\left\{(t, x) \in \mathbb{R}_{\geq 0} \times \mathbb{R}^{n} ; t=|x|\right\},
$$

which means singularities somehow keep track of their 'direction'. And the singular support is insufficient to describe the propagation of singularities.

The information of 'direction' is best included by the wave front set

$$
\mathrm{WF}(u) \subseteq T^{*} \mathbb{R}^{n+1} \backslash\{0\},
$$

which carries additional information to the singular support. Geometrically it is a closed conical subset of the cotangent bundle with the zero section removed. It can be defined as 'directions', in which the Fourier transform of local smooth cutoffs does not rapidly decrease. It carries more information, because the singular support can be obtained from the wave front set, simply by projecting to the spacial variable.

To study the propagation of singularities in a general situation one further needs the principal symbol

$$
\begin{aligned}
P & =\sum_{|\alpha| \leq m} a_{\alpha}(x) \partial_{x}^{\alpha} \\
\sigma^{m}(P)(x, \xi) & =\sum_{|\alpha|=m} a_{\alpha}(x)(i \xi)^{\alpha}
\end{aligned}
$$

of the operator. Then the theorem of propagation of singularities of Hörmander [DH72 $]$ implies that the wave front set of a solution

$$
P u=0
$$

propagates along the null bi-characteristic lines in the cotangent bundle. This means, that first the wave front set is a subset of the characteristic set of $P$

$$
\operatorname{Char}(P)=\left\{(x, \xi) \in T^{*} \mathbb{R}^{n+1} \backslash\{0\} ; \sigma^{m}(P)(x, \xi)=0\right\} .
$$

And second the wave front set is invariant under the bi-characteristic flow, induced by the Hamiltonian vector field $H_{\sigma^{m}(P)}$ on the symplectic manifold $T^{*} \mathbb{R}^{n+1} \backslash\{0\}$. There are also quantitative versions of this theorem that the singularities do not get worse in some sense.

Nonlinear theory. In the late 1970's the propagation of singularities for semilinear equations, especially wave equations

$$
\square u=f(u, \nabla u)
$$

was studied by Bony, Rauch, Reed, Beals and others. Early results by Reed [Ree78. showed that in the case of one space dimension the semilinear wave equation still reflects the linear case and singularities only propagate on the boundary of the light cone. Reed used elementary methods relying on the special structure of the wave operator with one space dimension.

Rauch showed in $[\mathbf{R a u 7 9}\rfloor$ that in higher space dimension additional singularities would appear if two singularities on different rays would cross, emitting new singularities of higher regularity onto the entire boundary of the light cone from the point of crossing. This type of phenomenon was coined interaction.

In a joint paper $[\mathbf{R R 8 0}]$ Rauch and Reed again analyzed the case of one spacial dimension. They concluded that the propagation of singularities result for the linear 
case was insufficient for this application. They consider the example of an inhomogenous Cauchy problem,

$$
\left\{\begin{array}{l}
\square u=g \\
u(0, x)=u_{1}(x), \quad \partial_{t} u(0, x)=u_{2}(x)
\end{array}\right.
$$

given Cauchy data $\left(u_{1}, u_{2}\right)$ supported in $[-1,1]$ of some regularity and inhomogeniety $g \in C^{\infty}$. The solution $u$ then satisfies on the half strip $t+1 \geq x \geq t-1$, for $t>2$ that

$$
\left(\partial_{t}+\partial_{x}\right)^{j} u \in C^{\infty} \quad \forall j>0 .
$$

This implies the previous result of the linear theory stated in terms of the wave front set, but indeed is (much) stronger, even if we were to replace $C^{\infty}$ by say $H_{\text {loc }}^{s}$. Especially by chain and product rule the class of such functions $(s>1)$ is invariant under nonlinear superposition $f(u)$, given $f \in C^{\infty}$, and multiplication. This is unlike the space of functions with restrictions on their wave front set.

They gave a rigorous definition of this concept, where $\left(\partial_{t}+\partial_{x}\right)$ is replaced by a characteristic vector field $\gamma$ - which means a non vanishing vector field, normal to one of the characteristics of the operator. They also replaced $C^{\infty}$ by $B$, any fixed suitable Banach space.

$$
B_{\gamma}^{\ell}=\left\{u \in B ; \gamma^{j} u \in B, \quad \forall 0 \leq j \leq \ell\right\}
$$

They proved that singularities of this type do propagate for linear second order hyperbolic operators in one spacial dimension. Using this they proved the earlier result of Reed for general semilinear second order hyperbolic equations. They also disproved it for higher order equations, constructing an interaction, where a new singularity was emitted onto a third characteristic. They were able to show, that apart from such interaction terms, no new singularities do occur in one spacial dimension.

Bony expanded on their ideas and gave the following definition of conormal distributions in $\left[\right.$ Bon80]. He defined the space $H^{s, \ell}(\Sigma)$ for closed sets $\Sigma \subseteq \mathbb{R}^{n+1}$ - typically a union of characteristic surfaces - to be

$$
u \in H^{s, \ell}(\Sigma) \Leftrightarrow X_{1} \cdots X_{j} u \in H^{s}
$$

for $0 \leq j \leq \ell$ and $X_{1}, \ldots, X_{j}$ vector fields parallel to $\Sigma$.

This proved to be a very applicable space for the study of semilinear hyperbolic equations, since as $B_{\gamma}^{\ell}$ it is invariant under nonlinear superposition and multiplication, but unlike $B_{\gamma}^{\ell}$ not restricted to the case of one spacial dimension. ${ }^{1}$

In subsequent papers of Melrose and Ritter [MR85], Bony [Bon86 and other authors it was established that in general dimension and for general order of the equation conormal singularities do propagate in some sense. Namely, it was shown that only new singularities from interaction with controlled regularity will occur. This was found to be false for general singularities. Beals showed in Bea83, that in space dimensions $n>1$ unless the initial data is a conormal distribution the singular support of a solution will in general fill the entire forward light cone of the singular support of the initial data and not only its boundary. This phenomenon

\footnotetext{
${ }^{1}$ Only in one spacial dimension will the characteristic set have discrete directions and only in a two dimensional space will a covector correspond to a tangent vector by conormality.
} 
was coined self-spreading and further showed that conormality as defined in (1) is a strong restriction.

Symbolic approach in linear theory. Hörmander pointed out, that slightly altering the definition in (1) - namely $H^{s}$ needs to be replaced by $B_{2, \infty}^{s}$ - allows for a different characterization in the special case $\ell=\infty$ and $\Sigma$ a hypersurface of codimension $k$. Then there is a representation of $u$ locally near $\Sigma$ as an oscillatory integral. That means away from $\Sigma u$ would be smooth and for suitable coordinates $x=\left(x^{\prime \prime}, x^{\prime}\right)$ we have in a neighborhood of $\Sigma$

$$
u(x)=\int e^{i x^{\prime} \xi^{\prime}} a\left(x, \xi^{\prime}\right) \mathrm{d} \xi^{\prime}
$$

with $a \in S_{1,0}^{-s-k / 2}$ a symbol of type $(1,0)$. See for example [Hö07, Lemma 18.2.4]. Hörmander's notation for this space is $I^{\mu}(\Omega, \Sigma)$ with $\mu=-s-k / 2+n / 4$. This very precise symbolic representation uncovers a much richer structure of conormal distributions, especially one obtains the principal symbol map

$$
0 \rightarrow I^{\mu-1}(\Omega, \Sigma) \rightarrow I^{\mu}(\Omega, \Sigma) \rightarrow S_{1,0}^{-s-k / 2}\left(\mathbb{R}^{n}, \mathbb{R}^{k}\right) / S_{1,0}^{-s-k / 2-1}\left(\mathbb{R}^{n}, \mathbb{R}^{k}\right) \rightarrow 0 .
$$

This principal symbol map coincides with the principal symbol for pseudodifferential operators, as the kernel of a pseudodifferential operator is a conormal distribution with respect to the diagonal. The parameter shift of Hörmander is constructed such that the kernel of an operator in $\Psi^{m}(\Omega)$ lies in $I^{m}(\Omega \times \Omega, \Delta)$, with $\Delta=\{(x, x) \mid x \in$ $\Omega\}$.

With principal symbols we also have a symbol expansion for pseudodifferential operators acting on conormal distributions similar to the composition of pseudodifferential operators.

$$
\begin{aligned}
p(x, D) u & =\int e^{i x^{\prime} \xi^{\prime}} b\left(x^{\prime \prime}, \xi^{\prime}\right) \mathrm{d} \xi^{\prime} \\
b & \sim \sum\left(\left\langle i D_{x^{\prime \prime}}, D_{\xi^{\prime \prime}}\right\rangle-\left\langle i D_{x^{\prime}}, D_{\xi^{\prime}}\right\rangle\right)^{j} p(x, \xi) a\left(x^{\prime \prime}, \xi^{\prime}\right) / j !_{\xi^{\prime \prime}=x^{\prime}=0}
\end{aligned}
$$

Thus especially the resulting principal symbol is the product of the operator symbol and the symbol of the conormal distribution, restricted to the respective cotangent bundle. In the case of a hyperbolic operator, we obtain a vanishing first order term on any characteristic cotangent bundle. Here the principal symbol is given by the Poisson bracket of the initial symbols, yielding the transport equation for the principal symbol.

Using the transport equation and suitable initial conditions one can explicitly solve the equation on a symbolic level up to an arbitrarily smooth remainder term. 


\section{CHAPTER 2}

\section{Conormal Distributions of type $(1,1)$}

In this chapter we will introduce a type of distributions governed by type $(1,1)$ symbols. We will learn that these distributions naturally lie in certain Besov spaces. We will enhance the structure by an improved smoothness, which will yield the principal behavior of the distributions. We will obtain a symbol expansion for multiplication, non-linear superposition and operator actions in such principal terms up to a remainder space of Besov spaces.

Based on these computations we will give a structure of such distributions with respect to non-smooth hypersurfaces. This will be a preparation for our applications to linearized Cauchy problems and quasilinear equations.

\section{Symbol Spaces}

First we are going to give a preliminary definition of the symbols in use to ease the understanding of the underlying structures. They will already fully describe the spaces of distributions that we are interested in. We will later amend the definition of symbols by an anisotropic structure, which will become handy for computations.

1.1. Basic Definitions. In the following we will use the notation $x=\left(x^{\prime \prime}, x^{\prime}\right) \in$ $\mathbb{R}^{n-k} \times \mathbb{R}^{k}$, where $k$ will be called the codimension. We are going to define the symbol space $S_{1,1}^{m, \rho}$, the remainder space $G^{m-\rho, \ell}$ and the space of conormal distributions $I_{1,1 ; \ell}^{m, \rho}$ as a combination of a symbolic part defined by an oscillatory integral and a remainder function.

Definition 2.1 (Smooth Symbol classes of type $(1,1)$ ). Let $a \in C^{\infty}\left(\mathbb{R}^{n} \times \mathbb{R}^{k}\right)$, then $a \in S_{1,1}^{m, \rho}\left(\mathbb{R}^{n} \times \mathbb{R}^{k}\right)$ with $m \in \mathbb{R}$ and $\rho>0$, if

$$
\begin{aligned}
\left|\partial_{x}^{\alpha} \partial_{\eta^{\prime}}^{\beta} a\left(x, \xi^{\prime}\right)\right| & \lesssim\left\langle\eta^{\prime}\right\rangle^{m+(|\alpha|-\rho)^{+-}-|\beta|} \quad \forall|\alpha| \neq \rho, \forall \beta \\
\left\|\partial_{\eta^{\prime}}^{\beta} a\left(\cdot, \eta^{\prime}\right)\right\|_{C_{*}^{\rho}} & \lesssim\left\langle\eta^{\prime}\right\rangle^{m-|\beta|} .
\end{aligned}
$$

Here $C_{*}^{s}$ is the Hölder-Zygmund space as defined in Chapter A. $a$ will be called a symbol of order $m$ with improved regularity $m-\rho$. If $a$ however only fulfills

$$
\left|\partial_{x}^{\alpha} \partial_{\eta^{\prime}}^{\beta} a\left(x, \xi^{\prime}\right)\right| \lesssim\left\langle\eta^{\prime}\right\rangle^{m+|\alpha|-|\beta|} \quad \forall \alpha, \beta .
$$

then $a \in S_{1,1}^{m}\left(\mathbb{R}^{n} \times \mathbb{R}^{k}\right)$. In an abuse of notation for $\rho \leq 0$ we will denote $S_{1,1}^{m, \rho}=$ $S_{1,1}^{m-\rho}$.

Definition 2.2. Let $m \in \mathbb{R}$ and $\ell \in \mathbb{N}$, then define the remainder space $G^{\mu, \ell}$ with respect to codimension $k$ as

$$
\begin{aligned}
G^{m, 0} & =\bigcap_{1<p \leq \infty} \text { unif } B_{p, \infty}^{-m-k(1-1 / p)}\left(\mathbb{R}^{n}\right) \\
G^{m, \ell} & =\left\{u \in G^{m, 0} ;\left(x^{\prime}\right)^{\alpha^{\prime}} u \in G^{m-\left|\alpha^{\prime}\right|}, \forall 0<\left|\alpha^{\prime}\right| \leq \ell\right\} .
\end{aligned}
$$


Here unif $B_{p, \infty}^{s}\left(\mathbb{R}^{n}\right)$ is the uniform Besov space as defined in Chapter A. We will write $G^{m}$ for $G^{m, 0}$ and $G^{m, \infty}$ for $\cap_{\ell \in \mathbb{N}} G^{m, \ell}$.

The distributions that we consider will be defined using symbols of type $(1,1)$ with improved regularity.

Definition 2.3. We call a distribution $u \in \mathcal{S}^{\prime}\left(\mathbb{R}^{n}\right)$ a conormal distribution of type $(1,1)$ and order $(m, \rho)$ with localization $\ell \in \mathbb{N}_{0}$, write $u \in I_{1,1 ; \ell}^{m, \rho}\left(\mathbb{R}^{n}, \mathbb{R}^{n-k}\right)$, for $m-$ $\rho+k<0$ if there is a decomposition $u=u_{C}+u_{G}$ and a symbol $a \in S_{1,1}^{m, \rho}\left(\mathbb{R}^{n-k} \times \mathbb{R}^{k}\right)$ such that $u_{G} \in G^{m-\rho, \ell}$ and

$$
u_{C}(x)=\int e^{i\left\langle x^{\prime}, \eta^{\prime}\right\rangle} a\left(x^{\prime \prime}, \eta^{\prime}\right) \mathrm{d} \eta^{\prime} .
$$

Then $a$ is called a full symbol of $u$ and it is called a total symbol of $u$ if $u_{G}=0$. If $u$ is given by a total symbol, we call $u$ fully symbolic. We will denote $I_{1,1 ; 0}^{m, \rho}$ as $I_{1,1}^{m, \rho}$. For more consistency of notation we will sometimes denote $G^{m}$ as $I_{1,1}^{m}$.

First we state an immediate consequence of the symbol definition

Proposition 2.4. For all $|\alpha| \neq \rho$ we have

$$
\begin{aligned}
\partial_{x}^{\alpha} S_{1,1}^{m, \rho} & \subseteq S^{m, \rho-|\alpha|} \quad \forall|\alpha| \neq \rho \\
I_{1,1}^{m-\delta, \rho-\delta} & \subseteq I_{1,1}^{m, \rho} \quad \forall \delta \geq 0
\end{aligned}
$$

Proof. The assertions follow from the fact that $\partial_{x}^{\alpha}$ maps $B_{p, \infty}^{\rho}$ to $B_{p, \infty}^{\rho-|\alpha|}$.

REMARK 2.5. As $u_{C}$ is defined as the partial anti Fourier transform of a symbol, the integral in (1) is always well defined, independent of the condition $m-\rho+k<0$. But we want our definition to be stable if one also considers $x^{\prime}$-dependent symbols. To understand why $m-\rho+k<0$ is then necessary, consider the following 'standard' pathological example of a symbol in $S_{1,1}^{0}$, given by Hörmander in $[\mathbf{H o ̈ 0 3 b}$ and closely related to counterexamples due to Ching [Chi72] and Bourdaud [Bou88].

Let $A \in C_{c}^{\infty}\left(B_{1 / 2}(1)\right)$, then

$$
a\left(x^{\prime}, \eta^{\prime}\right)=\sum_{\nu \geq 0} e^{-i 2^{\nu} x^{\prime}} A\left(2^{-\nu} \eta^{\prime}\right) \in S_{1,1}^{0} .
$$

If we were to formally define $u_{C}$ via (1), then we would obtain as Fourier transform

$$
\begin{aligned}
\hat{u}_{C}\left(\xi^{\prime}\right) & =\int \hat{a}\left(\xi^{\prime}-\eta^{\prime}, \eta^{\prime}\right) \mathrm{d} \eta^{\prime}=\sum_{\nu} \int \delta\left(\xi^{\prime}-\eta^{\prime}+2^{\nu}\right) A\left(2^{-\nu} \eta^{\prime}\right) \mathrm{d} \eta^{\prime} \\
& =\sum_{\nu} A\left(1+2^{-\nu} \xi^{\prime}\right)
\end{aligned}
$$

yielding a sum not converging in $\mathcal{S}^{\prime}(\mathbb{R})$ if $A(1) \neq 0$.

These effects are parallel to the problematic twisted diagonal in Hörmander's approach to paradifferential operators and their kernels. For operators this leads to noncontinuity on $H^{s}$ for $s \leq 0$, for this model of conormal distributions this leads to complete ill-definedness of (1). 
1.2. Besov space embeddings. In order to simplify notation, especially to avoid case separations when taking spacial derivatives, we introduce the following notation for some $\epsilon>0$

$$
(s)_{\epsilon}^{+}= \begin{cases}s & s \geq \epsilon \\ \epsilon & \epsilon \geq s \geq 0 \\ (s+\epsilon)^{+} & s<0 .\end{cases}
$$

We also introduce

$$
\begin{array}{ccc}
(s)_{\epsilon}^{-}=(s)_{\epsilon}^{+}-s & \Rightarrow & s=(s)_{\epsilon}^{+}-(s)_{\epsilon}^{-} \\
(s)_{\epsilon}^{\Delta}=(\epsilon-|s|)^{+} & \Rightarrow & (s)_{\epsilon}^{+}=s^{+}+(s)_{\epsilon}^{\Delta} .
\end{array}
$$

Estimates involving such terms are to be understood as being uniform with constants only depending on $\epsilon>0$.

For the technical analysis of our distribution spaces, we need to introduce a slightly weaker version of our symbol spaces.

Definition 2.6. Let $a \in C_{*}^{r}\left(\mathbb{R}^{n} \times \mathbb{R}^{k}\right)$, then $a \in C_{*}^{r} S_{1,1}^{m}$ iff

$$
\begin{aligned}
\left|\partial_{\eta^{\prime}}^{\beta} a\left(x, \eta^{\prime}\right)\right| & \lesssim\left\langle\eta^{\prime}\right\rangle^{m-|\beta|} \\
\left\|\partial_{\eta^{\prime}}^{\beta} a\left(\cdot, \eta^{\prime}\right)\right\|_{C_{*}^{s}} & \lesssim\left\langle\eta^{\prime}\right\rangle^{m-|\beta|+s} \quad \forall 0 \leq s \leq r
\end{aligned}
$$

and $a \in C_{*}^{r} S_{1,1}^{m, \rho}, r \geq \rho>0$ iff

$$
\begin{array}{ll}
\left|\partial_{x}^{\alpha} \partial_{\eta^{\prime}}^{\beta} a\left(x, \eta^{\prime}\right)\right| \lesssim\left\langle\eta^{\prime}\right\rangle^{m-|\beta|} & \forall|\alpha|<\rho \\
\left\|\partial_{\eta^{\prime}}^{\beta} a\left(\cdot, \eta^{\prime}\right)\right\|_{C_{*}^{s}} \lesssim\left\langle\eta^{\prime}\right\rangle^{m-|\beta|+(s-\rho)^{+}} & \forall s \leq r
\end{array}
$$

If $\rho \leq 0$ and $r>0$ we denote in an abuse of notation $C_{*}^{r} S_{1,1}^{m, \rho}=C_{*}^{r} S_{1,1}^{m-\rho}$ in analoge to $S_{1,1}^{m, \rho}=S_{1,1}^{m-\rho}$.

At first we observe that it is sufficient to study $S_{1,1}^{m, \rho}$ and $C_{*}^{r} S_{1,1}^{m-\rho}$.

Proposition 2.7. A symbol $a \in C_{*}^{r} S_{1,1}^{m, \rho}$ can be split into

$$
a=a_{1}+a_{2}
$$

with $a_{1} \in S_{1,1}^{m, \rho}$ and $a_{2} \in C_{*}^{r} S_{1,1}^{m-\rho}$ with the additional estimates

$$
\left|\partial_{x}^{\alpha} \partial_{\eta^{\prime}}^{\beta} a_{2}\left(x, \eta^{\prime}\right)\right| \lesssim\left\langle\eta^{\prime}\right\rangle^{m-\rho+|\alpha|-|\beta|} \quad|\alpha|<\rho
$$

If the symbol a further satisfies the exotic behavior $\partial_{\xi^{\prime}}^{\beta} a \in C_{*}^{r+|\beta|} S^{m-|\beta|, \rho}$ for all $|\beta| \leq N$, then $\partial_{\xi^{\prime}}^{\beta} a_{2} \in C_{*}^{r+|\beta|} S_{1,1}^{m-\rho-|\beta|}$ for all $|\beta| \leq N$.

Proof. We can define the smoothing operator

$$
\chi\left(D_{x}, \xi\right)=\sum_{i \leq j} \psi_{i}\left(D_{x}\right) \psi_{j}\left(\eta^{\prime}\right)=\sum_{j} \varphi_{j+1}\left(D_{x}\right) \psi_{j}\left(\eta^{\prime}\right)
$$

with the principal support properties of $\chi$ illustrated in figure 1. Acting on the symbol, we obtain the decomposition $a=a_{1}+a_{2}$ with

$$
\begin{aligned}
& a_{1}\left(x, \eta^{\prime}\right)=\chi\left(D_{x}, \eta^{\prime}\right) a\left(x, \eta^{\prime}\right) \\
& a_{2}\left(x, \eta^{\prime}\right)=\left(1-\chi\left(D_{x}, \eta^{\prime}\right)\right) a\left(x, \eta^{\prime}\right)
\end{aligned}
$$

We check the definition of $S_{1,1}^{m, \rho}$ for $a_{1}$ and obtain for the partial derivatives 


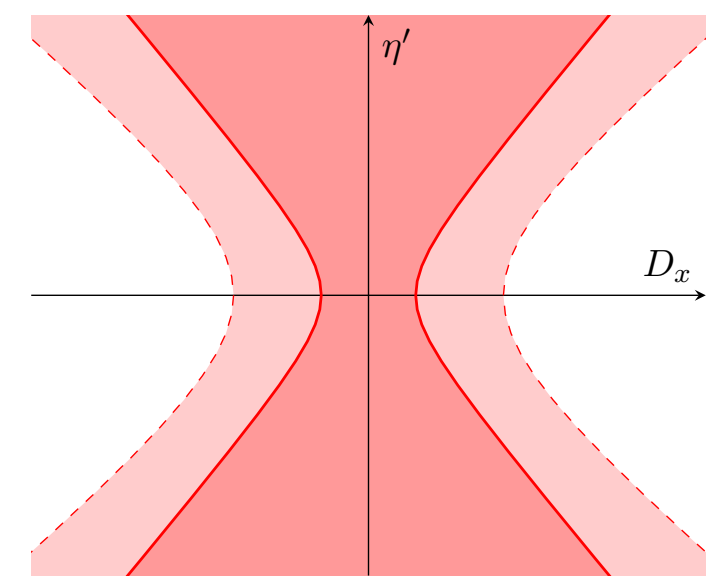

FiguRE 1. $\chi\left(D_{x}, \eta^{\prime}\right) \equiv 1$ within the inner, and supported within the outer hyperbolæ

$$
\left|\partial_{x}^{\alpha} \partial_{\eta^{\prime}}^{\beta} a_{1}\left(x, \eta^{\prime}\right)\right| \lesssim \sum_{i \leq j} 2^{i(|\alpha|-\rho)+j(m-|\beta|)} \psi_{j}\left(\eta^{\prime}\right) \lesssim\left\langle\eta^{\prime}\right\rangle^{m-|\beta|+(|\alpha|-\rho)_{\epsilon}^{+}} .
$$

And for the $C_{*}^{\rho}$ norm, we immediately obtain

$$
\left|\psi_{\mu}\left(D_{x}\right) \partial_{\eta^{\prime}}^{\beta} a_{1}\left(x, \eta^{\prime}\right)\right| \lesssim \sum_{\substack{i \leq j \\|\mu-i| \leq 1}} 2^{-i \rho+j(m-|\beta|)} \psi_{j}\left(\eta^{\prime}\right) \lesssim 2^{-\mu \rho}\left\langle\eta^{\prime}\right\rangle^{m-|\beta|} .
$$

Now considering $a_{2}$, we obtain for $|\alpha|<\rho$

$$
\left|\partial_{x}^{\alpha} \partial_{\eta^{\prime}}^{\beta} a_{2}\left(x, \eta^{\prime}\right)\right| \lesssim \sum_{i>j} 2^{i(|\alpha|-\rho)+j(m-|\beta|)} \psi_{j}\left(\eta^{\prime}\right) \lesssim\left\langle\eta^{\prime}\right\rangle^{m-\rho-|\beta|+|\alpha|}
$$

And for the $C_{*}^{s}$ norms, we immediately obtain for all $\rho \leq s \leq r$

$$
\left|\psi_{\mu}\left(D_{x}\right) \partial_{\eta^{\prime}}^{\beta} a_{2}\left(x, \eta^{\prime}\right)\right| \lesssim \sum_{\substack{i>j \\|\mu-i| \leq 1}} 2^{-i s+j(m-\rho-|\beta|+s)} \psi_{j}\left(\eta^{\prime}\right) \lesssim 2^{-\mu s}\left\langle\eta^{\prime}\right\rangle^{m-\rho-|\beta|+s}
$$

which yields the initial claim. If further $\partial_{\xi^{\prime}}^{\beta} a \in C_{*}^{r+|\beta|} S^{m-|\beta|, \rho}$ for all $|\beta| \leq N$, then

$$
\begin{aligned}
& \left|\psi_{\mu}\left(D_{x}\right) \partial_{\eta^{\prime}}^{\beta} a_{2}\left(x, \eta^{\prime}\right)\right| \lesssim \sum_{|\mu-i| \leq 1}\left|\psi_{i}\left(D_{x}\right) \partial_{\eta^{\prime}}^{\beta} \varphi_{i}\left(\eta^{\prime}\right) a\left(x, \eta^{\prime}\right)\right| \\
\lesssim & \sum_{\substack{|\mu-i| \leq 1 \\
\beta_{1}+\beta_{2}=\beta}} 2^{-i\left|\beta_{1}\right|}\left|\psi_{i}\left(D_{x}\right) \partial_{\eta^{\prime}}^{\beta_{2}} a\left(x, \eta^{\prime}\right)\right| \lesssim 2^{-\mu(r+|\beta|)}\left\langle\eta^{\prime}\right\rangle^{m-\rho+r}
\end{aligned}
$$

which concludes the second claim.

To analyze the distributions arising from these symbols, we need to give a decomposition in terms of shrinking cones around the $\xi^{\prime \prime}$-axis with respect to $\xi^{\prime}$. Unlike the projection of symbols of type $(1,0)$ to these regions, we will not have rapid decay in any cone away from the $\xi^{\prime}$-axis. We will only have decay in the $\langle\xi\rangle^{m}$ bounds as the angle of the cone around the $\xi^{\prime \prime}$-axis goes to 0 . So choose a smooth function $\tilde{\Psi}$ with

$$
\begin{aligned}
\tilde{\Psi}\left(t \xi^{\prime \prime}, t \xi^{\prime}\right) & =\tilde{\Psi}\left(\xi^{\prime \prime}, \xi^{\prime}\right) \forall\left|\xi^{\prime \prime}\right| \geq 2, t \geq 1 \\
\operatorname{supp} \tilde{\Psi} & \subseteq\left\{\left|\xi^{\prime \prime}\right| \geq \max \left(1,\left|\xi^{\prime}\right|\right)\right\} \\
\tilde{\Psi}\left(\xi^{\prime \prime}, \xi^{\prime}\right) & \equiv 1 \forall\left|\xi^{\prime \prime}\right| \geq 2 \max \left(1,\left|\xi^{\prime}\right|\right) .
\end{aligned}
$$


Then define a partition of unity via

$$
\begin{aligned}
\Psi_{s}\left(\xi^{\prime \prime}, \xi^{\prime}\right): & = \begin{cases}1-\tilde{\Psi}\left(\xi^{\prime \prime}, \xi^{\prime}\right) & s=-1 \\
\tilde{\Psi}\left(2^{-s} \xi^{\prime \prime}, \xi^{\prime}\right)-\tilde{\Psi}\left(2^{-s-1} \xi^{\prime \prime}, \xi^{\prime}\right) & s \geq 0\end{cases} \\
\operatorname{supp} \Psi_{s} & \subseteq \begin{cases}\left\{\left|\xi^{\prime \prime}\right| / 2 \leq \max \left(1,\left|\xi^{\prime}\right|\right)\right\} & s=-1 \\
\left\{\left|\xi^{\prime \prime}\right| / 4 \leq 2^{s} \max \left(1,\left|\xi^{\prime}\right|\right) \leq\left|\xi^{\prime \prime}\right|\right\} & s \geq 0 .\end{cases}
\end{aligned}
$$

With this and the Littlewood Paley decomposition $1=\sum \psi_{\nu}$, as described in A we

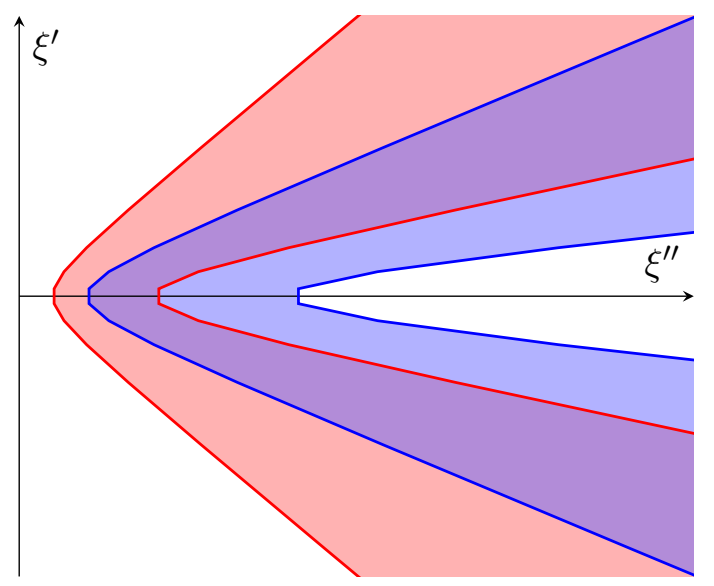

Figure 2. Support properties of $\Psi_{s}\left(\xi^{\prime \prime}, \xi^{\prime}\right)$ for $s=1$ and $s=2$ with overlap of their support

can give the natural Besov space embeddings of the distributions associated with these symbols.

Proposition 2.8. Let $m<0, r>0$ and $1<p \leq \infty$ with $m+r+k\left(1-\frac{1}{p}\right)>0$ and $a \in C_{*}^{r} S_{1,1}^{m}\left(\mathbb{R}^{n} \times \mathbb{R}^{k}\right)$. Then we have

$$
\begin{array}{r}
u(x)=\int e^{i x^{\prime} \eta^{\prime}} a\left(x, \eta^{\prime}\right) d \eta^{\prime} \in \text { unif } B_{p, \infty}^{-m-k(1-1 / p)}\left(\mathbb{R}^{n}\right) \\
a \in C_{*}^{r} S_{1,1}^{m}\left(\mathbb{R}^{n-k} \times \mathbb{R}^{k}\right), \forall r>0 \Rightarrow \operatorname{sing} \operatorname{supp}(u) \subseteq \mathbb{R}^{n-k} \times\{0\}
\end{array}
$$

Proof. Without loss of generality let $a$ have compact support in $x^{\prime \prime}$. First we need to subdivide the symbol into regions of different behavior in the Fourier image.

$$
\hat{a}\left(\xi^{\prime \prime}, \zeta^{\prime}, \eta^{\prime}\right)=\sum_{s, \mu=-1}^{\infty} \Psi_{s}\left(\xi^{\prime \prime}, \zeta^{\prime}+\eta^{\prime}\right) \psi_{\mu}\left(\eta^{\prime}\right) \hat{a}\left(\xi^{\prime \prime}, \zeta^{\prime}, \eta^{\prime}\right)
$$

The components behave differently if $s=-1$ or not giving us two cases. Aiming at Besov norms we are interested if $\xi=\left(\xi^{\prime \prime}, \zeta^{\prime}+\eta^{\prime}\right)$ is in the support of $\psi_{\nu}(\xi)$, i.e. a given annulus $2^{\nu-1} \leq|\xi| \leq 2^{\nu+1}$ for some $\nu \geq 0$ or $|\xi| \leq 1$ for $\nu=-1$. If $s=-1$ we have in the support of $\Psi_{s}$ that

$$
2 \max \left(1,\left|\xi^{\prime}\right|\right) \geq\left|\xi^{\prime \prime}\right| \quad \Rightarrow \quad\langle\xi\rangle \leq 2\left\langle\xi^{\prime}\right\rangle \quad \Rightarrow \quad\langle\xi\rangle \sim\left\langle\xi^{\prime}\right\rangle
$$

Vice versa if $s \geq 0$ we have in the support of $\Psi_{s}$ that

$$
\left|\xi^{\prime \prime}\right| \geq 2^{s} \max \left(1,\left|\xi^{\prime}\right|\right) \quad \Rightarrow \quad\langle\xi\rangle \leq 2\left\langle\xi^{\prime \prime}\right\rangle \quad \Rightarrow \quad\langle\xi\rangle \sim\left\langle\xi^{\prime \prime}\right\rangle
$$

Here we further have $\left|\xi^{\prime \prime}\right| \leq 2^{s+2} \max \left(1,\left|\xi^{\prime}\right|\right)$ implying $\left\langle\xi^{\prime \prime}\right\rangle \sim 2^{s}\left\langle\xi^{\prime}\right\rangle$, also observe that $s \leq \nu+1$. 
Note that in general $\left\langle\xi^{\prime}\right\rangle \sim 2^{\nu-s}$. We summarize these cutoffs in a partition function

$$
\Gamma_{\nu, s, \mu}=\psi_{\nu}(\xi) \Psi_{s}(\xi) \psi_{\mu}\left(\eta^{\prime}\right)
$$

To compute the Besov norm, $\nu$ is fixed and we sum over $s, \mu \geq-1$ with the restriction $s \leq \nu+1$. Using integration by parts for these cutoff functions, we obtain the following estimate on the Fourier inverse

$$
\begin{aligned}
& \left|\partial_{\xi^{\prime \prime}}^{\alpha} \partial_{\xi^{\prime}}^{\beta} \partial_{\eta^{\prime}}^{\gamma} \Gamma_{\nu, s, N, \mu}\right| \lesssim 2^{-\nu|\alpha|-(\nu-s)|\beta|-\mu|\gamma|} \\
& \left|\partial_{\eta^{\prime}}^{\gamma} \check{\Gamma}_{\nu, s, N, \mu}\left(y, \eta^{\prime}\right)\right| \lesssim 2^{\nu n-s k-\mu|\gamma|}\left\langle 2^{\nu} y^{\prime \prime}\right\rangle^{-M}\left\langle 2^{\nu-s} y^{\prime}\right\rangle^{-M}
\end{aligned}
$$

Note that $2^{\nu n-s k}$ is an estimate for the volume of the support in $\xi$. Now we investigate the corresponding function to the cutoff, first observing its Fourier transform

$$
\hat{h}_{\nu, s, \mu}(\xi)=\int \hat{a}\left(\xi^{\prime \prime}, \xi^{\prime}-\eta^{\prime}, \eta^{\prime}\right) \Gamma_{\nu, s, \mu}\left(\xi, \eta^{\prime}\right) \mathrm{d} \eta^{\prime}
$$

Now we probe the two cases $\mu \leq \nu-3$ and $\mu>\nu-3$ separately.

1. Let $\mu \leq \nu-3$ and define $\zeta=\left(\xi^{\prime \prime}, \xi^{\prime}-\eta^{\prime}\right)$. Then for $\left(\xi, \eta^{\prime}\right) \in \operatorname{supp} \Gamma_{\nu, s, \mu}$ using $\left|\eta^{\prime}\right| \leq 2^{\nu-2}$ we have

$$
\begin{aligned}
& |\zeta| \leq|\xi|+\left|\eta^{\prime}\right| \leq 2^{\nu+1}+2^{\nu-2} \leq 2^{\nu+2} \\
& |\zeta| \geq|\xi|-\left|\eta^{\prime}\right| \geq 2^{\nu-1}-2^{\nu-2} \geq 2^{\nu-2}
\end{aligned}
$$

on the support of $\Gamma_{\nu, s, \mu}$. We have $\sum_{\ell=-3}^{3} \psi_{\nu+\ell}(\zeta)=1$ on the support of $\Gamma_{\nu, s, \mu}$ and we obtain

$$
\begin{aligned}
& \hat{h}_{\nu, s, \mu}(\xi)=\int \sum_{\ell=-3}^{3} \psi_{\nu+\ell}\left(\xi^{\prime \prime}, \xi^{\prime}-\eta^{\prime}\right) \hat{a}\left(\xi^{\prime \prime}, \xi^{\prime}-\eta^{\prime}, \eta^{\prime}\right) \Gamma_{\nu, s, \mu}\left(\xi, \eta^{\prime}\right) \mathrm{d} \eta^{\prime} \\
& h_{\nu, s, \mu}(x)=\int e^{i \eta^{\prime}\left(x^{\prime}-y^{\prime}\right)} \sum_{\ell=-3}^{3} \psi_{\nu+\ell}\left(D_{x}\right) a\left(x-y, \eta^{\prime}\right) \check{\Gamma}_{\nu, s, N, \mu}\left(y, \eta^{\prime}\right) \mathrm{d} y \mathrm{~d} \eta^{\prime} .
\end{aligned}
$$

Using the symbol property $\left\|\partial_{\eta^{\prime}}^{\beta} a\left(\cdot, \eta^{\prime}\right)\right\|_{C_{*}^{r}} \lesssim\left\langle\eta^{\prime}\right\rangle^{m-|\beta|+r}$ we utilize proposition A.7 with $d=d\left(x^{\prime \prime}\right)=\operatorname{dist}\left(x^{\prime \prime}, \operatorname{supp} a\left(\cdot, x^{\prime}, \eta^{\prime}\right)+B_{\epsilon}\right)$ and we obtain as an estimate through integration by parts with respect to $\eta^{\prime}$

$$
\begin{aligned}
& \left|h_{\nu, s, \mu}(x)\right| \lesssim \\
& 2^{\nu n-s k-\nu r+\mu(m+r+k)}\left\langle 2^{\nu} d\right\rangle^{-M} \int\left\langle 2^{\nu} y^{\prime \prime}\right\rangle^{-M}\left\langle 2^{\nu-s} y^{\prime}\right\rangle^{-M}\left\langle 2^{\mu}\left(x^{\prime}-y^{\prime}\right)\right\rangle^{-M} \mathrm{~d} y
\end{aligned}
$$

2. If $\nu-3<\mu$ we instead obtain

$$
\begin{aligned}
h_{\nu, s, \mu}(x) & =\int e^{i \eta^{\prime}\left(x^{\prime}-y^{\prime}\right)} a\left(x-y, \eta^{\prime}\right) \check{\Gamma}_{\nu, s, \mu}\left(y, \eta^{\prime}\right) \mathrm{d} y \mathrm{~d} \eta^{\prime} \\
\left|h_{\nu, s, \mu}(x)\right| & \lesssim 2^{\nu n-s k+\mu(m+k)} \int_{\left|y^{\prime \prime}\right| \geq d}\left\langle 2^{\nu} y^{\prime \prime}\right\rangle^{-M}\left\langle 2^{\nu-s} y^{\prime}\right\rangle^{-M}\left\langle 2^{\mu}\left(x^{\prime}-y^{\prime}\right)\right\rangle^{-M} \mathrm{~d} y
\end{aligned}
$$

We obtain as $L^{p}$ estimates from scaling and convolution estimates

$$
\left\|h_{\nu, s, \mu}\right\|_{L^{p}} \lesssim \begin{cases}2^{-\nu r+\mu(m+r+k / q)} & \mu \leq \nu-s \\ 2^{(\nu-s) k / q-\nu r+\mu(m+r)} & \nu-s<\mu \leq \nu-3 \\ 2^{(\nu-s) k / q+\mu m} & \nu-3<\mu .\end{cases}
$$


Now we can estimate the Besov norm by estimating

$$
\begin{aligned}
\sum_{s=-1}^{\nu+1} \sum_{\mu=-1}^{\infty}\left\|h_{\nu, s, \mu}\right\|_{L^{p}} & =\left(\sum_{s=-1}^{\nu+1} \sum_{\mu=-1}^{\nu-s}+\sum_{s=-1}^{\nu+1} \sum_{\mu=\nu-s+1}^{\nu-3}+\sum_{s=-1}^{\nu+1} \sum_{\mu=\nu-2}^{\infty}\right)\left\|h_{\nu, s, \mu}\right\|_{L^{p}} \\
\sum_{s=-1}^{\nu+1} \sum_{\mu=-1}^{\nu-s}\left\|h_{\nu, s, \mu}\right\|_{L^{p}} & \lesssim \sum_{s=-1}^{\nu+1} \sum_{\mu=-1}^{\nu-s} 2^{-\nu r+\mu(m+r+k / q)} \\
& \lesssim \sum_{s=-1}^{\nu+1} 2^{\nu(m+k / q)-s(m+r+k / q)} \lesssim 2^{\nu(m+k / q)} \\
\sum_{s=-1}^{\nu+1} \sum_{\mu=\nu-s+1}^{\nu-3}\left\|h_{\nu, s, \mu}\right\|_{L^{p}} & \lesssim \sum_{s=-1}^{\nu+1} \sum_{\mu=\nu-s+1}^{\nu-3} 2^{(\nu-s) k / q-\nu r+\mu(m+r)} \\
& \lesssim \sum_{s=-1}^{\nu+1} \sum_{\mu=\nu-s+1}^{\nu} 2^{(\nu-s) k / q-\nu r+\nu(m+r)+s(m+r)^{-}} \\
& \lesssim \sum_{s=-1}^{\nu+1} s 2^{\nu(m+k / q)+s\left((m+r)^{-}-k / q\right)} \lesssim 2^{\nu(m+k / q)} \\
\sum_{s=-1}^{\nu+1} \sum_{\mu=\nu-2}^{\infty}\left\|h_{\nu, s, \mu}\right\|_{L^{p}} & \lesssim \sum_{s=-1}^{\nu+1} \sum_{\mu=\nu-2}^{\infty} 2^{(\nu-s) k / q+\mu m} \lesssim \sum_{s=-1}^{\nu+1} 2^{\nu(m+k / q)-s k / q} \\
& \lesssim 2^{\nu(m+k / q)} .
\end{aligned}
$$

The second assertion is obvious, as multiplication by $x^{\prime}$ is equivalent to differentiation with respect to $\eta^{\prime}$, corresponding to a regularity improvement, hence $u$ is smooth away from $x^{\prime}=0$.

Remark 2.9. Combining the Propositions 2.7 and 2.8, we can always reduce a full symbol $a$ to having spectral support around a unit cone around the $\eta^{\prime}$-axis or plane. Then given $N \in \mathbb{N}_{0}$ and $1<p \leq \infty$, we can define the seminorm $\|\cdot\|_{N, p}$ for such a decomposition $u=u_{C}+u_{G} \in I_{1,1 ; \ell}^{m, \rho}$

$$
\left\|\left(u_{C}, u_{G}\right)\right\|_{N, p}=\sum_{|\beta| \leq N} \sup _{\eta^{\prime}} \frac{\left\|\partial_{\eta^{\prime}}^{\beta} a\left(\cdot, \eta^{\prime}\right)\right\|_{C_{*}^{\rho}}}{\left\langle\eta^{\prime}\right\rangle^{m-|\beta|}}+\sum_{\left|\alpha^{\prime}\right| \leq \min (N, \ell)}\left\|\left(x^{\prime}\right)^{\alpha^{\prime}} u_{G}\right\|_{\text {unif } B_{p, \infty}^{-m+\rho-k(1-1 / p)}} .
$$

And then we can define on $I_{1,1 ; \ell}^{m, \rho}$ the seminorms

$$
\|u\|_{N, p}=\inf _{u=u_{C}+u_{G}}\left\{\left\|\left(u_{C}, u_{G}\right)\right\|_{N, p}+\left\|\left(u_{C}, u_{G}\right)\right\|_{N, \infty}\right\} .
$$

1.3. Symbol Reduction and Improved Smoothness. The shortcoming of Proposition 2.8 become clear if compared to the corresponding result for conormal distributions of type $(1,0)$ type. Here $(1)$ is still optimal but (2) can be improved to

$$
u \in I_{1,0}^{m}\left(\mathbb{R}^{n}, \mathbb{R}^{n-k}\right) \Rightarrow \mathrm{WF}(u) \subseteq N^{*} \mathbb{R}^{n-k} .
$$

So Proposition 2.8 alone tells us almost nothing about the microlocal structure of conormal distributions of type $(1,1)$. Thus improved regularity needs to be used to learn about the microlocal properties of our distributions. In fact, we can immediately obtain the following result.

Corollary 2.10. For all $u \in I_{1,1}^{m, \rho}\left(\mathbb{R}^{n}, \mathbb{R}^{n-k}\right)$ we have

$$
u \in G^{m-\rho}(x, \xi) \quad \forall(x, \xi) \in T^{*} \mathbb{R}^{n} \backslash N^{*} \mathbb{R}^{n-k} .
$$


Proof. For all $d \in \mathbb{N}$ define the generalization of $\chi(D)$ form Proposition 2.7

$$
\chi_{d}(D)=\sum_{i \leq j-d} \psi_{i}\left(D_{x^{\prime \prime}}\right) \psi_{j}\left(D_{x^{\prime}}\right)=\sum_{j} \varphi_{j+1}\left(D_{x^{\prime \prime}}\right) \psi_{j-d}\left(D_{x^{\prime}}\right) .
$$

By Proposition 2.7 and 2.8, we have

$$
\left(1-\chi_{d}(D)\right) u \in G^{m-\rho}
$$

which yields the claim, as $u \in G^{m-\rho}\left(x^{\prime \prime}, x^{\prime}\right)$ for all $x^{\prime} \neq 0$ by Proposition 2.8 .

In most computations we can more or less disregard the symbolic structure of a distribution without improved regularity. This is the precise reason for introducing $G^{m, \ell}$ as a remainder space. However, in the following technical construction, we will learn that this is only true for directional derivatives in $x^{\prime \prime}$-coordinates. To see this effect, we need to introduce a generalization of the already defined symbols. These do appear in computations.

Definition 2.11. A symbol is called a symbol with unidirectional improved smoothness $\rho^{\prime \prime}>0$, if it suffices

$$
\begin{aligned}
\left|\psi_{\mu}\left(D_{x^{\prime \prime}}\right) \partial_{x^{\prime}}^{\alpha^{\prime}} \partial_{\eta^{\prime}}^{\beta} a\left(x, \eta^{\prime}\right)\right| & \lesssim 2^{-\mu \rho^{\prime \prime}}\left\langle\eta^{\prime}\right\rangle^{m-|\beta|+\left|\alpha^{\prime}\right|} \\
\left|\partial_{x^{\prime}}^{\alpha^{\prime}} \partial_{x^{\prime \prime}}^{\alpha^{\prime \prime}} \partial_{\eta^{\prime}}^{\beta} a\left(x, \eta^{\prime}\right)\right| & \lesssim\left\langle\eta^{\prime}\right\rangle^{m-|\beta|+\left|\alpha^{\prime}\right|+\left(\left|\alpha^{\prime \prime}\right|-\rho^{\prime \prime}\right)^{+}} \quad \forall\left|\alpha^{\prime \prime}\right| \neq \rho^{\prime \prime}
\end{aligned}
$$

write $a \in S_{1,1}^{m, \rho^{\prime \prime}, 0}\left(\mathbb{R}^{n} \times \mathbb{R}^{k}\right)$. For $\rho^{\prime \prime}, \rho^{\prime}>0$ a symbol is called a symbol with anisotropic improved smoothness $\left(\rho^{\prime}, \rho^{\prime \prime}\right)$ if

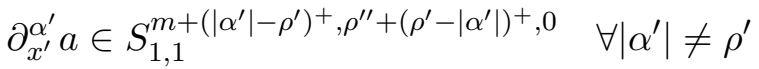

$$
\begin{aligned}
& \partial_{x^{\prime}}^{\alpha^{\prime}} a \in S_{1,1}^{m+\epsilon, \rho^{\prime \prime}+\epsilon, 0} \quad \forall\left|\alpha^{\prime}\right|=\rho^{\prime}, \epsilon>0
\end{aligned}
$$

write $a \in S_{1,1}^{m, \rho^{\prime \prime}, \rho^{\prime}}$. We define $S_{1,1}^{m, 0, \rho^{\prime}}$ to be $S_{1,1}^{m, \rho^{\prime}}$.

Proposition 2.12. For a symbol a with anisotropic improved smoothness $\left(\rho^{\prime}, \rho^{\prime \prime}\right)$ and order $m$ with $m-\rho^{\prime}+k<0$, there is a symbol $b_{1} \in S_{1,1}^{m, \rho^{\prime \prime}+\rho^{\prime}}\left(\mathbb{R}^{n-k} \times \mathbb{R}^{k}\right)$ and a remainder function $b_{2} \in G^{m-\rho^{\prime \prime}-\rho^{\prime}, \infty}$ such that

$$
\int e^{i x^{\prime} \eta^{\prime}} a\left(x, \eta^{\prime}\right) d \eta^{\prime}=\int e^{i x^{\prime} \eta^{\prime}} b_{1}\left(x^{\prime \prime}, \eta^{\prime}\right) d \eta^{\prime}+b_{2}(x) \in I_{1,1 ; \infty}^{m, \rho} .
$$

And furthermore we have the expansion

$$
\begin{aligned}
& b_{1}\left(x^{\prime \prime}, \eta^{\prime}\right)-\sum_{\left|\alpha^{\prime}\right|<N} \partial_{x^{\prime}}^{\alpha}\left(i \partial_{\eta^{\prime}}\right)^{\alpha} a\left(x^{\prime \prime}, 0, \eta^{\prime}\right) / \alpha ! \in S_{1,1}^{m-\max \left(N, \rho^{\prime}\right), \rho^{\prime \prime}+\left(\rho^{\prime}-N\right)^{+}} \forall N \neq \rho^{\prime} \\
& b_{1}\left(x^{\prime \prime}, \eta^{\prime}\right)-\sum_{\left|\alpha^{\prime}\right|<N} \partial_{x^{\prime}}^{\alpha}\left(i \partial_{\eta^{\prime}}\right)^{\alpha} a\left(x^{\prime \prime}, 0, \eta^{\prime}\right) / \alpha ! \in S_{1,1}^{m+\epsilon, \rho^{\prime \prime}+\epsilon} \quad N=\rho^{\prime}, \forall \epsilon>0 .
\end{aligned}
$$

Proof. We need to find a symbol representing the remainder

$$
\int e^{i x^{\prime} \xi^{\prime}} r\left(x^{\prime \prime}, \xi^{\prime}\right) \mathrm{d} \xi^{\prime}=\int e^{i x^{\prime} \xi^{\prime}}\left[a\left(x, \xi^{\prime}\right)-\sum_{|\alpha|<N} \partial_{x^{\prime}}^{\alpha}\left(i \partial_{\xi^{\prime}}\right)^{\alpha} a\left(x, \xi^{\prime}\right)_{\left.\right|_{x^{\prime}=0}} / \alpha !\right] \mathrm{d} \xi
$$

and obtain sufficient estimates on it. We can restrict to the case $N>\rho^{\prime}$, as the estimates on the approximation terms imply the other cases. Therefore we decompose our symbol into

$$
a_{i, j}\left(x, \eta^{\prime}\right)=\psi_{i}\left(D_{x^{\prime \prime}}\right) \psi_{j}\left(\eta^{\prime}\right) a\left(x, \eta^{\prime}\right)
$$


Then we have with $M>0$ some big constant

$$
\left|\partial_{x^{\prime}}^{\alpha^{\prime}} \partial_{x^{\prime \prime}}^{\alpha^{\prime \prime}} \partial_{\eta^{\prime}}^{\beta} a_{i, j}\right| \lesssim 2^{j\left[m+\left(\left|\alpha^{\prime}\right|-\rho^{\prime}\right)^{+}-|\beta|\right]} 2^{i\left(\left|\alpha^{\prime \prime}\right|-\rho^{\prime \prime}-\left(\rho^{\prime}-\left|\alpha^{\prime}\right|\right)^{+}\right)} 2^{-(i-j)^{+} M} .
$$

Now we define rescaled symbols

$$
A_{i, j}\left(x, \eta^{\prime}\right)=2^{-j m+i \rho^{\prime \prime}+(i-j)^{+} M} a_{i, j}\left(2^{-i} x^{\prime \prime}, 2^{-j} x^{\prime}, 2^{j} \eta^{\prime}\right) .
$$

Now with standard theory of conormal distributions of type $(1,0)$ - see for instance the usage in Lemma 9.6.1 in $[\mathbf{H o ̈ 0 3 b}$, the proof of which is almost parallel - as the rescaled symbols are uniformly bounded in $S_{1,0}^{-M}$, we have

$$
\begin{aligned}
& B_{i, j}\left(x^{\prime \prime}, \xi^{\prime}\right)=\int e^{i x^{\prime}\left(\eta^{\prime}-\xi^{\prime}\right)} A_{i, j}\left(x, \eta^{\prime}\right) \mathrm{d} x^{\prime} \mathrm{d} \eta^{\prime} \in S_{1,0}^{-M} \\
& R_{i, j}\left(x^{\prime \prime}, \xi^{\prime}\right)=B_{i, j}\left(x^{\prime \prime}, \xi^{\prime}\right)-\sum_{\left|\alpha^{\prime}\right|<N} \partial_{x^{\prime}}^{\alpha^{\prime}}\left(i \partial_{\xi^{\prime}}\right)^{\alpha^{\prime}} A_{i, j}\left(x, \xi^{\prime}\right)_{\left.\right|_{x^{\prime}=0}} / \alpha ! \in S_{1,0}^{-M-N}
\end{aligned}
$$

with seminorms of $R_{i, j}$ bounded by seminorms of $\partial_{x^{\prime}}^{\alpha^{\prime}} \partial_{\xi^{\prime}}^{\alpha^{\prime}} A_{i, j}\left(x, \xi^{\prime}\right)$ with $\left|\alpha^{\prime}\right|=N$, which are bounded by $2^{-j \rho^{\prime}}$. So by scaling back, we obtain

$$
\begin{aligned}
r_{i, j}\left(x^{\prime \prime}, \xi^{\prime}\right) & =2^{j m-i \rho^{\prime \prime}-(i-j)^{+} M} R_{i, j}\left(2^{i} x^{\prime \prime}, 2^{-j} \xi^{\prime}\right) \\
\left|\partial_{\xi^{\prime}}^{\beta} r_{i, j}\left(x^{\prime \prime}, \xi^{\prime}\right)\right| & \lesssim 2^{j\left(m-\rho^{\prime}-|\beta|\right)-i \rho^{\prime \prime}-(i-j)^{+} M}\left\langle 2^{-j} \xi^{\prime}\right\rangle^{-(M+|\beta|)}
\end{aligned}
$$

with spectral support of $r_{i, j}$ within the support of $\psi_{i}\left(\xi^{\prime \prime}\right)$. Formally, we have

$$
\sum_{i, j} \int e^{i x^{\prime} \xi^{\prime}} r_{i, j}\left(x^{\prime \prime}, \xi^{\prime}\right) \mathrm{d} \xi^{\prime}=\int e^{i x^{\prime} \xi^{\prime}}\left[a\left(x, \xi^{\prime}\right)-\sum_{|\alpha|<N} \partial_{x^{\prime}}^{\alpha}\left(i \partial_{\xi^{\prime}}\right)^{\alpha} a\left(x, \xi^{\prime}\right)_{\left.\right|_{x^{\prime}=0}} / \alpha !\right] \mathrm{d} \xi .
$$

So it remains to find the symbol class of $r=\sum r_{i, j}$. So let $2^{\nu} \geq\left\langle\xi^{\prime}\right\rangle$, then we can estimate

$$
\begin{aligned}
\left|\psi_{\nu}\left(D_{x^{\prime \prime}}\right) r\left(x^{\prime \prime}, \xi^{\prime}\right)\right| & \lesssim 2^{-\nu \rho^{\prime \prime}}\left(\sum_{j \leq \nu} 2^{j\left(m-\rho^{\prime}-|\beta|+M\right)-\nu M}+\sum_{j \geq \nu} 2^{j\left(m-\rho^{\prime}-|\beta|\right)}\right) \\
& \lesssim 2^{\nu\left(m-\rho^{\prime}-\rho^{\prime \prime}\right)}
\end{aligned}
$$

and for $2^{\nu} \leq\left\langle\xi^{\prime}\right\rangle$, we obtain

$$
\begin{aligned}
\left|\psi_{\nu}\left(D_{x^{\prime \prime}}\right) r\left(x^{\prime \prime}, \xi^{\prime}\right)\right| & \lesssim 2^{-\nu \rho^{\prime \prime}}\left(\sum_{2^{j} \leq\left\langle\xi^{\prime}\right\rangle} 2^{j\left(m-\rho^{\prime}-|\beta|+M\right)}\left\langle\xi^{\prime}\right\rangle^{-M}+\sum_{2^{j} \geq\left\langle\xi^{\prime}\right\rangle} 2^{j\left(m-\rho^{\prime}-|\beta|\right)}\right) \\
& \lesssim 2^{-\nu \rho^{\prime \prime}}\left\langle\xi^{\prime}\right\rangle^{m-\rho^{\prime}-|\beta|} .
\end{aligned}
$$

This implies $\partial_{\xi^{\prime}}^{\beta} r\left(x^{\prime \prime}, \xi^{\prime}\right) \in C_{*}^{-m+\rho^{\prime}+\rho^{\prime \prime}+|\beta|} S^{m-\rho^{\prime}-|\beta|, \rho^{\prime \prime}}$ and with the Propositions 2.7 and 2.8 , we obtain the claim.

\section{Pseudodifferential operators of type $(1,1)$}

Pseudodifferential operators of type $(1,1)$ appear in calculations for nonlinear superposition, paraproducts and a bunch of other techniques. We will apply theses methods in the later study and therefore we need to give the mapping properties and the asymptotic expansion of these types of operators. Note that the approach used in Lemma 2.13 - splitting the domain of integration and taking partial derivatives respectively - is going to be reused in other proofs, where the arguments are not going to be as rigid as here. The idea for proofs like this is similar to the proof of [Hö03b, Lemma 9.3.2]. 
Lemma 2.13. Let $p(x, \xi) \in S_{1,1}^{m}\left(\mathbb{R}^{n} \times \mathbb{R}^{n}\right)$ then for all $s-m>0$ we have

$$
p(x, D): B_{p, \infty}^{s}\left(\mathbb{R}^{n}\right) \rightarrow B_{p, \infty}^{s-m}\left(\mathbb{R}^{n}\right)
$$

If $p\left(x, \xi^{\prime \prime}\right) \in S_{1,1}^{m}\left(\mathbb{R}^{n} \times \mathbb{R}^{n-k}\right)$ we have for all $s-m>0$

$$
p\left(x, D_{x^{\prime \prime}}\right): C_{*}^{s}\left(\mathbb{R}^{n-k}\right) \rightarrow C_{*}^{s-m}\left(\mathbb{R}^{n}\right)
$$

Proof. We consider the kernel of the operator $\psi_{\mu}(D) p\left(x, D_{x^{\prime \prime}}\right) \psi_{\nu}\left(D_{x^{\prime \prime}}\right)$

$$
K_{\mu, \nu}\left(x, y^{\prime \prime}\right)=\int e^{i\left(z^{\prime \prime}-y^{\prime \prime}\right) \xi^{\prime \prime}+i(x-z) \eta} \psi_{\mu}(\eta) p\left(z, \xi^{\prime \prime}\right) \psi_{\nu}\left(\xi^{\prime \prime}\right) \mathrm{d} z \mathrm{~d} \eta \mathrm{d} \xi^{\prime \prime}
$$

First choose a smooth partition of unity on the unit sphere $1=\sum_{\ell=1}^{n} \chi_{\ell}\left(\frac{\xi}{|\xi|}\right)$ such that for some $\varphi \in C_{c}^{\infty}$ with $\varphi \equiv 1$ in a neighborhood of 0 we have

$$
\chi_{\ell}(\xi)=(1-\varphi(\xi)) \chi_{\ell}\left(\frac{\xi}{|\xi|}\right) \quad \operatorname{supp} \chi_{\ell}(\cdot) \subseteq\left\{\left|\xi_{\ell}\right| \gtrsim 1\right\} .
$$

Then for $\mu>0$ cut $\psi_{\mu}(\eta)$ into the respective sectors around the axis, meaning $\psi_{\mu}(\eta)=\sum_{\ell} \psi_{\mu, \ell}(\eta)=\sum_{\ell} \psi_{\mu}(\eta) \chi_{\ell}(\eta)$. If $\mu \geq \nu+n_{0}$ we use integration by parts on $z$ of the respective axis. As $\left|\xi^{\prime \prime}-\eta^{\prime \prime}\right| \gtrsim 2^{\mu}$ on the respective sectors given $n_{0}$ large enough, we obtain

$$
\begin{array}{r}
K_{\mu, \nu}\left(x, y^{\prime \prime}\right)=\sum_{\ell} \int e^{i\left(z^{\prime \prime}-y^{\prime \prime}\right) \xi^{\prime \prime}+i(x-z) \eta}\left(\frac{i}{\eta^{\prime \prime}-\xi^{\prime \prime}}\right)^{\alpha_{\ell}^{\prime \prime}}\left(\frac{i}{\eta^{\prime}}\right)^{\alpha_{\ell}^{\prime}} \\
\psi_{\mu, \ell}(\eta) \partial_{z}^{\alpha} p\left(z, \xi^{\prime \prime}\right) \psi_{\nu}\left(\xi^{\prime \prime}\right) \mathrm{d} z \mathrm{~d} \eta \mathrm{d} \xi^{\prime \prime}
\end{array}
$$

Now splitting the integral into regions $A$, where $|x-z|<\epsilon$ or $\left|x_{j}-z_{j}\right| \geq \epsilon / \sqrt{n}$ combined with $\left|z^{\prime \prime}-y^{\prime \prime}\right|<\delta$ or $\left|z_{j}^{\prime \prime}-y_{j}^{\prime \prime}\right| \geq \delta / \sqrt{n}$ holds, we obtain using partial integration again

$$
\begin{aligned}
\sum_{A, \ell} \int_{A} e^{i\left(z^{\prime \prime}-y^{\prime \prime}\right) \xi^{\prime \prime}+i(x-z) \eta[}\left[\partial_{\xi^{\prime \prime}} \frac{i}{z^{\prime \prime}-y^{\prime \prime}}\right]^{\beta_{A}^{\prime \prime}} & {\left[\partial_{\eta} \frac{i}{x-z}\right]^{\gamma_{A}}\left(\frac{i}{\eta^{\prime \prime}-\xi^{\prime \prime}}\right)^{\alpha_{\ell}^{\prime \prime}}\left(\frac{i}{\eta^{\prime}}\right)^{\alpha_{\ell}^{\prime}} } \\
& \psi_{\mu, \ell}(\eta) \partial_{z}^{\alpha} p\left(z, \xi^{\prime \prime}\right) \psi_{\nu}\left(\xi^{\prime \prime}\right) \mathrm{d} z \mathrm{~d} \eta \mathrm{d} \xi^{\prime \prime}
\end{aligned}
$$

Taking estimates, we obtain

$$
\begin{array}{r}
\left|K_{\mu, \nu}\left(x, y^{\prime \prime}\right)\right| \lesssim \int\left\langle 2^{\nu}\left(z^{\prime \prime}-y^{\prime \prime}\right)\right\rangle^{-N}\left\langle 2^{\mu}(x-z)\right\rangle^{N} 2^{\nu(m+(n-k)+L)+\mu(n-L)} \mathrm{d} z \\
\lesssim 2^{\nu m-L(\mu-\nu)^{+}} 2^{\min (\nu, \mu)(n-k)}\left\langle 2^{\min (\nu, \mu)}\left(y^{\prime \prime}-x^{\prime \prime}\right)\right\rangle^{-N}
\end{array}
$$

Which yields as an estimate if $u \in B_{p, \infty}^{s}\left(\mathbb{R}^{n-k}\right)$ and $m-s<0$ choosing $L+m-s>0$ :

$$
\begin{aligned}
& \left\|\psi_{\mu}(D) p\left(x, D_{x^{\prime \prime}}\right) u\right\|_{L_{x^{\prime \prime}}^{\infty}\left(\mathbb{R}^{n-k} ; L_{x^{\prime}}^{p}\left(\mathbb{R}^{k}\right)\right)} \\
& \lesssim \sum_{\nu} 2^{\nu m-L(\nu-\mu)^{+}}\left\|\int 2^{\min (\nu, \mu)(n-k)}\left\langle 2^{\min (\nu, \mu)}\left(x^{\prime \prime}-y^{\prime \prime}\right)\right\rangle^{-N} \tilde{\psi}_{\nu}\left(D_{x^{\prime \prime}}\right) u\left(y^{\prime \prime}\right) \mathrm{d} y^{\prime \prime}\right\|_{L^{p}} \\
& \lesssim \sum_{\nu} 2^{\nu m-L(\nu-\mu)^{+}} 2^{-\nu s}=\sum_{\nu=-1}^{\mu} 2^{\nu(m-s+L)} 2^{-\mu L}+\sum_{\nu=\mu+1}^{\infty} 2^{\nu(m-s)} \lesssim 2^{\mu(m-s)}
\end{aligned}
$$

Which finishes the proof as the first assertion follows from $k=0$ and the second from $p=\infty$. 
Having shown the regularity of the operators on the remainder spaces, it is an easy rescaling argument to show the natural asymptotic expansion formula. The only thing one needs to take care of are spectral support properties of the respective operator and distributional symbols. By a simple reduction argument we can obtain a situation with well behaved support properties.

To ease readability for symbolic computations introduce the notation

$$
(p \# a)\left(x^{\prime \prime}, \xi^{\prime}\right)=\int e^{-i \eta^{\prime \prime}\left(y^{\prime \prime}-x^{\prime \prime}\right)+i y^{\prime}\left(\eta^{\prime}-\xi^{\prime}\right)} p\left(x^{\prime \prime}, y^{\prime}, \eta\right) a\left(y^{\prime \prime}, \eta^{\prime}\right) \mathrm{d} \eta \mathrm{d} y
$$

which is just the partial Fourier transform of $p(x, D) \int e^{i x^{\prime} \eta^{\prime}} a\left(x^{\prime \prime}, \eta^{\prime}\right) \mathrm{d} \eta^{\prime}$.

Theorem 2.14. Let $p(x, \xi) \in S_{1,1}^{m_{1}, \rho_{1}}\left(\mathbb{R}^{n} \times \mathbb{R}^{n}\right)$ and $u \in I_{1,1}^{m_{2}, \rho_{2}}\left(\mathbb{R}^{n}, \mathbb{R}^{n-k}\right)$ with full symbol $a\left(x^{\prime \prime}, \xi^{\prime}\right) \in S_{1,1}^{m_{2}, \rho_{2}}\left(\mathbb{R}^{n-k} \times \mathbb{R}^{k}\right)$, i.e.

$$
u(x)-\int e^{i x^{\prime} \xi^{\prime}} a\left(x^{\prime \prime}, \xi^{\prime}\right) d \xi^{\prime} \in G^{m_{2}-\rho_{2}} .
$$

Then we have for all $N \in \mathbb{N}_{0}$ an asymptotic symbol expansion

$$
\begin{array}{r}
b_{N}\left(x^{\prime \prime}, \xi^{\prime}\right)=\sum_{j<N}\left(\left\langle i D_{y^{\prime \prime}}, D_{\xi^{\prime \prime}}\right\rangle-\left\langle i D_{x^{\prime}}, D_{\xi^{\prime}}\right\rangle\right)^{j} p(x, \xi) a\left(y^{\prime \prime}, \xi^{\prime}\right) / j !_{y^{\prime \prime}=x^{\prime \prime}, x^{\prime}=\xi^{\prime \prime}=0} \\
\in S_{1,1}^{m_{1}+m_{2}, \min \left(\rho_{1}, \rho_{2}\right)}\left(\mathbb{R}^{n-k} \times \mathbb{R}^{k}\right)
\end{array}
$$

such that

$$
p(x, D) u(x)-\int e^{i x^{\prime}, \xi^{\prime}} b_{N}\left(x^{\prime \prime}, \xi^{\prime}\right) d \xi^{\prime} \in I_{1,1}^{m_{1}+m_{2}-N, \min \left(\rho_{1}, \rho_{2}\right)-N}\left(\mathbb{R}^{n}, \mathbb{R}^{n-k}\right) .
$$

Proof. We can cut out a remainder part $\hat{p}_{1-\chi}(\xi, \eta)=(1-\chi(\xi, \eta)) \hat{p}(\xi, \eta)$, with $p_{1-\chi} \in S_{1,1}^{m_{1}-\rho_{1}}$, whose contribution can be ignored due to Lemma 2.13, thus without loss of generality supp $\hat{p} \subseteq\{|\xi| \leq|\eta| / B \vee|\eta| \leq 1 / 2\}$. Analogously, we can cut out $\left.\hat{a}_{1-\chi}\left(\xi^{\prime \prime}, \xi^{\prime}\right)=\chi\left(\xi^{\prime \prime}, \xi^{\prime}\right) \hat{a}\left(\xi^{\prime \prime}, \xi^{\prime}\right)\right)$, with $a_{1-\chi} \in S_{1,1}^{m_{2}-\rho_{2}}$. Thus without loss of generality supp $\hat{a} \subseteq\left\{\left|\xi^{\prime \prime}\right| \leq\left|\xi^{\prime}\right| / B \vee\left|\xi^{\prime}\right| \leq 1 / 2\right\}$ for some $B>0$ chosen later. Furthermore we can without loss of generality assume that $N>\min \left(\rho_{1}, \rho_{2}\right)$.

Now we can split the equation via

$$
\begin{aligned}
p_{\nu}(x, \eta) & =p(x, \eta)\left(\psi_{\nu-1}+\psi_{\nu}+\psi_{\nu+1}+\psi_{\nu+2}\right)(\eta) \\
a_{\nu}\left(x^{\prime \prime}, \xi^{\prime}\right) & =a\left(x^{\prime \prime}, \xi^{\prime}\right) \psi_{\nu}\left(\xi^{\prime}\right) \\
(p \# a)\left(x^{\prime \prime}, \xi^{\prime}\right) & =\sum_{\nu}\left(p \# a_{\nu}\right)\left(x^{\prime \prime}, \xi^{\prime}\right)=\sum_{\nu}\left(p_{\nu} \# a_{\nu}\right)\left(x^{\prime \prime}, \xi^{\prime}\right) .
\end{aligned}
$$

Now with a rescaling scheme, we obtain

$$
P_{\nu}(x, \eta)=p_{\nu}\left(2^{-\nu} x, 2^{\nu} \eta\right) \quad A_{\nu}\left(x^{\prime \prime}, \xi^{\prime}\right)=a_{\nu}\left(2^{-\nu} x^{\prime \prime}, 2^{\nu} \xi^{\prime}\right) .
$$

With estimates

$$
\begin{aligned}
\left|\partial_{x}^{\alpha} \partial_{\eta}^{\beta} P_{\nu}(x, \eta)\right| & \lesssim 2^{\nu(|\beta|-|\alpha|)}\left\langle 2^{\nu} \eta\right\rangle^{m_{1}+\left(|\alpha|-\rho_{1}\right)^{+}-|\beta|} \\
& \lesssim 2^{\nu\left(m_{1}-\max \left(|\alpha|, \rho_{1}\right)\right)}\langle\eta\rangle^{-M} \\
\left|\partial_{x^{\prime \prime}}^{\alpha} \partial_{\xi^{\prime}}^{\beta} A_{\nu}\left(x^{\prime \prime}, \xi^{\prime}\right)\right| & \lesssim 2^{\nu(|\beta|-|\alpha|)}\left\langle 2^{\nu} \xi^{\prime}\right\rangle^{m_{2}+\left(|\alpha|-\rho_{2}\right)^{+}-|\beta|} \\
& \lesssim 2^{\nu\left(m_{2}-\max \left(|\alpha|, \rho_{2}\right)\right)}\left\langle\xi^{\prime}\right\rangle^{-M}
\end{aligned}
$$


Now applying standard theory of operators of type $(1,0)$, we obtain an asymptotic

$$
B_{\nu, N}\left(x^{\prime \prime}, \xi^{\prime}\right)=\sum_{j<N}\left(\left\langle i D_{y^{\prime \prime}}, D_{\xi^{\prime \prime}}\right\rangle-\left\langle i D_{x^{\prime}}, D_{\xi^{\prime}}\right\rangle\right)^{j} P_{\nu}(x, \xi) A_{\nu}\left(y^{\prime \prime}, \xi^{\prime}\right) / j !_{\left.\right|_{y^{\prime \prime}=x^{\prime \prime}, x^{\prime}=\xi^{\prime \prime}=0}}
$$

with

$$
R_{\nu, N}\left(x^{\prime \prime}, \xi^{\prime}\right)=\left(P_{\nu} \# A_{\nu}\right)\left(x^{\prime \prime}, \xi^{\prime}\right)-B_{\nu, N}\left(x^{\prime \prime}, \xi^{\prime}\right) \in S_{1,0}^{-2 M-N}
$$

with seminorms of $R_{\nu, N}$ bounded by seminorms of

$$
\left(\left\langle i D_{y^{\prime \prime}}, D_{\xi^{\prime \prime}}\right\rangle-\left\langle i D_{x^{\prime}}, D_{\xi^{\prime}}\right\rangle\right)^{N} P_{\nu}(x, \xi) A_{\nu}\left(y^{\prime \prime}, \xi^{\prime}\right) .
$$

Now with $N>\min \left(\rho_{1}, \rho_{2}\right)$, we will obtain

$$
\left|\partial_{x^{\prime \prime}}^{\alpha} \partial_{\xi^{\prime}}^{\beta} R_{\nu, N}\left(x^{\prime \prime}, \xi^{\prime}\right)\right| \lesssim 2^{\nu\left(m_{2}+m_{1}-\min \left(\rho_{1}, \rho_{2}\right)\right)}\left\langle\xi^{\prime}\right\rangle^{-2 M-N-|\beta|} .
$$

Considering the support properties, we obtain assuming $\nu \geq 0$ for $\xi^{\prime} \in \operatorname{supp} P_{\nu} \# A_{\nu}$

$$
\begin{aligned}
& \exists \xi^{\prime \prime} ; \xi \in \operatorname{supp} \widehat{P_{\nu} \# A_{\nu}} \Rightarrow \exists(\vartheta, \lambda) \in \operatorname{supp} \hat{P}_{\nu}(\lambda, \vartheta) \hat{A}_{\nu}(\vartheta) ; \vartheta+\lambda=\xi \\
& \Rightarrow 2 \geq|\vartheta| \geq B|\lambda| \wedge\left|\vartheta^{\prime}\right| \geq \frac{1}{2(1+1 / B)} \\
& \Rightarrow 2(1+1 / B) \geq\left|\xi^{\prime}\right| \geq \frac{1}{2(1+1 / B)}-2 / B .
\end{aligned}
$$

With $B \gg 0$ this is a compact interval away from 0 . Likewise we can assume this interval to be the $\xi^{\prime}$ support of $B_{\nu, N}$ and thus of $R_{\nu, N}$. Yielding for the back-scaled symbol

$$
r_{N}\left(x^{\prime \prime}, \xi^{\prime}\right)=\sum_{\nu} R_{\nu, N}\left(2^{\nu} x^{\prime \prime}, 2^{-\nu} \xi^{\prime}\right) \in S_{1,1}^{m_{1}+m_{2}-\min \left(\rho_{1}, \rho_{2}\right)} .
$$

Now observing that

$$
b_{N}\left(x^{\prime \prime}, \xi^{\prime}\right)=\sum_{\nu} B_{\nu, N}\left(2^{\nu} x^{\prime \prime}, 2^{-\nu} \xi^{\prime}\right)
$$

finishes the argument.

\section{Products, Paraproducts and the Taylor formula}

In this section we will understand how one can give an asymptotic expansion for products of conormal distributions. Note at first that the usual ansatz of a paramultiplication with the a priori Hölder-Zygmund estimates is insufficient, since even for type $(1,0)$ symbols obeying infinite improved regularity the corresponding paradifferential operator yields a symbol of finite improved regularity.

This is due to the anisotropic regularity of our distributions. The regularity in the $x^{\prime \prime}$-directions and in the $x^{\prime}$-direction are to be separated, which implies a corresponding adaptation to paraproducts.

Furthermore we will introduce a Taylor expansion in the case of codimension 1. There are several technical issues if one would want to extend this result to higher codimensions. Later on the Taylor expansion with remainder term will enable us to give an iterating scheme for deriving a symbolic solution to linearized problems. 
3.1. Taylor formula. We start the Taylor expansion with a simple continuation result.

Proposition 2.15. Let $v \in C_{*}^{\rho}\left(\mathbb{R}^{n-k}\right)$ with $\rho>C^{1}$ and let $\phi \in C_{c}^{\infty}\left(\mathbb{R}^{k}\right)$, with $\int \phi\left(\eta^{\prime}\right) d \eta^{\prime}=1$, be supported in an annulus, then

$$
[\mathfrak{e}(v)]\left(x^{\prime \prime}, \eta^{\prime}\right)=\sum_{\nu} \psi_{\nu}\left(D_{x^{\prime \prime}}\right) v\left(x^{\prime \prime}\right) 2^{-\nu k} \phi\left(2^{-\nu} \eta^{\prime}\right) \in S_{1,1}^{-\rho-k}\left(\mathbb{R}^{n-k} \times \mathbb{R}^{k}\right)
$$

is a symbol yielding an extension $\mathfrak{E}(v) \in I_{1,1}^{-\rho-k}\left(\mathbb{R}^{n}, \mathbb{R}^{n-k}\right)$ of $v$ via

$$
[\mathfrak{E}(v)](x)=\int e^{i x^{\prime} \eta^{\prime}}[\mathfrak{e}(v)]\left(x^{\prime \prime}, \eta^{\prime}\right) d \eta^{\prime} .
$$

Having further restrictions on $\phi$ we can deduce for $\alpha \in \mathbb{N}^{k} \rho>|\alpha|$

$$
\int\left(i \eta^{\prime}\right)^{\alpha} \phi\left(\eta^{\prime}\right) d \eta^{\prime}=0 \quad \Rightarrow \quad \partial_{x^{\prime}}^{\alpha}[\mathfrak{E}(v)]\left(x^{\prime \prime}, 0\right)=0 .
$$

Proof. Let $k_{-}, k_{+} \in \mathbb{Z}$ such that $\operatorname{supp} \phi \subseteq B_{2^{k_{+}}} \backslash B_{2^{k_{-}}}$. Let $\eta^{\prime}$ be given with $\left|\eta^{\prime}\right| \geq 2^{k_{-}-1}$, then we can restrict the sum to

$$
[\mathfrak{e}(v)]\left(x^{\prime \prime}, \eta^{\prime}\right)=\sum_{\nu=\left\lfloor\log _{2}\left(\eta^{\prime}\right)\right\rfloor+k_{-}-1}^{\left\lceil\log _{2}\left(\eta^{\prime}\right)\right\rceil+k_{+}+1} \psi_{\nu}\left(D_{x^{\prime \prime}}\right) v\left(x^{\prime \prime}\right) 2^{-\nu k} \phi\left(2^{-\nu} \eta^{\prime}\right)
$$

and if $\left|\eta^{\prime}\right|<2^{k_{-}-1}$, then $[\mathfrak{e}(v)]\left(x^{\prime \prime}, \eta^{\prime}\right)=0$. Now taking partial derivatives, we obtain

$$
\left|\partial_{x^{\prime \prime}}^{\alpha} \partial_{\eta^{\prime}}^{\beta}[\mathfrak{e}(v)]\left(x^{\prime \prime}, \eta^{\prime}\right)\right| \lesssim \sum_{\nu=\left\lfloor\log _{2}\left(\eta^{\prime}\right)\right\rfloor+k_{-}-1}^{\left\lceil\log _{2}\left(\eta^{\prime}\right)\right\rceil+k_{+}+1} 2^{\nu(|\alpha|-\rho-|\beta|-k)} \lesssim\left\langle\eta^{\prime}\right\rangle^{-\rho-k+|\alpha|-|\beta|}
$$

Thus $[\mathfrak{e}(v)]\left(x^{\prime \prime}, \eta^{\prime}\right) \in S_{1,1}^{-\rho-k}$. The property for the restriction follows immediately from $\int \phi\left(\eta^{\prime}\right) d \eta^{\prime}=1$ and $v\left(x^{\prime \prime}\right)=\sum_{\nu} \psi_{\nu}\left(D_{x^{\prime \prime}}\right) v\left(x^{\prime \prime}\right)$. Likewise the conclusion for the derivative is immediate.

For the case $k=1$ we will use this Proposition now extensively as a way to get a Taylor-like approximation of functions in $I_{1,1}^{m, \rho}$ with (non-constant) Taylor coefficients having full symbols in $S_{1,1}^{m-\rho+j}$, to stay in $G^{m-\rho}$.

Further we will use this continuation to show, that we can put more restrains on the remainder part of a conormal distribution. Namely with the following definition.

Definition 2.16. We define the following subspace $G_{0}^{\mu}\left(\mathbb{R}^{n}, \mathbb{R}^{n-k}\right) \subseteq G^{\mu}\left(\mathbb{R}^{n}, \mathbb{R}^{n-k}\right)$, with

$$
u \in G_{0}^{\mu}\left(\mathbb{R}^{n}, \mathbb{R}^{n-k}\right) \Leftrightarrow u \in G^{\mu}\left(\mathbb{R}^{n}, \mathbb{R}^{n-k}\right) \wedge \partial_{x^{\prime}}^{\alpha} u\left(x^{\prime \prime}, 0\right)=0 \quad \forall|\alpha|<-\mu-k
$$

Theorem 2.17 (Taylor expansion). Let $u \in I_{1,1}^{m, \rho}\left(\mathbb{R}^{n}, \mathbb{R}^{n-1}\right)$ with $\ell<-m-1 \leq \ell+1$ for some $\ell \geq 0$. Then introducing

$$
\mathfrak{E}_{j}(u)=\mathfrak{E}\left(\partial_{x^{\prime}}^{j} u\left(x^{\prime \prime}, 0\right)\right) / j ! \in I_{1,1}^{m-\rho+j} \quad \mathfrak{e}_{j}(u)=\mathfrak{e}\left(\partial_{x^{\prime}}^{j} u\left(x^{\prime \prime}, 0\right)\right) / j ! \in S_{1,1}^{m-\rho+j}
$$

there is a symbol $a_{\ell}^{\mathrm{r}} \in S_{1,1}^{m+\ell, \rho}$ with associated function $u_{\ell}^{\mathrm{r}}$ obeying $u_{\ell}^{\mathrm{r}}\left(x^{\prime \prime}, 0\right)=0$ and a remainder function $u_{G} \in G_{0}^{m-\rho}$ such that

$$
u(x)=\sum_{j \leq \ell}\left[\mathfrak{E}_{j}(u)\right](x)\left(x^{\prime}\right)^{j}+u_{\ell}^{\mathrm{r}}(x)\left(x^{\prime}\right)^{\ell}+u_{G}(x) .
$$

\footnotetext{
${ }_{1}$ Indeed this can be dropped if instead of $[\mathfrak{e}(v)]\left(x^{\prime \prime}, 0\right)=v\left(x^{\prime \prime}\right)$ we require $\lim _{\epsilon \rightarrow 0}[\mathfrak{e}(v)]\left(x^{\prime \prime}, \epsilon\right)=v\left(x^{\prime \prime}\right)$ as for $\rho \leq 0$ the trace is not well defined.
} 
Proof. First assume some decomposition of the form $u=u_{C}+\tilde{u}_{G}$, with $b\left(x^{\prime \prime}, \eta^{\prime}\right)$ as the full symbol for $u_{C}$. We can use Proposition 2.15 with a choice for $\phi$ such that (1) holds for all $|\alpha|<-m+\rho-1$, to extend

$$
\partial_{x^{\prime}}^{j} u_{C}\left(x^{\prime \prime}, 0\right) / j !=\int\left(i \eta^{\prime}\right)^{j} b\left(x^{\prime \prime}, \eta^{\prime}\right) \mathrm{d} \eta^{\prime} / j ! \in C_{*}^{-m+\rho-1-j}\left(\mathbb{R}^{n-1}\right)
$$

for all $j \leq \ell$. To understand the embedding observe

$$
\begin{aligned}
\left|\psi_{\nu}\left(D_{x^{\prime \prime}}\right) \partial_{x^{\prime}}^{j} u_{C}\left(x^{\prime \prime}, 0\right)\right| & \lesssim \int_{\left|\eta^{\prime}\right| \geq^{\nu}}\left|\eta^{\prime}\right|^{j} 2^{-\nu \rho}\left\langle\eta^{\prime}\right\rangle^{m} \mathrm{~d} \eta^{\prime}+\int_{\left|\eta^{\prime}\right|<2^{\nu}}\left|\eta^{\prime}\right|^{j} 2^{-\nu N}\left\langle\eta^{\prime}\right\rangle^{m+N-\rho} \mathrm{d} \eta^{\prime} \\
& \lesssim 2^{-\nu(-m+\rho-1-j)} .
\end{aligned}
$$

In parallel we can extend $\partial_{x^{\prime}}^{j} u_{G}\left(x^{\prime \prime}, 0\right) \in C_{*}^{-m+\rho-1-j}$ and obtain

$$
\mathfrak{e}_{j}(u)=\mathfrak{e}_{j}\left(u_{C}\right)+\mathfrak{e}_{j}\left(u_{G}\right) \in S_{1,1}^{m-\rho+j} .
$$

We can thus reduce the symbol $b$ via

$$
b^{r}\left(x^{\prime \prime}, \eta^{\prime}\right)=b\left(x^{\prime \prime}, \eta^{\prime}\right)-\sum_{j \leq \ell} D_{\eta^{\prime}}^{j}\left[\mathfrak{e}_{j}\left(u_{C}\right)\right]\left(x^{\prime \prime}, \eta^{\prime}\right) .
$$

With the property that for all $j \leq \ell$

$$
\int\left(i \eta^{\prime}\right)^{j} b^{r}\left(x^{\prime \prime}, \eta^{\prime}\right) \mathrm{d} \eta^{\prime}=0 .
$$

Now we define for all $0 \leq j \leq \ell-1$, taking $b_{0}^{r}=b^{r}$

$$
\begin{aligned}
b_{j+1}^{r}\left(x^{\prime \prime}, \eta^{\prime}\right) & =\int_{0}^{\infty}\left(i \zeta^{\prime}\right)^{j} / j ! b^{r}\left(x^{\prime \prime}, \eta^{\prime}-\zeta^{\prime}\right) \mathrm{d} \zeta^{\prime} \\
& =i \int_{0}^{\infty} \int_{0}^{\zeta^{\prime}}\left(i \xi^{\prime}\right)^{j-1} /(j-1) ! \mathrm{d} \xi^{\prime} b^{r}\left(x^{\prime \prime}, \eta^{\prime}-\zeta^{\prime}\right) \mathrm{d} \zeta^{\prime} \\
& =i \int_{0}^{\infty} \int_{0}^{\infty}\left(i \xi^{\prime}\right)^{j-1} /(j-1) ! b^{r}\left(x^{\prime \prime}, \eta^{\prime}-\lambda^{\prime}-\xi^{\prime}\right) \mathrm{d} \xi^{\prime} \mathrm{d} \lambda^{\prime} \\
& =i \int_{0}^{\infty} b_{j}^{r}\left(x^{\prime \prime}, \eta^{\prime}-\lambda^{\prime}\right) \mathrm{d} \lambda^{\prime} .
\end{aligned}
$$

And thus $\left(-i \partial_{\eta^{\prime}}\right) b_{j+1}^{r}\left(x^{\prime \prime}, \eta^{\prime}\right)=b_{j}^{r}\left(x^{\prime \prime}, \eta^{\prime}\right)$. Now we develop an alternative expression via

$$
\begin{aligned}
& \int\left(i \zeta^{\prime}\right)^{j} b^{r}\left(x^{\prime \prime}, \eta^{\prime}-\zeta^{\prime}\right) \mathrm{d} \zeta^{\prime}=\int\left(i\left(\eta^{\prime}-\xi^{\prime}\right)\right)^{j} b^{r}\left(x^{\prime \prime}, \xi^{\prime}\right) \mathrm{d} \xi^{\prime} \\
& =\sum_{k=0}^{j}\left(\begin{array}{l}
j \\
k
\end{array}\right)\left(i \eta^{\prime}\right)^{j-k} \int\left(i \xi^{\prime}\right)^{k} b^{r}\left(x^{\prime \prime}, \xi^{\prime}\right) \mathrm{d} \xi^{\prime}=0 .
\end{aligned}
$$

Thus

$$
b_{j+1}^{r}\left(x^{\prime \prime}, \eta^{\prime}\right)=-\int_{0}^{\infty}\left(i \zeta^{\prime}\right)^{j} / j ! b^{r}\left(x^{\prime \prime}, \eta^{\prime}+\zeta^{\prime}\right) \mathrm{d} \zeta^{\prime} .
$$

So to estimate symbol properties of $b_{\ell}^{r}\left(x^{\prime \prime}, \eta^{\prime}\right)$ it is sufficient to study $x^{\prime \prime}$-regularity behavior of $b_{j}^{r}$, since $\eta^{\prime}$ derivatives follow by induction. Due to the alternative expression we may assume in our estimates, that $\eta^{\prime} \leq 0$, else switch sign and the 
expression to still obtain the integral as a tail integral. Thus we obtain for all $m+s+j \leq 0$ if $1+\left|\eta^{\prime}\right|>2^{\nu}$

$$
\begin{aligned}
& \left|\psi_{\nu}\left(D_{x^{\prime \prime}}\right) b_{j}^{r}\left(x^{\prime \prime}, \eta^{\prime}\right)\right|=\left|\int_{0}^{\infty}\left(i \zeta^{\prime}\right)^{j-1} /(j-1) ! \psi_{\nu}\left(D_{x^{\prime \prime}}\right) b^{r}\left(x^{\prime \prime}, \eta^{\prime}-\zeta^{\prime}\right) \mathrm{d} \zeta^{\prime}\right| \\
& \lesssim \int_{0}^{1+\left|\eta^{\prime}\right|} 2^{-\nu(\rho+s)}\left|\zeta^{\prime}\right|^{j-1}\left(1+\left|\eta^{\prime}\right|\right)^{m+s} \mathrm{~d} \zeta^{\prime}+\int_{1+\left|\eta^{\prime}\right|}^{\infty} 2^{-\nu \rho}\left|\zeta^{\prime}\right|^{m+j-1} \mathrm{~d} \zeta^{\prime} \\
& \lesssim 2^{-\nu(\rho+s)}\left\langle\eta^{\prime}\right\rangle^{m+s+j}+2^{-\nu \rho}\left\langle\eta^{\prime}\right\rangle^{m+j} \lesssim 2^{-\nu(\rho+s)}\left\langle\eta^{\prime}\right\rangle^{m+s+j}
\end{aligned}
$$

and similarly for $1+\left|\eta^{\prime}\right| \leq 2^{\nu}$

$$
\begin{aligned}
& \left|\psi_{\nu}\left(D_{x^{\prime \prime}}\right) b_{j}^{r}\left(x^{\prime \prime}, \eta^{\prime}\right)\right|=\left|\int_{0}^{\infty}\left(i \zeta^{\prime}\right)^{j-1} /(j-1) ! \psi_{\nu}\left(D_{x^{\prime \prime}}\right) b^{r}\left(x^{\prime \prime}, \eta^{\prime}-\zeta^{\prime}\right) \mathrm{d} \zeta^{\prime}\right| \\
& \lesssim \int_{0}^{1+\left|\eta^{\prime}\right|} 2^{-\nu(\rho+s)}\left|\zeta^{\prime}\right|^{j-1}\left(1+\left|\eta^{\prime}\right|\right)^{m+s} \mathrm{~d} \zeta^{\prime}+\int_{1+\left|\eta^{\prime}\right|}^{2^{\nu}} 2^{-\nu(\rho+s+N)}\left|\zeta^{\prime}\right|^{m+j+s-1+N} \mathrm{~d} \zeta^{\prime} \\
& +\int_{2^{\nu}}^{\infty} 2^{-\nu \rho}\left|\zeta^{\prime}\right|^{m+j-1} \mathrm{~d} \zeta^{\prime} \\
& \lesssim 2^{-\nu(\rho+s)}\left\langle\eta^{\prime}\right\rangle^{m+s+j}+2^{-\nu(\rho-m-j)} \lesssim 2^{-\nu(\rho+s)}\left\langle\eta^{\prime}\right\rangle^{m+s+j} .
\end{aligned}
$$

Thus we obtain that $\partial_{\eta^{\prime}}^{k} b_{\ell}^{r}\left(x^{\prime \prime}, \eta^{\prime}\right) \in C_{*}^{-m+\rho-\ell+k} S_{1,1}^{m+\ell-k, \rho}\left(\mathbb{R}^{n}, \mathbb{R}^{n-1}\right)$ and by Proposition 2.7 the decomposition into the smooth summand $b_{\ell, 1}^{r} \in S_{1,1}^{m, \rho}\left(\mathbb{R}^{n}, \mathbb{R}^{n-1}\right)$ and the non smooth $b_{\ell, 2}^{r} \in C_{*}^{-m+\rho-\ell} S_{1,1}^{m+\ell-\rho}\left(\mathbb{R}^{n}, \mathbb{R}^{n-1}\right)$ is obtained with the improved symbol estimates

$$
\partial_{\eta^{\prime}}^{k} b_{\ell, 2}^{r} \in C_{*}^{-m+\rho-\ell+k} S_{1,1}^{m+\ell-k-\rho}\left(\mathbb{R}^{n}, \mathbb{R}^{n-1}\right) .
$$

Now we obtain

$$
u_{C}(x)=\sum_{j \leq \ell}\left(x^{\prime}\right)^{j} \mathfrak{E}_{j}\left(u_{C}\right)+\left(x^{\prime}\right)^{\ell} \int e^{i x^{\prime} \eta^{\prime}}\left(b_{\ell, 1}^{r}\left(x^{\prime \prime}, \eta^{\prime}\right)+b_{\ell, 2}^{r}\left(x^{\prime \prime}, \eta^{\prime}\right)\right) \mathrm{d} \eta^{\prime}
$$

with $b_{\ell, 1}^{r} \in S_{1,1}^{m+\ell, \rho}$. From Proposition 2.8 we obtain that

$$
u_{C, 2}^{r}(x)=\left(x^{\prime}\right)^{\ell} \int e^{i x^{\prime} \eta^{\prime}} b_{\ell, 2}^{r}\left(x^{\prime \prime}, \eta^{\prime}\right) \mathrm{d} \eta^{\prime} \in G^{m-\rho} .
$$

Thus $v_{G}=\tilde{u}_{G}-\sum_{j} \mathfrak{E}_{j}\left(\tilde{u}_{G}\right)\left(x^{\prime}\right)^{j}+u_{C, 2}^{r}(x) \in G^{m-\rho}$ with $\partial_{x^{\prime}}^{j} v_{G}\left(x^{\prime \prime}, 0\right)=0$ for all $j<\ell$, note that $u_{C, 2}^{r}$ a priori does not vanish of order $\ell$. Next, we can use Proposition 2.15 to obtain symbols $a_{j}^{G} \in S_{1,1}^{m-\rho+j}$ yielding an expansion of

$$
\partial_{x^{\prime}}^{j} v_{G}\left(x^{\prime \prime}, 0\right) \in C_{*}^{-m+\rho-1-j}\left(\mathbb{R}^{n-1}\right)
$$

for all $j<-m+\rho-1$. Then we can give

$$
\begin{aligned}
u_{G}(x) & =v_{G}(x)-\sum_{\ell \leq j<-m+\rho-1}\left[\mathfrak{E}_{j}\left(v_{G}\right)\right](x)\left(x^{\prime}\right)^{j} \\
a_{\ell}^{\mathrm{r}}\left(x^{\prime \prime}, \eta^{\prime}\right) & =b_{\ell, 1}^{r}\left(x^{\prime \prime}, \eta^{\prime}\right)+\sum_{\ell \leq j<-m+\rho-1} D_{\eta^{\prime}}^{j-\ell}\left[\mathfrak{e}_{j}\left(v_{G}\right)\right]\left(x^{\prime \prime}, \eta^{\prime}\right)
\end{aligned}
$$

which fulfill the requirements by construction. 
We also prove for codimension 1 that $G_{0}^{m, \ell}=\left(x^{\prime}\right) G_{0}^{m+1, \ell+1}$, which is handy in some computations.

Proposition 2.18. Let the codimension $k=1$, let $u \in G^{m, \ell}$ with $m<-2$, $u\left(x^{\prime \prime}, 0\right)=0$, then

$$
\left(x^{\prime}\right)^{-1} u(x) \in G^{m+1, \ell+1}
$$

Proof. As $\left(x^{\prime}\right)\left(x^{\prime}\right)^{-1} u(x)=u(x) \in G^{m, \ell}$, the we only thing we need to show is $\left(x^{\prime}\right)^{-1} u(x) \in G^{m+1}$. Which is equivalent to

$$
\left\|\psi_{\nu}(D)\left(x^{\prime}\right)^{-1} u(x)\right\|_{L^{p}} \lesssim 2^{\nu(m+2-1 / p)}
$$

for all $1<p \leq \infty$ with constants depending on $p$. As $u\left(x^{\prime \prime}, 0\right)=0$ we have

$$
\left(x^{\prime}\right)^{-1} u(x)=\left(x^{\prime}\right)^{-1}\left(u(x)-u\left(x^{\prime \prime}, 0\right)\right)=\int_{0}^{1} \partial_{x^{\prime}} u\left(x^{\prime \prime}, s x^{\prime}\right) \mathrm{d} s
$$

Now let $v(x)=\partial_{x^{\prime}} u(x)$, then

$$
\begin{aligned}
\psi_{\nu}(D) v\left(x^{\prime \prime}, s x^{\prime}\right) & =\left[\psi_{\nu}\left(D_{x^{\prime \prime}}, s D_{y^{\prime}}\right) v\left(x^{\prime \prime}, y^{\prime}\right)\right]_{y^{\prime}=s x^{\prime}} \\
& =\left[\psi_{\nu}\left(D_{x^{\prime \prime}}, s D_{y^{\prime}}\right) \sum_{\mu \geq \nu-2} \psi_{\mu}\left(D_{x^{\prime \prime}}, D_{y^{\prime}}\right) v\left(x^{\prime \prime}, y^{\prime}\right)\right]_{y^{\prime}=s x^{\prime}}
\end{aligned}
$$

Now we have from $u \in G^{m}$, that

$$
\left\|\psi_{\mu}(D) v\right\|_{L^{p}} \lesssim 2^{\mu(m+2-1 / p)} \Rightarrow \sum_{\mu \geq \nu-2}\left\|\psi_{\mu}(D) v\right\|_{L^{p}} \lesssim 2^{\nu(m+2-1 / p)}
$$

Now as $\psi_{\nu}\left(D_{x^{\prime \prime}}, s D_{y^{\prime}}\right)$ is uniformly $L^{p}$ continuous for $0 \leq s \leq 1$, we obtain

$$
\left\|\psi_{\nu}\left(D_{x^{\prime \prime}}, s D_{y^{\prime}}\right) v\left(x^{\prime \prime}, y^{\prime}\right)\right\|_{L^{p}} \lesssim 2^{\nu(m+2-1 / p)}
$$

And thus by scaling, we obtain

$$
\left\|\psi_{\nu}(D) v\left(x^{\prime \prime}, s x^{\prime}\right)\right\|_{L^{p}} \lesssim s^{-1 / p} 2^{\nu(m+2-1 / p)}
$$

And we obtain

$$
\left\|\psi_{\nu}(D)\left(x^{\prime}\right)^{-1} u(x)\right\|_{L^{p}} \lesssim \int_{0}^{1} s^{-1 / p} 2^{\nu(m+2-1 / p)} \mathrm{d} s \lesssim \frac{1}{1-1 / p} 2^{\nu(m+2-1 / p)}
$$

Which provides the claim.

3.2. Products and Paraproducts. To approach the main theorem of this section introduce the cutoff function $\chi(\zeta, \eta) \in S^{0}$ with the following properties.

$$
\begin{aligned}
\operatorname{supp} \chi(\zeta, \eta) & \subseteq\{|\zeta| \leq B(|\eta|+1),|\eta| \leq B(|\zeta+\eta|+1)\} \\
\chi(\zeta, \eta) & =1 \quad \forall|\eta| \geq B(|\zeta|+1)
\end{aligned}
$$

for some $B>2$. Then we can introduce

$$
\Phi(\zeta, \eta)=1-\chi(\zeta, \eta)-\chi(\eta, \zeta)
$$

with the properties

$$
\begin{aligned}
& \operatorname{supp} \Phi \subseteq\{|\zeta| \geq B(|\eta|+1),|\eta| \geq B(|\eta|+1)\} \\
& \Phi(\zeta, \eta)=1 \quad \forall B(|\eta+\zeta|+1) \leq \min (|\zeta|,|\eta|)
\end{aligned}
$$


An example of such cutoff functions are for some $d \geq 3$

$$
\begin{aligned}
\chi(\zeta, \eta) & =\sum_{i+d<j} \psi_{i}(\zeta) \psi_{j}(\eta)=\sum_{i \geq 0} \varphi_{i}(\zeta) \psi_{i+d}(\eta) \\
\Phi(\zeta, \eta) & =\sum_{|i-j| \leq d} \psi_{i}(\zeta) \psi_{j}(\eta)
\end{aligned}
$$

These cutoff functions are used to obtain a paraproduct. Observing that the Fourier transform can be split into these cutoff regions

$$
\widehat{u v}(\xi)=\int \hat{u}(\xi-\eta)(\chi(\xi-\eta, \eta)+\chi(\eta, \xi-\eta)+\Phi(\xi-\eta, \eta)) \hat{v}(\eta) d \eta,
$$

we can extract the formulas for the paradifferential operators given by the symbols

$$
\begin{gathered}
u_{\chi}(x, \eta)=\chi(D, \eta) u(x)=\int e^{i x \xi} \chi(\xi, \eta) \hat{u}(\xi) \mathrm{d} \xi \\
u_{\Phi}(x, \eta)=\Phi(D, \eta) u(x)=\int e^{i x \xi} \Phi(\xi, \eta) \hat{u}(\xi) \mathrm{d} \xi .
\end{gathered}
$$

And thus we obtain the decomposition

$$
\begin{aligned}
u(x) v(x) & =u_{\chi}(x, D) v(x)+v_{\chi}(x, D) u(x)+u_{\Phi}(x, D) v(x) \\
& =u_{\chi}(x, D) v(x)+v_{\chi}(x, D) u(x)+v_{\Phi}(x, D) u(x) .
\end{aligned}
$$

In an abuse of notation, we also introduce for symbols

$$
\begin{aligned}
a_{\chi}\left(x, \xi^{\prime}\right) & =\int e^{i x^{\prime} \zeta^{\prime}} a\left(x^{\prime \prime}, \zeta^{\prime}\right) \chi\left(\zeta^{\prime}, \xi^{\prime}\right) \mathrm{d} \zeta^{\prime} \\
a_{\Phi}\left(x, \xi^{\prime}\right) & =\int e^{i x^{\prime} \zeta^{\prime}} a\left(x^{\prime \prime}, \zeta^{\prime}\right) \Phi\left(\zeta^{\prime}, \xi^{\prime}\right) \mathrm{d} \zeta^{\prime} .
\end{aligned}
$$

The rest of the section is devoted to provide subresults yielding the following main theorem.

THeOREM 2.19. Let $u \in I_{1,1}^{m_{1}, \rho_{1}}$ and $v \in I_{1,1}^{m_{2}, \rho_{2}}$ with $m_{i}+1<0, m_{i} \notin \mathbb{Z}$ and $m_{1} \geq m_{2}$, where $u$ has full symbol $a_{1}$ and $b$ has full symbol $a_{2}$. Let $\ell_{i} \in \mathbb{N}_{0}$ be maximal with the property $m_{i}+1+\ell_{i}<0$. We can give the following approximation for a product

$$
\begin{aligned}
u(x) v(x) & =\sum_{i=0}^{\ell_{1}} \sum_{j=0}^{\ell_{1}-i} \frac{\left(x^{\prime}\right)^{i+j}}{i ! j !} \mathfrak{E}\left(\left(\partial_{x^{\prime}}^{i} u\left(x^{\prime \prime}, 0\right)\right) \partial_{x^{\prime}}^{j} v\left(x^{\prime \prime}, 0\right)\right) \\
& +\int e^{i x^{\prime} \xi^{\prime}} \sum_{i=0}^{\ell_{1}} D_{\xi^{\prime}}^{i}\left(\left[\mathfrak{r}_{i}(u)\right]_{\chi}\left(x^{\prime \prime}, \xi^{\prime}\right) D_{\xi^{\prime}}^{\ell_{2}} a_{2, \ell_{2}}^{\mathrm{r}}\left(x^{\prime \prime}, \xi^{\prime}\right)\right) d \xi^{\prime} \\
& +\int e^{i x^{\prime}, \xi^{\prime}} \sum_{j=0}^{\ell_{2}} D_{\xi^{\prime}}^{j}\left(\left[\mathfrak{e}_{i}(v)\right]_{\chi}\left(x^{\prime \prime}, \xi^{\prime}\right) D_{\xi^{\prime}}^{\ell_{1}} \mathrm{a}_{1, \ell_{1}}^{\mathrm{r}}\left(x^{\prime \prime}, \xi^{\prime}\right)\right) d \xi^{\prime}+r(x)
\end{aligned}
$$

with $r(x) \in I_{1,1}^{m_{1}+m_{2}+k, \min \left(\rho_{i}\right)}$ sufficing $\partial_{x^{\prime}}^{j} r\left(x^{\prime \prime}, 0\right)=0, \forall j \leq \ell_{1}$.

In some of our following statements we are going to be a bit wasteful on the precise improved regularity of the component. This is due to the fact that these terms reach below the threshold of $\max \left(m_{i}-\rho_{i}\right)$ in their remainder terms and those are neglectable in combination with other terms. 
Firstly we are going to apply the standard paramultiplication following the description in $\left[\mathbf{H o ̈ 0 3 b}\right.$, Section 10.2.]. We will establish, that any term in $G_{0}^{m-\rho}$ can be neglected within a multiplication regarding the product up to $G_{0}^{m-\rho}$.

In the general paramultiplication scheme as described above, we obtain $u_{\chi}(x, \eta) \in$ $S_{1,1}^{0, \rho}$ and $u_{\Phi}(x, \eta) \in S_{1,1}^{-\rho}$ if $u \in C_{*}^{\rho}$, and as an aside moreover we will obtain that for $b(x, D)=u_{\chi}(x, D)^{*}$ we have $b(x, \eta) \in S_{1,1}^{m, \rho}$, which we will not correspondingly get for $u_{\Phi}(x, D)^{*}$. Again we refer to $[\mathbf{H o ̈ 0 3 b}$, Section 10.2.] for more details. Using this we prove the following Proposition.

Proposition 2.20. Let $u \in I_{1,1}^{m, \rho}\left(\mathbb{R}^{n}, \mathbb{R}^{n-k}\right)$ with $m<-k$ and $v \in G_{0}^{m-\rho}$, then

$$
u(x) v(x) \in G_{0}^{m-\rho}
$$

Proof. Using paramultiplication, we obtain immediately with $u \in C_{*}^{-m-k}$

$$
\left(u_{\chi}(x, D)+u_{\Phi}(x, D)\right) v \in G^{m-\rho}
$$

Thus we need to investigate $v_{\chi}(x, \eta)$ and as $\partial_{x}^{\alpha} v\left(x^{\prime \prime}, 0\right)=0$ for all $|\alpha| \leq N$ with $N \in \mathbb{N}$ such that $\rho<N<-m+\rho-k$, we can compute

$$
\begin{aligned}
\left|\partial_{x}^{\alpha} \partial_{\eta}^{\beta} v_{\chi}\left(x^{\prime \prime}, 0, \eta\right)\right| & =\left|\partial_{\eta}^{\beta} \chi(D, \eta) \partial_{x}^{\alpha} v\left(x^{\prime \prime}, 0\right)\right|=\left|-\partial_{\eta}^{\beta}(1-\chi(D, \eta)) \partial_{x}^{\alpha} v\left(x^{\prime \prime}, 0\right)\right| \\
& \lesssim\langle\eta\rangle^{-|\beta|-N+|\alpha|}
\end{aligned}
$$

Note that as we a priori have $u_{\chi} \in S_{1,1}^{0,-m+\rho-k}$ and thus for $|\alpha|>N$ we obtain

$$
\left|\partial_{x}^{\alpha} \partial_{\eta}^{\beta} u_{\chi}(x, \eta)\right| \lesssim\langle\eta\rangle^{-|\beta|+|\alpha|-N}
$$

We conclude that $\partial_{x^{\prime}}^{\alpha} u_{\chi}\left(x^{\prime \prime}, 0, \eta\right) \in S_{1,1}^{-N+|\alpha|}\left(\mathbb{R}^{n-k} \times \mathbb{R}^{k}\right)$. And thus by Theorem 2.14 we obtain symbol terms in $S_{1,1}^{-N+m}$ within the expansion of

$$
\begin{aligned}
v_{\chi}(x, \eta) \# a\left(x^{\prime \prime}, \eta^{\prime}\right) & =\sum_{j<N}\left(\left\langle i D_{y^{\prime \prime}} D_{\xi^{\prime \prime}}\right\rangle-\left\langle i D_{x^{\prime}} D_{\xi^{\prime}}\right\rangle\right)^{j} v_{\chi}(x, \xi) a\left(y^{\prime \prime}, \xi^{\prime}\right) / j !_{\substack{y^{\prime \prime}=x^{\prime \prime} \\
x^{\prime}=\xi^{\prime}=0}} \\
& +r_{N}\left(x^{\prime \prime}, \xi^{\prime}\right) \\
\int e^{i x^{\prime} \xi^{\prime}} r_{N}\left(x^{\prime \prime}, \xi^{\prime}\right) \mathrm{d} \xi^{\prime} & \in I_{1,1}^{m-N, \rho-N}=I_{1,1}^{m-\rho} \\
\Rightarrow \quad v_{\chi}(x, D) u & \in G^{m-\rho}
\end{aligned}
$$

Thus it remains to argue that $u(x) v(x)$ vanishes at $x^{\prime}=0$ of order $M$ with $M<$ $-m-k+\rho$ maximal. But as $u \in C_{*}^{-m-k} \subseteq C^{0}$ this follows immediately from $v$ vanishing of oder $M$ there.

Thus all the relevant parts of multiplication takes place for symbols only. We are therefore able to alter the scheme of paramultiplication to take place only in $\eta^{\prime}$ in order to sidestep some anisotropic improved regularity issues. Then we can analogously split

$$
\begin{aligned}
& \int a\left(x^{\prime \prime}, \zeta^{\prime}\right) b\left(x^{\prime \prime}, \xi^{\prime}-\zeta^{\prime}\right) \mathrm{d} \zeta^{\prime} \\
= & \int a\left(x^{\prime \prime}, \zeta^{\prime}\right)\left(\chi\left(\zeta^{\prime}, \xi^{\prime}-\zeta^{\prime}\right)+\chi\left(\xi^{\prime}-\zeta^{\prime}, \zeta^{\prime}\right)+\Phi\left(\zeta^{\prime}, \xi^{\prime}-\zeta^{\prime}\right)\right) b\left(x^{\prime \prime}, \xi^{\prime}-\zeta^{\prime}\right) \mathrm{d} \zeta^{\prime}
\end{aligned}
$$

We first restate the symbol expansion formula for paradifferential operators acting on conormal distributions for this kind of operators. They evidently have anisotropic improved regularity but to have a $x^{\prime}$-dependent symbol is mainly for consistency of notation, in most computations we will stick with the symbols. 
Note that we can capture the behavior of $a_{\chi}$, which is the standard symbol behavior away from $m+k=0$ with a $\log \left(\left\langle\eta^{\prime}\right\rangle\right)$ term at $m+k=0$ distorting the estimates in a neighborhood of $m+k=0$, with the following remark.

REMARK 2.21. Let $a\left(x^{\prime \prime}, \eta^{\prime}\right) \in S_{1,1}^{m, \rho}$, then $a_{\chi}\left(x, \eta^{\prime}\right)_{\left.\right|_{x^{\prime}=0}}$ satisfies

$$
\left|\psi_{\nu}\left(D_{x^{\prime \prime}}\right) \partial_{\eta^{\prime}}^{\beta} a_{\chi}\left(x, \eta^{\prime}\right)_{\left.\right|_{x^{\prime}=0}}\right| \lesssim 2^{-\nu \rho} \begin{cases}2^{-\nu M}\left\langle\eta^{\prime}\right\rangle^{m+M+k} & \left\langle\eta^{\prime}\right\rangle \leq 2^{\nu} \\ 2^{\nu(m+k)}\left(1+\frac{\left(2^{-\nu}\left\langle\eta^{\prime}\right\rangle\right)^{m+k}-1}{m+k}\right) & \left\langle\eta^{\prime}\right\rangle \geq 2^{\nu}, m \neq-k \\ 1+\log \left(2^{-\nu}\left\langle\eta^{\prime}\right\rangle\right) & \left\langle\eta^{\prime}\right\rangle \geq 2^{\nu}, m=-k\end{cases}
$$

But as we are not going to thoroughly study the neighborhood of $m+k=0$, we do not give a proof of this assertion. First we give the case $m_{1}+k>0$, which yields only a qualitative statement and does not allow a symbol approximation.

Proposition 2.22. Let $a\left(x^{\prime \prime}, \eta^{\prime}\right) \in S_{1,1}^{m_{1}, \rho_{1}}$ and $b\left(x^{\prime \prime}, \eta^{\prime}\right) \in S_{1,1}^{m_{2}, \rho_{2}}$ with $m_{1}+k>0$, then

$$
c\left(x^{\prime \prime}, \xi^{\prime}\right)=\int a\left(x^{\prime \prime}, \zeta^{\prime}\right) \chi\left(\zeta^{\prime}, \xi^{\prime}-\zeta^{\prime}\right) b\left(x^{\prime \prime}, \xi^{\prime}-\zeta^{\prime}\right) d \zeta^{\prime} \in S_{1,1}^{m_{1}+m_{2}+k, \min \left(\rho_{i}\right)}
$$

Proof. Due to the support properties of $\chi$, we can see that on the support of the integrand we have $\left\langle\xi^{\prime}-\zeta^{\prime}\right\rangle \sim\left\langle\xi^{\prime}\right\rangle$, since we directly have $\left|\xi^{\prime}-\zeta\right| \leq B\left(\left|\xi^{\prime}\right|+1\right)$ and the converse is obtained if $\left|\zeta^{\prime}\right| \leq\left|\xi^{\prime}\right| / 2$ by $\left|\xi^{\prime}-\zeta^{\prime}\right| \geq\left|\xi^{\prime}\right|-\left|\zeta^{\prime}\right|$ and if $\left|\zeta^{\prime}\right| \geq\left|\xi^{\prime}\right| / 2$ by $B\left(\left|\xi^{\prime}-\zeta^{\prime}\right|+1\right) \geq\left|\zeta^{\prime}\right|$. Thus we also have $\left|\zeta^{\prime}\right| \lesssim\left\langle\xi^{\prime}\right\rangle$ on the support of the integrand, making it compact. We can then estimate

$$
\begin{aligned}
\left|\psi_{\nu}\left(D_{x^{\prime \prime}}\right) \partial_{\xi^{\prime}}^{\beta} c\left(x^{\prime \prime}, \xi^{\prime}\right)\right| & \lesssim \int_{\left|\zeta^{\prime}\right| \lesssim\left\langle\xi^{\prime}\right\rangle} 2^{-\nu \rho_{1}}\left\langle\zeta^{\prime}\right\rangle^{m_{1}}\left\langle\xi^{\prime}\right\rangle^{m_{2}-|\beta|}+\left\langle\zeta^{\prime}\right\rangle^{m_{1}} 2^{-\nu \rho_{2}}\left\langle\xi^{\prime}\right\rangle^{m_{2}-|\beta|} \mathrm{d} \zeta^{\prime} \\
& \lesssim 2^{-\nu \min \left(\rho_{i}\right)}\left\langle\xi^{\prime}\right\rangle^{m_{1}+m_{2}+k}
\end{aligned}
$$

And analogously for spacial derivatives $\partial_{x^{\prime \prime}}^{\alpha}$ with $|\alpha|>\min \left(\rho_{i}\right)$, we obtain

$$
\begin{aligned}
\left|\partial_{x^{\prime \prime}}^{\alpha} \partial_{\xi^{\prime}}^{\beta} c\left(x^{\prime \prime}, \xi^{\prime}\right)\right| & \lesssim \int_{\left|\zeta^{\prime}\right| \lesssim\left\langle\xi^{\prime}\right\rangle}\left\langle\zeta^{\prime}\right\rangle^{m_{1}}\left\langle\xi^{\prime}\right\rangle^{m_{2}-|\beta|+|\alpha|-\min \left(\rho_{i}\right)} \mathrm{d} \zeta^{\prime} \\
& \lesssim\left\langle\xi^{\prime}\right\rangle^{m_{1}+m_{2}+k-|\beta|+|\alpha|-\min \left(\rho_{i}\right)}
\end{aligned}
$$

For the case $m_{1}+k<0$ we are able to give a symbol expansion.

Proposition 2.23. Let $a\left(x^{\prime \prime}, \eta^{\prime}\right) \in S_{1,1}^{m_{1}, \rho_{1}}$ and $b\left(x^{\prime \prime}, \eta^{\prime}\right) \in S_{1,1}^{m_{2}, \rho_{2}}$ with $m_{1}+k<0$ and $\epsilon>0$, then for $N \geq 0$ define the approximation

$$
b_{N}\left(x^{\prime \prime}, \xi^{\prime}\right)=\sum_{|\alpha|<N}\left(-\partial_{x^{\prime}}\right)^{\alpha} D_{\xi^{\prime}}^{\alpha} a_{\chi}\left(x, \xi^{\prime}\right) b\left(x^{\prime \prime}, \xi^{\prime}\right) / \alpha !_{x^{\prime}=0} \in S_{1,1}^{m_{2}, \rho}
$$

with. $^{2} \rho=\min \left(\rho_{2}, \rho_{1}-m_{1}-k-\epsilon\right)$ and we obtain the estimate

$$
r_{N}\left(x^{\prime \prime}, \xi^{\prime}\right)=\int a\left(x^{\prime \prime}, \zeta^{\prime}\right) \chi\left(\zeta^{\prime}, \xi^{\prime}-\zeta^{\prime}\right) b\left(x^{\prime \prime}, \xi^{\prime}-\zeta^{\prime}\right) d \zeta^{\prime}-b_{N}\left(x^{\prime \prime}, \xi^{\prime}\right) \in S_{1,1}^{m_{2}-s_{N}, \rho-s_{N}}
$$

with $s_{N}=\min \left(N,-m_{1}-k\right)-\epsilon_{N}$, where $\epsilon_{N}=\left(N+m_{1}+k\right)_{\epsilon}^{\Delta}$.

\footnotetext{
${ }^{2}$ In fact dropping $\epsilon$ in the equation for $\rho$, we would only encounter a $\log \left\langle\xi^{\prime}\right\rangle$ factor in the symbol
} estimates at the critical regularity level. Nevertheless this version is sufficient for the multiplication. 
ProOF. For computations one should first restate the approximation $b_{N}$ in terms of symbols, id est

$$
b_{N}\left(x^{\prime \prime}, \xi^{\prime}\right)=\sum_{|\alpha|<N} \int\left(-i \zeta^{\prime}\right)^{\alpha}\left(-i \partial_{\xi^{\prime}}\right)^{\alpha} a\left(x^{\prime \prime}, \zeta^{\prime}\right) \chi\left(\zeta^{\prime}, \xi^{\prime}\right) b\left(x^{\prime \prime}, \xi^{\prime}\right) / \alpha ! d \zeta^{\prime}
$$

As $\left\langle\zeta^{\prime}\right\rangle \lesssim\left\langle\xi^{\prime}\right\rangle$ on the support of $\chi$, we can - for the first symbol estimate - restrict the case to $|\alpha|=0$. Then we obtain

$$
\begin{aligned}
& 2^{\nu \mu}\left|\psi_{\nu}\left(D_{x^{\prime \prime}}\right) \partial_{\xi^{\prime}}^{\beta} b_{N}\left(x^{\prime \prime}, \xi^{\prime}\right)\right| \\
& \lesssim \int_{\left\langle\zeta^{\prime}\right\rangle \lesssim\left\langle\xi^{\prime}\right\rangle}\left\langle\zeta^{\prime}\right\rangle^{m_{1}+\left(\mu-\rho_{1}\right)^{+}}\left\langle\xi^{\prime}\right\rangle^{m_{2}-|\beta|}+\left\langle\zeta^{\prime}\right\rangle^{m_{1}}\left\langle\xi^{\prime}\right\rangle^{m_{2}+\left(\mu-\rho_{2}\right)^{+-}-|\beta|} \mathrm{d} \zeta^{\prime} \\
& \lesssim\left\langle\xi^{\prime}\right\rangle^{m_{2}+\left(\mu+m_{1}-\rho_{1}+k+\epsilon\right)^{+}-|\beta|}+\left\langle\xi^{\prime}\right\rangle^{m_{2}+\left(\mu-\rho_{2}\right)^{+-}|\beta|} \lesssim\left\langle\xi^{\prime}\right\rangle^{m_{2}+(\mu-\rho)^{+-}-|\beta|}
\end{aligned}
$$

For $r_{N}\left(x^{\prime \prime}, \xi^{\prime}\right)$ we can use the Taylor remainder formula with uniform bounds on the derivatives for $\left|\zeta^{\prime}\right|<\left|\eta^{\prime}\right| / 2$.

$$
\begin{aligned}
& 2^{\nu \mu}\left|\psi_{\nu}\left(D_{x^{\prime \prime}}\right) \partial_{\xi^{\prime}}^{\beta} r_{N}\left(x^{\prime \prime}, \xi^{\prime}\right)\right| \\
& \lesssim \int_{\left|\zeta^{\prime}\right| \lesssim\left|\xi^{\prime}\right|}\left\langle\zeta^{\prime}\right\rangle^{m_{1}+N+\left(\mu-\rho_{1}\right)^{+}}\left\langle\xi^{\prime}\right\rangle^{m_{2}-|\beta|-N}+\left\langle\zeta^{\prime}\right\rangle^{m_{1}+N}\left\langle\xi^{\prime}\right\rangle^{m_{2}-|\beta|-N+\left(\mu-\rho_{2}\right)^{+}} \mathrm{d} \zeta^{\prime} \\
& +\int_{\left|\zeta^{\prime}\right| \sim\left|\xi^{\prime}\right|} \sum_{|\alpha|<N}\left\langle\zeta^{\prime}\right\rangle^{m_{1}+|\alpha|+\left(\mu-\rho_{1}\right)^{+}}\left\langle\xi^{\prime}\right\rangle^{m_{2}-|\beta|-|\alpha|}+\left\langle\zeta^{\prime}\right\rangle^{m_{1}+|\alpha|}\left\langle\xi^{\prime}\right\rangle^{m_{2}-|\beta|-|\alpha|+\left(\mu-\rho_{2}\right)^{+}} \mathrm{d} \zeta^{\prime} \\
& +\int_{\left|\zeta^{\prime}\right| \sim\left|\xi^{\prime}-\zeta^{\prime}\right| \sim\left|\xi^{\prime}\right|}\left\langle\zeta^{\prime}\right\rangle^{m_{1}+\left(\mu-\rho_{1}\right)^{+}}\left\langle\xi^{\prime}-\zeta^{\prime}\right\rangle^{m_{2}-|\beta|}+\left\langle\zeta^{\prime}\right\rangle^{m_{1}}\left\langle\xi^{\prime}-\zeta^{\prime}\right\rangle^{m_{2}-|\beta|+\left(\mu-\rho_{2}\right)^{+}} \mathrm{d} \zeta^{\prime} \\
& \lesssim\left\langle\xi^{\prime}\right\rangle^{\left(m_{1}+k+N+\left(\mu+\epsilon-\rho_{1}\right)^{+}\right)^{+}+\epsilon_{N}+m_{2}-|\beta|-N}+\left\langle\xi^{\prime}\right\rangle^{\left(m_{1}+k+N\right)^{+}+\epsilon_{N}+m_{2}-|\beta|-N+\left(\mu-\rho_{2}\right)^{+}} \\
& +\left\langle\xi^{\prime}\right\rangle^{m_{1}+m_{2}+k-|\beta|+\left(\mu-\min \left(\rho_{1}, \rho_{2}\right)\right)^{+}} \\
& \lesssim\left\langle\xi^{\prime}\right\rangle^{m_{2}-s_{N}+\left(\mu-\rho+s_{N}\right)^{+}-|\beta|}
\end{aligned}
$$

So having $m \in \mathbb{Z}$ results in a loss of approximation quality at the point where $\partial_{x^{\prime}}^{\alpha} u\left(x^{\prime \prime}, x^{\prime}\right)$ is no longer continuous, e.g. the Heaviside function. This special case and some others might probably be overcome by other methods, which utilize their boundedness.

To give an expansion of the product with an error term in $S_{1,1}^{m_{1}+m_{2}+k, \min \left(\rho_{i}\right)}$ for the important case of $m_{i} \notin \mathbb{Z}$ and $m_{i}+k<0$ we are left with estimating the $\Phi$ term in the decomposition, which itself does not have a symbol expansion.

Proposition 2.24. Let $a\left(x^{\prime \prime}, \eta^{\prime}\right) \in S_{1,1}^{m_{1}, \rho_{1}}, b\left(x^{\prime \prime}, \eta^{\prime}\right) \in S_{1,1}^{m_{2}, \rho_{2}}$ with $m_{1}+m_{2}+k<0$ then we have

$$
a_{\Phi}\left(x, D_{x^{\prime}}\right) \int e^{i x^{\prime} \xi^{\prime}} b\left(x^{\prime \prime}, \xi^{\prime}\right) d \xi^{\prime} \in I_{1,1 ; \infty}^{m_{1}+m_{2}+k, \min \left(\rho_{i}\right)}
$$


Proof. First note, that on the support of $\Phi\left(\zeta^{\prime}, \xi^{\prime}-\zeta^{\prime}\right)$ we have $\left\langle\zeta^{\prime}\right\rangle \approx\left\langle\xi^{\prime}-\zeta^{\prime}\right\rangle$ and thus $\left\langle\zeta^{\prime}\right\rangle \gtrsim\left\langle\eta^{\prime}\right\rangle$, which yields the estimates for $\mu<-m_{1}-m_{2}-k+|\beta|+\min \left(\rho_{i}\right)$ :

$$
\begin{aligned}
& \left|\partial_{\xi^{\prime}}^{\beta} \psi_{\nu}\left(D_{x^{\prime \prime}}\right) \int a\left(x^{\prime \prime}, \zeta^{\prime}\right) \Phi\left(\zeta^{\prime}, \xi^{\prime}-\zeta^{\prime}\right) b\left(x^{\prime \prime}, \xi^{\prime}-\zeta^{\prime}\right) \mathrm{d} \zeta^{\prime}\right| \\
& \lesssim 2^{-\nu \mu} \int_{\left\langle\zeta^{\prime}\right\rangle \gtrsim\left\langle\eta^{\prime}\right\rangle}\left\langle\zeta^{\prime}\right\rangle^{m_{1}+m_{2}+\left(\mu-\rho_{2}\right)^{+}-|\beta|}+\left\langle\zeta^{\prime}\right\rangle^{m_{1}+\left(\mu-\rho_{1}\right)^{+}+m_{2}-|\beta|} \mathrm{d} \zeta^{\prime} \\
& \lesssim 2^{-\nu \mu} \frac{\left\langle\xi^{\prime}\right\rangle^{m_{1}+m_{2}+k+\left(\mu-\min \left(\rho_{i}\right)\right)^{+}-|\beta|}}{-m_{1}-m_{2}-k-\left(\mu-\min \left(\rho_{i}\right)\right)^{+}+|\beta|} .
\end{aligned}
$$

Combined with Proposition 2.7 and 2.8 this yields the claim.

We conclude the results so far in the following Corollary.

Corollary 2.25. Let $u \in I_{1,1}^{m_{1}, \rho_{1}}$ and $v \in I_{1,1}^{m_{2}, \rho_{2}}$ with $m_{1}+m_{2}+k<0$, and without loss of generality $m_{1} \leq m_{2}$, then

$$
u v(x) \in I_{1,1}^{m, \rho}
$$

with

$$
\begin{aligned}
m & = \begin{cases}m_{1}+m_{2}+k & m_{1}+k>0 \\
m_{2}+\epsilon & m_{1}+k=0 \\
m_{2} & m_{1}+k<0\end{cases} \\
m-\rho & = \begin{cases}m_{1}+m_{2}+k-\min \left(\rho_{i}\right) & m_{1}+k \geq 0 \\
\max \left(m_{2}-\rho_{2}, m_{1}+m_{2}+k-\rho_{1}+\epsilon\right) & m_{1}+k<0, m_{2}+k \geq 0 \\
\max \left(m_{i}-\rho_{i}\right) & m_{2}+k<0\end{cases}
\end{aligned}
$$

To apply the Taylor formula to products, we are now going to briefly study $\left[\mathfrak{e}_{j}(u)\right]_{\chi}$. Therefore recall that

$$
\left[\mathfrak{e}_{j}(u)\right]\left(x^{\prime \prime}, \eta^{\prime}\right)=\sum_{\nu} 2^{-\nu} \phi\left(2^{-\nu} \eta^{\prime}\right) \psi_{\nu}\left(D_{x^{\prime \prime}}\right) \partial_{x^{\prime}}^{j} u\left(x^{\prime \prime}, 0\right)
$$

And we obtain

$$
\begin{aligned}
{\left[\mathfrak{e}_{j}(u)\right]_{\chi}\left(x^{\prime \prime}, 0, \xi^{\prime}\right) } & =\int \chi\left(\eta^{\prime}, \xi^{\prime}\right) \sum_{\nu} 2^{-\nu} \phi\left(2^{-\nu} \eta^{\prime}\right) \psi_{\nu}\left(D_{x^{\prime \prime}}\right) \partial_{x^{\prime}}^{j} u\left(x^{\prime \prime}, 0\right) \mathrm{d} \eta^{\prime} \\
& =\left[\int \chi\left(\eta^{\prime}, \xi^{\prime}\right) \sum_{\nu} 2^{-\nu} \phi\left(2^{-\nu} \eta^{\prime}\right) \psi_{\nu}(\cdot) \mathrm{d} \eta^{\prime}\right]\left(D_{x^{\prime \prime}}\right) \partial_{x^{\prime}}^{j} u\left(x^{\prime \prime}, 0\right) \\
\Rightarrow \quad\left[\mathfrak{e}_{j}(u)\right]_{\chi}\left(x^{\prime \prime}, 0, \xi^{\prime}\right) & -\sum_{\nu} \psi_{\nu}\left(\xi^{\prime}\right) \varphi_{\nu}\left(D_{x^{\prime \prime}}\right) \partial_{x^{\prime}}^{j} u\left(x^{\prime \prime}, 0\right) \in S_{1,1}^{-m+\rho-j}
\end{aligned}
$$

So we have a decent understanding of the first approximation term for $\left[\mathfrak{e}_{j}(u)\right]_{\chi}$. With the following Proposition we will learn that in the Taylor expansion this is fair enough.

Proposition 2.26. Let $a\left(x^{\prime \prime}, \xi^{\prime}\right) \in S_{1,1}^{m, \rho}$ and $\alpha \in \mathbb{N}_{0}^{k}$ with $m+|\alpha|+k<0$ and

$$
\int\left(i \xi^{\prime}\right)^{\alpha} a\left(x^{\prime \prime}, \xi^{\prime}\right) d \xi^{\prime}=0
$$

then we obtain for $m-\rho \notin \mathbb{Z}$

$$
\partial_{x^{\prime}}^{\alpha} a_{\chi}\left(x^{\prime \prime}, 0, \xi^{\prime}\right) \in S_{1,1}^{m+k+|\alpha|, \rho}
$$


and for $m-\rho \in \mathbb{Z}$

$$
\partial_{x^{\prime}}^{\alpha} a_{\chi}\left(x^{\prime \prime}, 0, \xi^{\prime}\right) \in C_{*}^{\infty} S_{1,1}^{m+k+|\alpha|, \rho}
$$

ProOF.

$$
\begin{aligned}
& \left|\psi_{\nu}\left(D_{x^{\prime \prime}}\right) \partial_{\xi^{\prime}}^{\beta} \partial_{x^{\prime}}^{\alpha} a_{\chi}\left(x^{\prime \prime}, 0, \xi^{\prime}\right)\right|=\left|\psi_{\nu}\left(D_{x^{\prime \prime}}\right) \partial_{\xi^{\prime}}^{\beta} \int\left(i \zeta^{\prime}\right)^{\alpha} a\left(x^{\prime \prime}, \zeta^{\prime}\right) \chi\left(\zeta^{\prime}, \xi^{\prime}\right) \mathrm{d} \zeta^{\prime}\right| \\
& \lesssim \int_{\left\langle\zeta^{\prime}\right\rangle \gtrsim\left\langle\xi^{\prime}\right\rangle}\left\langle\zeta^{\prime}\right\rangle^{|\alpha|}\left|\psi_{\nu}\left(D_{x^{\prime \prime}}\right) a\left(x^{\prime \prime}, \zeta^{\prime}\right)\right|\left|\partial_{\xi^{\prime}}^{\beta}(\chi-1)\left(\zeta^{\prime}, \xi^{\prime}\right)\right| \mathrm{d} \zeta^{\prime} \\
& \lesssim \int_{\left\langle\zeta^{\prime}\right\rangle \gtrsim\left\langle\xi^{\prime}\right\rangle} 2^{-\nu \rho}\left\langle\zeta^{\prime}\right\rangle^{m+|\alpha|-|\beta|} \min \left(1,2^{-\nu}\left\langle\zeta^{\prime}\right\rangle\right)^{N} \mathrm{~d} \zeta^{\prime}
\end{aligned}
$$

Thus for $\left\langle\xi^{\prime}\right\rangle \geq 2^{\nu}$ we obtain as an estimate

$$
\left|\psi_{\nu}\left(D_{x^{\prime \prime}}\right) \partial_{\xi^{\prime}}^{\beta} \partial_{x^{\prime}}^{\alpha} a_{\chi}\left(x^{\prime \prime}, 0, \xi^{\prime}\right)\right| \lesssim 2^{-\nu \rho}\left\langle\xi^{\prime}\right\rangle^{m+k+|\alpha|-|\beta|}
$$

For $\left\langle\xi^{\prime}\right\rangle \leq 2^{\nu}$ we use the standard estimate

$$
\begin{aligned}
& \left|\psi_{\nu}\left(D_{x^{\prime \prime}}\right) \partial_{\xi^{\prime}}^{\beta} \partial_{x^{\prime}}^{\alpha} a_{\chi}\left(x^{\prime \prime}, 0, \xi^{\prime}\right)\right|=\left|\psi_{\nu}\left(D_{x^{\prime \prime}}\right) \partial_{\xi^{\prime}}^{\beta} \int\left(i \zeta^{\prime}\right)^{\alpha} a\left(x^{\prime \prime}, \zeta^{\prime}\right) \chi\left(\zeta^{\prime}, \xi^{\prime}\right) \mathrm{d} \zeta^{\prime}\right| \\
& \lesssim \int_{\left\langle\zeta^{\prime}\right\rangle \lesssim\left\langle\xi^{\prime}\right\rangle}\left\langle\zeta^{\prime}\right\rangle^{|\alpha|}\left|\psi_{\nu}\left(D_{x^{\prime \prime}}\right) a\left(x^{\prime \prime}, \zeta^{\prime}\right)\right|\left|\partial_{\xi^{\prime}}^{\beta}(\chi)\left(\zeta^{\prime}, \xi^{\prime}\right)\right| \mathrm{d} \zeta^{\prime} \\
& \lesssim \int_{\left\langle\zeta^{\prime}\right\rangle \lesssim\left\langle\xi^{\prime}\right\rangle} 2^{-\nu(\rho+N)}\left\langle\zeta^{\prime}\right\rangle^{m+|\alpha|+N}\left\langle\xi^{\prime}\right\rangle^{-|\beta|} \mathrm{d} \zeta^{\prime} \\
& \lesssim 2^{-\nu(\rho+N)}\left\langle\xi^{\prime}\right\rangle^{m+|\alpha|+N-|\beta|}
\end{aligned}
$$

For the estimates involving $\partial_{x^{\prime \prime}}^{\gamma}$ we use the same two ways to provide an estimate, taking the integral $\left\langle\zeta^{\prime}\right\rangle \lesssim\left\langle\xi^{\prime}\right\rangle$ if $m+|\alpha|+|\gamma|+k>0$ or the $\left\langle\zeta^{\prime}\right\rangle \gtrsim\left\langle\xi^{\prime}\right\rangle$ if $m+|\alpha|+$ $|\gamma|+k-|\beta|<0$. The argument fails if and only if both equal 0 , so indeed there is only one other estimate that fails.

This finishes the proof of Theorem 2.19

\section{Non-linear superposition}

In this section we will provide an approach to a theory of conormal distributions of type $(1,1)$ on manifolds by introducing transition functions under which the space of distributions is stable. Moreover the technical main theorem on nonlinear superposition 2.29 is going to be key in our study of the nonlinear propagation of conormality.

From this section onward we will focus on conormal distributions of codimension 1 , as they are the main field of interest for us and avoid some - mainly technical - difficulties one would encounter dealing with other dimensions. In particular a linearization like the later defined $F(x)$ is far more difficult to control in regularity and likewise its invertability in the case of higher codimension.

Definition 2.27 (diffeomorphism of conormal type). Let $m<-2$ and $\rho \geq 0$. Let $\kappa:\left(\mathbb{R}^{n}, \mathbb{R}^{n-1}\right) \rightarrow\left(\mathbb{R}^{n}, \mathbb{R}^{n-1}\right)$ be a diffeomorphism on $\mathbb{R}^{n}$ restricting to a diffeomorphism on $\mathbb{R}^{n-1}$ with decomposition

$$
\kappa=\kappa_{C}+\kappa_{G}+\kappa_{\infty}
$$


where $\kappa_{C}+\kappa_{G} \in I_{1,1}^{m, \rho}$ and $\kappa_{\infty} \in C^{\infty}$ such that $\nabla \kappa_{\infty} \in C_{b}^{\infty}$. If now further $(\nabla \kappa(x))^{-1} \in L^{\infty}\left(\mathbb{R}^{n} ; M_{n \times n}(\mathbb{R})\right)$ we will call $\kappa$ a diffeomorphism of conormal type and order $(m, \rho)$.

REMARK 2.28. As $\kappa\left(x^{\prime \prime}, 0\right)=\left(\kappa^{\prime \prime}\left(x^{\prime \prime}, 0\right), 0\right)$ we can with 2.17 and 2.18 obtain

$$
\kappa(x)=\left(\kappa^{\prime \prime}(x), x^{\prime} F(x)\right)
$$

with $F=F_{C}+F_{G}+F_{\infty}$, such that $F_{C} \in I_{1,1 ; \infty}^{m+1, \rho}$ fully symbolic, $F^{\infty} \in C_{b}^{\infty}$ and $F_{G} \in G^{m-\rho, 1}$.

The transformation of a conormal distribution under such a diffeomorphism of conormal type will still yield a conormal distribution.

TheOREM 2.29. Let $p \in I_{1,1}^{m_{1}, \rho_{1}}\left(\mathbb{R}^{n}, \mathbb{R}^{n-1}\right)$ and $\kappa$ be a local diffeomorphism of conormal type and order $\left(m_{2}, \rho_{2}\right)$ with $m_{1}<-1$ and $m_{2}<-2$, then we have

$$
p \circ \kappa \in I_{1,1}^{m, \rho}\left(\mathbb{R}^{n}, \mathbb{R}^{n-1}\right)
$$

With $m=\max \left(m_{1}, m_{2}\right)$ and

$$
m-\rho= \begin{cases}\max \left(m_{1}-\rho_{1}, m_{2}-\rho_{2}\right) & m_{1}+2<0 \\ \max \left(m_{1}-\rho_{1}, m_{2}-\rho_{2}+m_{1}+2\right) & m_{1}+2>0 \\ \max \left(m_{1}-\rho_{1}, m_{2}-\rho_{2}+\epsilon\right) & m_{1}+2=0\end{cases}
$$

Begin of Proof. We start by constructing the decomposition of $p \circ \kappa$ into parts we are going to investigate separately. Therefore we give a limiting structure for $p \circ \kappa$.

We have

$$
\kappa(x)=\left(\kappa^{\prime \prime}(x), F(x) x^{\prime}\right)
$$

Now we introduce a smoothed version of $\kappa$, note that $F=F_{G}+F_{C}+F_{\infty}$, with $F_{C} \in I_{1,1 ; \infty}^{m_{2}+1, \rho_{2}}$ fully symbolic, $F_{G} \in G^{m_{2}+1-\rho_{2}, 1}$ and $F_{\infty} \in C_{b}^{\infty}$. Correspondingly $\kappa^{\prime \prime}=\kappa_{C}^{\prime \prime}+\kappa_{G}^{\prime \prime}+\kappa_{\infty}^{\prime \prime}$.

$$
\begin{aligned}
& F_{s}(x)=\varphi\left(D_{x} / s\right) F_{G}(x)+\varphi\left(D_{x^{\prime}} / s\right) F_{C}(x)+F_{\infty}(x) \\
& \kappa_{s}^{\prime \prime}(x)=\varphi\left(D_{x} / s\right) \kappa_{G}^{\prime \prime}(x)+\varphi\left(D_{x^{\prime}} / s\right) \kappa_{C}^{\prime \prime}(x)+\kappa_{\infty}^{\prime \prime}(x) \\
& \kappa_{s}(x)=\left(\kappa_{s}^{\prime \prime}\left(x^{\prime \prime}\right), F_{s}(x) x^{\prime}\right)
\end{aligned}
$$

Further we give an analogue smoothing variant of $p$ in the decomposition $p=p_{G}+p_{C}$. Namely $p_{C, s}=\varphi\left(D_{x^{\prime}} / s\right) p_{C}$ and $p_{G, s}=\varphi(D / s) p_{G}$ and defining $p_{s}=p_{C, s}+p_{G, s}$. We obtain

$$
p \circ \kappa=\lim _{s \rightarrow \infty} p_{s} \circ \kappa_{s}=p_{t} \circ \kappa_{t}+\int_{t}^{\infty} \frac{\mathrm{d}}{\mathrm{d} s}\left(p_{s} \circ \kappa_{s}\right) \mathrm{d} s
$$

for $t$ chosen later. As $p_{t}$ and $\kappa_{t}$ are smooth, this component is of no interest in our analysis. We can further divide the components for the $\frac{\mathrm{d}}{\mathrm{d} s}$ parts obtaining

$$
\begin{aligned}
s \frac{\mathrm{d}}{\mathrm{d} s}\left(p_{s}\left(\kappa_{s}\right)\right)= & \left(\psi\left(D_{x^{\prime}} / s\right) p_{C}\right) \circ \kappa_{s}+\left(\psi\left(D_{x} / s\right) p_{G}\right) \circ \kappa_{s} \\
& +\left(\nabla p_{s}\right) \circ \kappa_{s} \cdot\left(\begin{array}{c}
\psi\left(D_{x^{\prime}} / s\right) \kappa_{C}^{\prime \prime}+\psi\left(D_{x} / s\right) \kappa_{G}^{\prime \prime} \\
x^{\prime} \psi\left(D_{x^{\prime}} / s\right) F_{C}+x^{\prime} \psi\left(D_{x} / s\right) F_{G}
\end{array}\right) .
\end{aligned}
$$


This results in the decomposition

$$
\begin{aligned}
& p \circ \kappa=p_{t} \circ \kappa_{t}+u_{\kappa, C}+u_{p, C}+u_{\kappa, G}+u_{p, G} \\
& u_{\kappa, C}=\int_{t}^{\infty}\left(\nabla p_{s}\right) \circ \kappa_{s} \cdot\left(\begin{array}{c}
\psi\left(D_{x^{\prime}} / s\right) \kappa_{C}^{\prime \prime} \\
x^{\prime} \psi\left(D_{x^{\prime}} / s\right) F_{C}
\end{array}\right) \mathrm{d} \ln (s) \\
& u_{p, C}=\int_{t}^{\infty}\left(\psi\left(D_{x^{\prime}} / s\right) p_{C}\right) \circ \kappa_{s} \mathrm{~d} \ln (s) \\
& u_{\kappa, G}=\int_{t}^{\infty}\left(\nabla p_{s}\right) \circ \kappa_{s} \cdot\left(\begin{array}{c}
\psi\left(D_{x} / s\right) \kappa_{G}^{\prime \prime} \\
x^{\prime} \psi\left(D_{x} / s\right) F_{G}
\end{array}\right) \mathrm{d} \ln (s) \\
& u_{p, G}=\int_{t}^{\infty}\left(\psi\left(D_{x} / s\right) p_{G}\right) \circ \kappa_{s} \mathrm{~d} \ln (s)
\end{aligned}
$$

The proof of the embedding results for each of the components of (5) is transfered into the subsequent Propositions 2.33 2.34, 2.35 and 2.38.

As similar results will hold for transformations fulfilling the essential estimates of diffeomorphisms of conormal type, we give the following definition.

Definition 2.30. Let $\left(\kappa_{t}\right)=\left(\kappa_{t}^{\prime \prime}, x^{\prime} F_{t}\right)_{t \geq 1}$ be a smoothing family of a diffeomorphism $\kappa$. The $(m, \rho)$ essential bounds for $\kappa_{t}$ are

$$
\begin{aligned}
\left\|\partial_{x^{\prime}}^{\alpha^{\prime}} \nabla_{x^{\prime \prime}} \kappa_{t}\left(\cdot, x^{\prime}\right)\right\|_{C_{*}^{-m-2+\rho}} & \leq C_{1} t^{\left|\alpha^{\prime}\right|} \\
\left\|\partial_{x^{\prime}}^{1+\alpha^{\prime}} \kappa_{t}\left(\cdot, x^{\prime}\right)\right\|_{C_{*}^{-m-1+\rho}} & \leq C_{2} t^{1+\left|\alpha^{\prime}\right|} \\
\left\|\nabla\left(\kappa^{-1}\right)\right\|_{L^{\infty}} & \leq C_{3} .
\end{aligned}
$$

And a uniform lower bound on the gradient for large enough times

$$
\inf \left\{t^{\prime} \geq 1 ; \sup _{t \geq t^{\prime}}\left\|\nabla\left(\kappa_{t}^{-1}\right)\right\|_{L^{\infty}} \leq 2\left\|\nabla\left(\kappa^{-1}\right)\right\|_{L^{\infty}}\right\} \leq T .
$$

The collection $\left(C_{1}, C_{2}, C_{3}, T\right)$ we denote as $\|\kappa\|_{\text {ess }}$.

REMARK 2.31. $T$ is dominated by a function of $C_{3}$ and $\left\|\kappa-\kappa_{\infty}\right\|_{C_{*}^{-m-1}}$. As

$$
\begin{aligned}
\left\|\kappa-\kappa_{t}\right\| & \lesssim\left\|\kappa-\kappa_{\infty}\right\|_{C_{*}^{-m-1}} t^{m+1} \\
\left\|\nabla\left(\kappa-\kappa_{t}\right)\right\| & \lesssim\left\|\nabla\left(\kappa-\kappa_{\infty}\right)\right\|_{C_{*}^{-m-2}} t^{m+2}
\end{aligned}
$$

and $C_{3}$ gives a bound $\|\delta \kappa\|_{C^{1}}<c$ such that $\kappa+\delta \kappa$ is still a diffeomorphism globally. Then estimating the perturbation of $(\nabla(\kappa+\delta \kappa))^{-1}$ - again using $C_{3}$ - gives $c \geq c^{\prime}>0$ such that $\left|(\nabla(\kappa+\delta \kappa))^{-1}\right| \leq 2 C_{3}$ for all $\|\delta \kappa\|_{C^{1}}<c_{1}$, which gives $T\left(c_{1}\right)$.

First we provide a general technical result resolving the sharp similarities of all but the first two terms within the integral.

LEMMA 2.32. Let $\kappa_{s}$ be a smoothing family of a local diffeomorphism of conormal type of order $\left(m_{2}, \rho_{2}\right)$ constructed from (1) and (3). Let further $a \in I_{1,1}^{m_{1}+1, \rho_{1}}$, given with smoothed decomposition $a_{s}=\varphi\left(D_{x^{\prime}} / s\right) a_{C}+\varphi\left(D_{x} / s\right) a_{G}$, then the symbol

$$
q(x, \xi)=\int_{t}^{\infty} a_{s} \circ \kappa_{s} \psi(\xi / s) \mathrm{d} \ln (s)
$$


fulfills for $\rho^{\prime}=\min \left(-m_{1}-2,-m_{2}-1\right)$ and $\rho^{\prime}+\rho^{\prime \prime}=\min \left(-m_{1}-2+\rho_{1},-m_{2}-1+\rho_{2}\right)$

$$
q(x, \xi) \in S_{1,1}^{\left(\rho^{\prime}\right)_{\epsilon}^{-},\left(\rho^{\prime}\right)_{\epsilon}^{+}, \rho^{\prime \prime}} .
$$

If $\kappa_{s}$ only has the essential bounds $C_{1}, C_{2}$, then

$$
q(x, \xi) \in S_{1,1}^{\left(\rho^{\prime}\right)_{\epsilon}^{-}, 0, \rho^{\prime \prime}+\left(\rho^{\prime}\right)_{\epsilon}^{+}}
$$

with each seminorm bounded by a product of some constant depending on $C_{1}, C_{2}$ and a seminorm of $a$.

Proof. We have to show $\forall\left|\alpha^{\prime \prime}\right| \neq \rho^{\prime \prime}+\left(\rho^{\prime}-\left|\alpha^{\prime}\right|\right)^{+},\left|\alpha^{\prime}\right| \neq \rho^{\prime}$

$$
\begin{aligned}
\left|\partial_{x^{\prime}}^{\alpha^{\prime}} \partial_{x^{\prime \prime}}^{\alpha^{\prime \prime}} \partial_{\xi}^{\beta} q(x, \xi)\right| & \lesssim\langle\xi\rangle\rangle^{\left(\left|\alpha^{\prime}\right|-\rho^{\prime}+\left(\left|\alpha^{\prime \prime}\right|-\rho^{\prime \prime}\right)^{+}\right)^{+}-|\beta|} \\
\left\|\partial_{x^{\prime}}^{\alpha^{\prime}} \partial_{\xi}^{\beta} q\left(\cdot, x^{\prime}, \xi\right)\right\|_{C_{*}^{\rho^{\prime \prime}+\left(\rho^{\prime}-\left|\alpha^{\prime}\right|\right)^{+}}} & \lesssim\langle\xi\rangle^{\left(\left|\alpha^{\prime}\right|-\rho^{\prime}\right)^{+-|\beta|}}
\end{aligned}
$$

and for $\left|\alpha^{\prime}\right|=\rho^{\prime}$

$$
\begin{aligned}
\left|\partial_{x^{\prime}}^{\alpha^{\prime}} \partial_{x^{\prime \prime}}^{\alpha^{\prime \prime}} \partial_{\xi}^{\beta} q(x, \xi)\right| & \lesssim\langle\xi\rangle^{\epsilon+\left(\left|\alpha^{\prime \prime}\right|-\rho^{\prime \prime}\right)^{+-}-|\beta|} \\
\left\|\partial_{x^{\prime}}^{\alpha^{\prime}} \partial_{\xi}^{\beta} q\left(\cdot, x^{\prime}, \xi\right)\right\|_{C_{*}^{\rho^{\prime \prime}+\epsilon}} & \lesssim\langle\xi\rangle^{\epsilon-|\beta|}
\end{aligned}
$$

At first, as $|\xi| \simeq s$ on the support of $\psi(\xi / s)$, thus growth estimates are to be taken with respect to $s$ and it is sufficient to show the estimates for $\beta=0$. Using a generalization of Faà di Bruno's formula, the derivative $\partial_{x}^{\alpha}\left(a_{s} \circ \kappa_{s}\right)(x)$ (ignoring combinatorial factors and the necessity to choose the correct components of $\kappa_{s}^{\prime \prime}$ ) has the form

$$
\sum\left(\partial_{x^{\prime \prime}}^{d_{1}} \partial_{x^{\prime}}^{d_{2}+d_{3}} a_{s}\right)\left(\kappa_{s}(x)\right)\left(x^{\prime}\right)^{d_{2}-c} \prod_{i=1}^{\left|d_{1}\right|} \partial_{x}^{\beta_{i}^{\prime \prime}} \kappa_{s}^{\prime \prime}(x) \prod_{j=1}^{d_{2}} \partial_{x}^{\gamma_{j}} F_{s}(x) \prod_{\ell=1}^{d_{3}} \partial_{x}^{\delta_{\ell}} F_{s}(x)
$$

With the conditions for the sum being $\sum_{i=1}^{\left|d_{1}\right|} \beta_{i}+\sum_{j=1}^{d_{2}} \gamma_{j}+\sum_{\ell=1}^{d_{3}} \delta_{\ell}+\left(c+d_{3}\right) e_{x^{\prime}}=$ $\alpha$, where $e_{x^{\prime}}$ is the unit vector corresponding to the $x^{\prime}$ coordinate, and $c \leq d_{2}$, $\left|\beta_{i}\right|,\left|\gamma_{j}\right| \geq 1,\left|\delta_{\ell}\right| \geq 0$.

Thus, as $x^{\prime} F_{s}\left(x^{\prime \prime}\right)$ and $\kappa_{s}^{\prime \prime}$ are uniformly bounded in $C^{1}$, taking a higher derivative on the outer function does not increase $s$ growth from factors of derivatives of the inner functions. Likewise as raising the $x^{\prime}$-derivative of the outer function with a $x^{\prime \prime}$-derivative on $F_{s}$ does only change the underlying $x$-regularity from $a_{G}$ and not the principal $x^{\prime}$-regularity from $a_{C}$ due to the factor $x^{\prime}$ from chain rule. Overall we obtain the maximum $s$ growth, from the summands, where all derivatives are effectively acting either on $\kappa_{s}$ or on $a_{s}$

$$
\begin{aligned}
& \left|\partial_{x^{\prime \prime}}^{\alpha^{\prime \prime}} \partial_{x^{\prime}}^{\alpha^{\prime}} \partial_{\xi}^{\beta} q(x, \xi)\right| \\
& \lesssim \sum_{\alpha=a_{1}+a_{2}} \int_{|\xi| / 2}^{2|\xi|} s^{\left(m_{1}+2+\left|a_{1}^{\prime}\right|+\left(\left|a_{1}^{\prime \prime}\right|-\rho_{1}\right)_{\epsilon}^{+}\right)_{\epsilon}^{+}} s^{\left(m_{2}+1+\left|a_{2}^{\prime}\right|+\left(\left|a_{2}^{\prime \prime}\right|-\rho_{2}\right)_{\epsilon}^{+}\right)_{\epsilon}^{+}} s^{-|\beta|} \mathrm{d} \ln (s) \\
& \lesssim\langle\xi\rangle^{\left(\left|\alpha^{\prime}\right|-\rho^{\prime}+\left(\left|\alpha^{\prime \prime}\right|-\rho^{\prime \prime}\right)_{\epsilon}^{+}\right)_{\epsilon}^{+}-|\beta|} .
\end{aligned}
$$

To obtain the Hölder-Zygmund norm, take the decomposition of $a=a_{C}+a_{G}$. Take $F$ to be a smooth variable with $F \geq \delta>0$, then $a_{C, s}\left(x^{\prime} F, \kappa_{s}^{\prime \prime}(x)\right)$ is smooth in $F$ due 
to the $x^{\prime}$ factor, with derivatives uniformly bounded by $s^{\left(m_{1}+2\right)_{\epsilon}^{+}}$given $\delta>0$ fixed. Therefore

$$
\left\|a_{C, s}\left(x^{\prime} F_{s}\left(x^{\prime}, \cdot\right), \kappa_{s}^{\prime \prime}(x)\right)\right\|_{C_{*}^{-m_{2}-2+\rho_{2}}} \lesssim s^{\left(m_{1}+2\right)_{\epsilon}^{+}}
$$

but also its derivative fulfills, as $F \in I_{1,1 ; 1}^{m_{1}+1, \rho_{1}}$,

$$
\left\|x^{\prime} \partial_{x^{\prime \prime}} F_{s}\left(x^{\prime}, \cdot\right)\left(\partial_{x^{\prime}} a_{C, s}\right)\left(x^{\prime} F_{s}\left(x^{\prime}, \cdot\right), \kappa_{s}^{\prime \prime}(x)\right)\right\|_{C_{*}^{-m_{2}-2+\rho_{2}}} \lesssim s^{\left(m_{1}+2\right)_{\epsilon}^{+}}
$$

yielding

$$
\left\|a_{C, s}\left(x^{\prime} F_{s}\left(x^{\prime}, \cdot\right), \kappa_{s}^{\prime \prime}(x)\right)\right\|_{C_{*}^{-m_{2}-1+\rho_{2}}} \lesssim s^{\left(m_{1}+2\right)_{\epsilon}^{+}} .
$$

Now we have $\kappa^{\prime \prime}\left(x^{\prime}, \cdot\right) \in C_{*}^{-m_{2}-1+\rho_{2}}$ and $\left\|a_{C, s}\left(x^{\prime}, \cdot\right)\right\|_{C^{\left(-m_{1}-2\right)_{\epsilon}^{+}+\rho_{1}}} \lesssim s^{\left(m_{1}+2\right)_{\epsilon}^{+}}$, which yields

$$
\left\|a_{C, s}\left(x^{\prime} F_{s}\left(x^{\prime}, y^{\prime \prime}\right), \kappa_{s}^{\prime \prime}\left(x^{\prime}, \cdot\right)\right)\right\|_{C_{*}^{\min }} \lesssim s^{\left(m_{1}+2\right)_{\epsilon}^{+}},
$$

where $\min =\min \left(-m_{2}-1+\rho_{2},\left(-m_{1}-2\right)_{\epsilon}^{+}+\rho_{1}\right)$. Now taking the trace $y^{\prime \prime}=x^{\prime \prime}$ yields the sufficient estimate on $a_{C, s} \circ \kappa_{s}$. For $a_{G, s}$ the computation does not need a differentiation between the $x^{\prime}$ and $x^{\prime \prime}$ component within $a_{s}$ and works just as the $\kappa^{\prime \prime}\left(x^{\prime}, x^{\prime \prime}\right)$ case.

Taking $x^{\prime}$ derivatives, as (6) shows, does only add some $d_{2}+d_{3}$ on $m_{1}$, subtracts $\left|d_{1}\right|$ from $\rho_{1}$ and yields a factor $\left(x^{\prime}\right)^{d_{2}-c}$ together with some inner derivatives. Thus by paramultiplication this case is immediate from the $a_{s} \circ \kappa_{s}$ result.

The proof for the essential estimates requires the very same computations just with the adapted initial estimates.

From this we will immediately obtain the (singular) contribution of $\kappa$ to the composition via multiplication of symbols.

Proposition 2.33. Let $p \in I_{1,1}^{m_{1}, \rho_{1}}\left(\mathbb{R}^{n}, \mathbb{R}^{n-1}\right)$ and $\kappa_{s}$ be a smoothing family of a local diffeomorphism of conormal type of order $\left(m_{2}, \rho_{2}\right)$ constructed from (1) and (3) with $m_{2}<-2$, then for the following term from (5), we obtain

$$
\int_{t}^{\infty} x^{\prime}\left(\nabla p_{s}\right) \circ \kappa_{s} \cdot \psi\left(D_{x^{\prime}} / s\right) F_{C} \mathrm{~d} \ln (s) \in \begin{cases}I_{1,1}^{m_{2}, \min \left(\rho_{2},-m_{1}-2+\rho_{1}\right)} & m_{1}+2<0 \\ I_{1,1}^{m_{2}+m_{1}+2, \min \left(\rho_{1}, \rho_{2}\right)} & m_{1}+2>0 \\ I_{1,1}^{m_{2}+\epsilon, \min \left(\rho_{1}, \rho_{2}\right)+\epsilon} & m_{1}+2=0\end{cases}
$$

Proof. Let $p_{C}$ have the symbol $a\left(x^{\prime \prime}, \eta^{\prime}\right)$, then the operator with symbol

$$
q(x, \eta)=\int_{t}^{\infty}\left(\nabla p_{s}\right) \circ \kappa_{s} \cdot \psi\left(\eta^{\prime} / s\right) \mathrm{d} \ln (s)
$$

acts on $p_{C}$ via a product on the symbol level. Via Lemma 2.32 this yields a symbol of anisotropic improved smoothness. Using Proposition 2.12 and combining with the $x^{\prime}$ factor, we obtain the claim.

The singular contribution of $p$ will now be obtained by an integral of the usual transformation result for $p$ under transformations. There is just a cutoff present and the transformation used changes depending on the scale parameter. 
Proposition 2.34. Let $p \in I_{1,1}^{m_{1}, \rho_{1}}\left(\mathbb{R}^{n}, \mathbb{R}^{n-1}\right)$ and $\kappa$ be a local diffeomorphism of conormal type and order $\left(m_{2}, \rho_{2}\right)$ with $m_{2}<-2$, then for the respective component in (5), we obtain

$$
\begin{array}{r}
\int_{t}^{\infty}\left(\psi(D / s) p_{C}\right) \circ \kappa_{s} \mathrm{~d} \ln (s)=\int e^{i x^{\prime} \zeta^{\prime}} c\left(x, \zeta^{\prime}\right) d \zeta^{\prime} \\
\in\left(x, \zeta^{\prime}\right) \in I_{1,1}^{m_{1}, \rho^{\prime}+\rho^{\prime \prime}} \\
\in S_{1,1}^{m_{1}, \rho^{\prime}, \rho^{\prime \prime}}
\end{array}
$$

with $\rho^{\prime}=\min \left(\rho_{1},-m_{2}-2\right)$ and $\rho^{\prime}+\rho^{\prime \prime}=\min \left(\rho_{1},-m_{2}-2+\rho_{2}\right)$.

If $\kappa_{s}$ only has the essential bounds $C_{1}, C_{2}$, then

$$
c\left(x, \zeta^{\prime}\right) \in S_{1,1}^{m_{1}, 0, \rho^{\prime}+\rho^{\prime \prime}}
$$

with each seminorm bounded by a product of some constant depending on $C_{1}, C_{2}$ and a seminorm of $p$.

Proof. Observe the integral in the symbol representation with $a$ being the symbol of $p_{C}$.

$$
\begin{aligned}
& \int_{t}^{\infty} \int e^{i x^{\prime} F_{s}^{\prime} \eta^{\prime}} a\left(\kappa_{s}(x), \eta^{\prime}\right) \psi\left(\eta^{\prime} / s\right) \mathrm{d} \eta^{\prime} \mathrm{d} \ln (s) \\
& =\int e^{i x^{\prime} \zeta^{\prime}} \int_{t}^{\infty} a\left(\kappa_{s},\left(F_{s}^{\prime}\right)^{-1} \zeta^{\prime}\right)\left(F_{s}^{\prime}\right)^{-1} \psi\left(\left(F_{s}^{\prime}\right)^{-1} \zeta^{\prime} / s\right) \mathrm{d} \ln (s) \mathrm{d} \zeta^{\prime}
\end{aligned}
$$

Now we can estimate the resulting symbol

$$
c\left(x, \zeta^{\prime}\right)=\int_{t}^{\infty} a\left(\kappa_{s},\left(F_{s}^{\prime}\right)^{-1} \zeta^{\prime}\right)\left(F_{s}^{\prime}\right)^{-1} \psi\left(\left(F_{s}^{\prime}\right)^{-1} \zeta^{\prime} / s\right) \mathrm{d} \ln (s)
$$

by considering

$$
\begin{aligned}
& \left|\partial_{x^{\prime \prime}}^{\alpha^{\prime \prime}} \partial_{x^{\prime}}^{\alpha^{\prime}} \partial_{\zeta^{\prime}}^{\beta} a\left(\kappa_{s},\left(F_{s}^{\prime}\right)^{-1} \zeta^{\prime}\right)\left(F_{s}^{\prime}\right)^{-1} \psi\left(\left(F_{s}^{\prime}\right)^{-1} \zeta^{\prime} / s\right)\right| \\
& \lesssim \sum_{\substack{\alpha=\alpha_{1}+\alpha_{2} \\
\beta=\beta_{1}+\beta_{2}}}\left\langle\zeta^{\prime}\right\rangle^{m_{1}-\left|\beta_{1}\right|+\left(\left|\alpha_{1}\right|-\rho_{1}\right)_{\epsilon}^{+}} s^{\left(m_{2}+2+\left|\alpha_{2}^{\prime}\right|+\left(\left|\alpha_{1}^{\prime \prime}\right|-\rho_{2}\right)_{\epsilon}^{+}\right)_{\epsilon}^{+}-\left|\beta_{2}\right|}
\end{aligned}
$$

Now taking into account, that $s \simeq\left\langle\zeta^{\prime}\right\rangle$, we obtain taking the integral

$$
\begin{aligned}
& \left|\partial_{x^{\prime \prime}}^{\alpha^{\prime \prime}} \partial_{x^{\prime}}^{\alpha^{\prime}} \partial_{\zeta^{\prime}}^{\beta} c\left(x, \zeta^{\prime}\right)\right| \\
& \lesssim \int_{\left|\zeta^{\prime}\right| / 2}^{2\left|\zeta^{\prime}\right|} \sum_{\substack{\alpha=\alpha_{1}+\alpha_{2} \\
\beta=\beta_{1}+\beta_{2}}}\left\langle\zeta^{\prime}\right\rangle^{m_{1}-\left|\beta_{1}\right|+\left(\left|\alpha_{1}\right|-\rho_{1}\right)_{\epsilon}^{+}} s^{\left(m_{2}+2+\left|\alpha_{2}^{\prime}\right|+\left(\left|\alpha_{1}^{\prime \prime}\right|-\rho_{2}\right)_{\epsilon}^{+}\right)_{\epsilon}^{+}-\left|\beta_{2}\right|} \mathrm{d} \ln (s) \\
& \lesssim\left\langle\zeta^{\prime}\right\rangle^{m_{1}-|\beta|+\left(\left|\alpha^{\prime}\right|+\left(\left|\alpha^{\prime \prime}\right|-\rho^{\prime \prime}\right)_{\epsilon}^{+}-\rho^{\prime}\right)_{\epsilon}^{+}}
\end{aligned}
$$

With $\rho^{\prime}+\rho^{\prime \prime}=\min \left(\rho_{1},-m_{2}+\rho_{2}-2\right)$ and $\rho^{\prime}=\min \left(\rho_{1},-m_{2}-2\right)$. Again the Hölder-Zygmund norms are obtained via composition estimates. Observe that both $a\left(\kappa_{s}^{\prime \prime}\left(x^{\prime}, x^{\prime \prime}\right), F \zeta^{\prime}\right)$ and $\psi\left(F \zeta^{\prime} / s\right)$ are uniformly smooth in $F$. So with the same arguments as in the proof of Proposition 2.33, we obtain the claim.

Two of the remainder summands can easily be estimated using the mapping properties from the previous section.

Proposition 2.35. Let $p \in I_{1,1}^{m_{1}, \rho_{1}}\left(\mathbb{R}^{n}, \mathbb{R}^{n-1}\right)$ and $\kappa$ be a local diffeomorphism of conormal type and order $\left(m_{2}, \rho_{2}\right)$ with $m_{2}<-2$, then for the following terms in 
(5), we obtain

$$
\begin{array}{r}
\int_{t}^{\infty}\left(\nabla_{x^{\prime \prime}} p_{s}\right) \circ \kappa_{s} \cdot \psi\left(D_{x} / s\right) \kappa_{G}^{\prime \prime}+x^{\prime}\left(\partial_{x^{\prime}} p_{s}\right) \circ \kappa_{s} \cdot \psi\left(D_{x} / s\right) F_{G} \mathrm{~d} \ln (s) \\
\in G^{\left(-m_{1}-2\right)_{\epsilon}^{-}+m_{2}-\rho_{2}}
\end{array}
$$

Proof. Use Lemma 2.32 to obtain

$$
q(x, \xi)=\int_{t}^{\infty}\left(\nabla_{x} p_{s}\right) \circ \kappa_{s} \cdot \psi(\xi / s) \mathrm{d} \ln (s) \in S_{1,1}^{\left(\rho^{\prime}\right)_{\epsilon}^{-},\left(\rho^{\prime}\right)_{\epsilon}^{+}, \rho^{\prime \prime}}
$$

and using Lemma 2.13, we obtain

$$
q^{\prime \prime}\left(x, D_{x}\right) \kappa_{G}^{\prime \prime} \in G^{\left(\rho^{\prime}\right)_{\epsilon}^{-}+m_{2}-\rho_{2}}
$$

Likewise, we obtain

$$
x^{\prime} q^{\prime}(x, D) F_{G}=\left[x^{\prime}, q^{\prime}(x, D)\right] F_{G}+q^{\prime}(x, D) x^{\prime} F_{G} \in G^{\left(\rho^{\prime}\right)_{\epsilon}^{-}+m_{2}-\rho_{2}}
$$

Now if $\left(\rho^{\prime}\right)_{\epsilon}^{-}>0$, then $\rho^{\prime}=-m_{1}-2$, which yields the claim.

As the estimate for the last remainder summand is a bit lengthy and will repeat in later proofs, we will give a major part of the required estimate as a separate Lemma. In order for the reader not to lose track of what we are going to establish, the Lemma essentially says that given a function with compact spectrum and taking a nonlinear superposition with a function with compact spectrum will yield a function with improved decay at high frequencies. The improvement will be of order $r-1>0$. This is the important part, as the low frequency behavior is determined already without the nonlinear superposition, given the superposition function is uniformly bounded in $C_{*}^{r}$.

Lemma 2.36. Let $\left(u_{s}\right)_{s \geq t} \in C^{\infty}\left(\mathbb{R}^{n} ; \mathbb{R}^{d}\right)$ and $\left(c_{s}\right)_{s \geq t} \in C^{\infty}\left(\mathbb{R}^{d}\right)$ be two families of smooth functions satisfying for some $r>1$

$$
\begin{aligned}
&\left|\partial_{x}^{\alpha} u_{s}\right| \lesssim \begin{cases}1 & 1 \leq|\alpha|<r \\
s^{|\alpha|-r} & |\alpha|>r\end{cases} \\
&\left|\partial_{x}^{\alpha} c_{s}\right| \lesssim \begin{cases}1 & |\alpha|<r-1 \\
s^{|\alpha|-r+1} & |\alpha|>r-1\end{cases}
\end{aligned}
$$

and let $\phi \in C_{c}^{\infty}\left(\mathbb{R}^{d}\right)$ with $\operatorname{supp} \phi \subseteq B_{R}(0)$, then we can define the linear operators $T_{s}: \mathcal{S}\left(\mathbb{R}^{d}\right) \rightarrow \mathcal{S}^{\prime}\left(\mathbb{R}^{n}\right)$ with kernels $K_{s}(x, y)$ via

$$
T_{s}(f)(x)=\psi_{\mu}(D)\left[\left(c_{s} \phi(D / s) f\right) \circ u_{s}\right](x)=\int K_{s}(x, y) f(y) \mathrm{d} y .
$$

For every $\ell \geq 0$ and for $2^{\mu} \gtrsim \sup _{s}\left|\nabla u_{s}\right| R s$ we have the estimate

$$
\left\|K_{s}\left(x, u_{s}(x)+y\right)\right\|_{L^{1}\left(\mathbb{R}_{y}^{d} ; L^{\infty}\left(\mathbb{R}_{x}^{n}\right)\right)} \lesssim 2^{-\mu(r-1)}\left(s 2^{-\mu}\right)^{\ell} .
$$

Proof. We introduce $2^{\nu}=s$ and $\phi_{\nu}(\xi)=\phi\left(2^{-\nu} \xi\right)$ for simplicity of notation. We have the equation for the kernel

$$
K(x, y)=\int e^{i\left(y-u_{s}(z)\right) \xi} e^{i(z-x) \eta} c_{s}(z) \phi_{\nu}(\xi) \psi_{\mu}(\eta) \mathrm{d} \xi \mathrm{d} z d \eta .
$$

In the following, we will use partial integration in combination with estimates on the respective phase factor. In these estimates we will do our computations as if in the one-dimensional case. For general dimensions, if one decomposes the integral into 
sectors with dominant coordinate component for the phase factor and uses partial integration with this component on this sector and then recombines, one reobtains the result. Therefore we will pretend to have a one-dimensional case for simplicity of notation.

Note that $\left|\eta-\partial_{z} u_{s}(z) \xi\right| \gtrsim 2^{\mu}$ on the support of $\phi_{\nu}(\xi) \psi_{\mu}(\eta)$ due to $2^{\mu} \gtrsim \sup _{t}\left|\nabla u_{s}\right| R s$. Now as $\frac{-i}{\eta-\partial_{z} u_{s}(z) \xi} \partial_{z} e^{i\left(z \eta-u_{s}(z) \xi\right)}=e^{i\left(z \eta-u_{s}(z) \xi\right)}$ we obtain using partial integration

$$
K(x, y)=\int e^{i\left(y-u_{s}(z)\right) \xi} e^{i(z-x) \eta}\left[\partial_{z} \frac{i}{\eta-\partial_{z} u_{s}(z) \xi}\right]^{N} c_{s}(z) \phi_{\nu}(\xi) \psi_{\mu}(\eta) \mathrm{d} \xi \mathrm{d} z \mathrm{~d} \eta .
$$

Now we decompose the integral into four regions for $i, j \in\{ \pm\}$

$$
\begin{array}{lr}
A^{i, j}=B^{i} \cap C^{j} & \\
B^{+}=\left\{z \in \mathbb{R}^{n} ;|z-x|<\delta_{1}\right\} & B^{-}=\left\{z \in \mathbb{R}^{n} ;|z-x| \geq \delta_{1}\right\} \\
C^{+}=\left\{z \in \mathbb{R}^{n} ;\left|y-u_{s}(z)\right|<\delta_{2}\right\} & C^{-}=\left\{z \in \mathbb{R}^{n} ;\left|y-u_{s}(z)\right| \geq \delta_{2}\right\} .
\end{array}
$$

Thus we have

$$
K(x, y)=\sum_{i, j \in \pm} \int_{A^{i, j}} e^{i\left(y-u_{s}(z)\right) \xi} e^{i(z-x) \eta}\left[\partial_{z} \frac{i}{\eta-\partial_{z} u_{s}(z) \xi}\right]^{N} c_{s}(z) \phi_{\nu}(\xi) \psi_{\mu}(\eta) \mathrm{d} \xi \mathrm{d} z \mathrm{~d} \eta .
$$

Now we apply other partial integrations depending on $i, j$ with $L^{-}=M^{-}=0$ and $L^{+}=M^{+}=N \gg 0$ and obtain

$$
\begin{gathered}
K(x, y)=\sum_{i, j} \int_{A^{i, j}} e^{i\left(y-u_{s}(z)\right) \xi} e^{i(z-x) \eta}\left[\partial_{\eta} \frac{i}{z-x}\right]^{L^{i}}\left[\partial_{\xi} \frac{i}{y-u_{s}(z)}\right]^{M^{j}} \\
{\left[\partial_{z} \frac{i}{\eta-\partial_{z} u_{s}(z) \xi}\right]^{N} c_{s}(z) \phi_{\nu}(\xi) \psi_{\mu}(\eta) \mathrm{d} \xi \mathrm{d} z \mathrm{~d} \eta .}
\end{gathered}
$$

We will estimate the absolute value of $K\left(x, u_{s}(x)+\tilde{y}\right)$ via

$$
\begin{aligned}
\left|K\left(x, u_{s}(x)+\tilde{y}\right)\right| \leq \sum_{i, j} \int_{A^{i, j}}|z-x|^{-L^{i}}\left|\tilde{y}+u_{s}(x)-u_{s}(z)\right|^{-M^{j}} \times \\
\\
\qquad \partial_{\eta}^{L^{i}} \partial_{\xi}^{M^{j}}\left[\partial_{z} \frac{i}{\eta-\partial_{z} u_{s}(z) \xi}\right]^{N} c_{s}(z) \phi_{\nu}(\xi) \psi_{\mu}(\eta) \mid \mathrm{d} \xi \mathrm{d} z \mathrm{~d} \eta \mathrm{d} y .
\end{aligned}
$$

Now the $\partial_{\eta}$ and $\partial_{\xi}$ derivatives acting on $\phi_{\nu}(\xi) \psi_{\mu}(\eta)$ yield a factor of $2^{-\mu}$ and $2^{-\nu}$ respectively for each derivative taken. So it remains to estimate on the support of $\phi_{\nu}(\xi) \psi_{\mu}(\eta)$

$$
\begin{aligned}
& \left|\partial_{\eta}^{L} \partial_{\xi}^{M}\left[\partial_{z} \frac{i}{\eta-\partial_{z} u_{s}(z) \xi}\right]^{N}\right|
\end{aligned}
$$

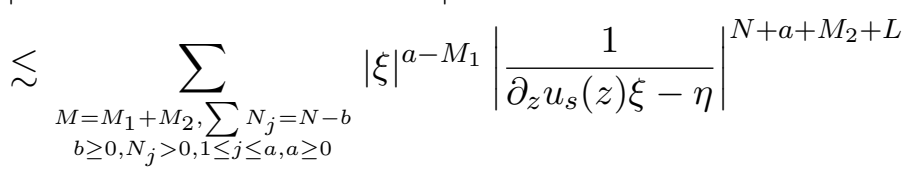

$$
\begin{aligned}
& \left|\partial_{z} u_{s}(z)\right|^{M_{2}} \prod_{j=1}^{a}\left|\partial_{z}^{N_{j}+1} u_{s}(z)\right|\left|\partial_{z}^{b} c_{s}(z)\right| \\
& \lesssim \sum 2^{\left(a-M_{1}\right) \nu} 2^{-\mu\left(N+a+M_{2}+L\right)} 2^{\nu(N-b-a+2-r)^{+}} 2^{\nu(b-r+1)^{+}} \\
& \lesssim 2^{-\nu(M+r-1)} 2^{-\mu L} 2^{-(\mu-\nu) N} \text {. }
\end{aligned}
$$


Now we may choose $\delta_{1}=2^{-\mu}$ and $\delta_{2}=2^{-\nu}$. So applying (7) to this yields

$$
\begin{aligned}
& \left|K\left(x, u_{s}(x)+\tilde{y}\right)\right| \\
& \lesssim 2^{-\nu(r-1)-(\mu-\nu)(N+1)} \int_{\substack{|\xi| \simeq 2^{\nu} \\
|\eta| \simeq 2^{\mu}}}\left\langle 2^{\mu}(z-x)\right\rangle^{-L}\left\langle 2^{\nu}\left(\tilde{y}+u_{s}(x)-u_{s}(z)\right)\right\rangle^{-M} \mathrm{~d} \eta \mathrm{d} z \mathrm{~d} \xi .
\end{aligned}
$$

Now as $\langle a\rangle^{-1}\langle a+b\rangle^{-1} \lesssim\langle b\rangle^{-1}$ and as $2^{\mu} \gtrsim 2^{\nu}$ and $\left|u_{s}(z)-u_{s}(x)\right| \lesssim|z-x|$, we obtain

$$
\begin{aligned}
& \left|K\left(x, u_{s}(x)+\tilde{y}\right)\right| \\
& \lesssim 2^{-\nu(r-1)-(\mu-\nu)(N+1)} \int_{\substack{|\xi| \simeq 2^{\nu} \\
|\eta| \simeq 2^{\mu}}}\left\langle 2^{\nu} \tilde{y}\right\rangle^{-M}\left\langle 2^{\mu}(z-x)\right\rangle^{-(L-M)} \mathrm{d} \eta \mathrm{d} z \mathrm{~d} \xi \\
& \lesssim 2^{-\nu(r-1)-(\mu-\nu)(N+1)} 2^{\nu d}\left\langle 2^{\nu} \tilde{y}\right\rangle^{-M} .
\end{aligned}
$$

Thus we obtain the claim.

REMARK 2.37. The Proof of the Lemma stays unchanged for the case $r=1$, provided the estimates on $|\alpha|=1$ and $|\alpha|=0$ are added on $u_{s}$ and $c_{s}$ respectively. In this case there is no improvement of decay. But note that in our later applications this improvement is usually not going to be used.

Proposition 2.38. Let $p \in I_{1,1}^{m_{1}, \rho_{1}}\left(\mathbb{R}^{n}, \mathbb{R}^{n-1}\right)$ and $\kappa$ be a diffeomorphism of conormal type and order $\left(m_{2}, \rho_{2}\right)$ with $m_{2}<-2$ and $m_{1}-\rho_{1}<-1$, then for the respective component in (5) if $t \geq T$, the essential bound, we obtain

$$
\int_{t}^{\infty} p_{G, s}\left(\kappa_{s}\right) \mathrm{d} \ln (s) \in G^{m_{1}-\rho_{1}} .
$$

Proof. For simplicity of notation introduce $2^{\nu}=s$. To obtain a Besov norm estimate, we need to consider

$$
\left\|\psi_{\mu}(D)\left[\left(\psi_{\nu}(D) p_{G}\right) \circ \kappa_{s}\right]\right\|_{L^{p}} .
$$

From the assumptions and uniform $L^{p}$-boundedness of the composition with $\kappa_{s}$ for $s \geq T$ and the operator $\psi_{\mu}(D)$, we immediately obtain

$$
\left\|\psi_{\mu}(D)\left[\left(\psi_{\nu}(D) p_{G}\right) \circ \kappa_{s}\right]\right\|_{L^{p}} \lesssim 2^{\nu\left(m_{1}-\rho_{1}+1-1 / p\right)} .
$$

These estimates are already sufficient for $2^{\mu} \lesssim 2^{\nu}$. Let $\tilde{\psi}_{\nu}=\int_{\nu / 4}^{4 \nu} \psi_{\vartheta} \mathrm{d} \ln (\vartheta)$, satisfying $\psi_{\nu} \cdot \tilde{\psi}_{\nu}=\psi_{\nu}$. For $2^{\mu} \gg 2^{\nu}$ we apply Lemma 2.36 and obtain

$$
\begin{aligned}
\left\|\psi_{\mu}(D)\left[\left(\psi_{\nu}(D) p_{G}\right) \circ \kappa_{s}\right]\right\|_{L^{p}} & \lesssim\left\|\int K(x, x+y) \tilde{\psi}_{\nu}(D) p_{G}(y) \mathrm{d} y\right\|_{L^{p}} \\
& \lesssim\|K(x, x+y)\|_{L^{1}\left(\mathbb{R}_{y}^{n} ; L^{\infty}\left(\mathbb{R}_{x}^{n}\right)\right)}\left\|\tilde{\psi}_{\nu}(D) p_{G}\right\|_{L^{p}} \\
& \lesssim 2^{\nu\left(m_{1}-\rho_{1}+1-1 / p\right)+\mu\left(m_{2}+2\right)-(\mu-\nu) \ell} .
\end{aligned}
$$

Now we use the two types of estimates and integrate. We recall the condition $\mu \geq \nu+d_{0}$ for the second estimate and obtain:

$$
\int_{\ln (t)}^{\mu-d_{0}} 2^{\nu\left(m_{1}-\rho_{1}+1-1 / p+\ell\right)+\mu\left(m_{2}+2\right)-\mu \ell} \mathrm{d} \nu \lesssim 2^{\mu\left(m_{1}-\rho_{1}+1-1 / p+m_{2}+2\right)}
$$


and for the first estimate:

$$
\int_{\mu-d_{0}}^{\infty} 2^{\nu\left(m_{1}-\rho_{1}+1-1 / p\right)} \mathrm{d} \nu \lesssim 2^{\mu\left(m_{1}-\rho_{1}+1-1 / p\right)}
$$

which gives us the proper Besov space estimate.

This finally allows us to complete the proof of Theorem 2.29 .

END OF Proof of 2.29. We recall the four integral components from (5)

$$
p \circ \kappa=p_{t} \circ \kappa_{t}+u_{\kappa, C}+u_{p, C}+u_{\kappa, G}+u_{p, G} .
$$

We obtain with Propositions 2.33, 2.34, 2.35 and 2.38, that

$$
u_{\kappa, C} \in\left\{\begin{array} { l } 
{ I _ { 1 , 1 } ^ { m _ { 2 } , \operatorname { m i n } ( \rho _ { 2 } , - m _ { 1 } - 2 + \rho _ { 1 } ) } } \\
{ I _ { 1 , 1 } ^ { m _ { 2 } + m _ { 1 } + 2 , \operatorname { m i n } ( \rho _ { 1 } , \rho _ { 2 } ) } } \\
{ I _ { 1 , 1 } ^ { m _ { 2 } + \epsilon , \operatorname { m i n } ( \rho _ { 1 } , \rho _ { 2 } ) + \epsilon } }
\end{array} \quad u _ { \kappa , G } \in \left\{\begin{array}{ll}
G^{m_{2}-\rho_{2}} & m_{1}+2<0 \\
G^{m_{1}+m_{2}+2-\rho_{2}} & m_{1}+2>0 \\
G^{\epsilon+m_{2}-\rho_{2}} & m_{1}+2=0
\end{array}\right.\right.
$$

and

$$
u_{p, C} \in I_{1,1}^{m_{1}, \min \left(\rho_{1}, \rho_{2}-m_{2}-2\right)} \quad u_{p, G} \in G^{m_{1}-\rho_{1}} .
$$

So we obtain as common symbol regularity $m=\max \left(m_{i}\right)$ and for the $G$ regularity we obtain

$$
m-\rho= \begin{cases}\max \left(m_{1}-\rho_{1}, m_{2}-\rho_{2}\right) & m_{1}+2<0 \\ \max \left(m_{1}-\rho_{1}, m_{2}-\rho_{2}+m_{1}+2\right) & m_{1}+2>0 \\ \max \left(m_{1}-\rho_{1}, m_{2}-\rho_{2}+\epsilon\right) & m_{1}+2=0\end{cases}
$$

which is the claim.

REMARK 2.39. Note that using the decomposition in (5) as a formal computation for $p \circ \kappa$ for $p \notin C(\mathbb{R})$, the condition $m_{1}<-1$ can be relaxed to $m_{1}-\min \left(\rho_{1},-m_{2}-2\right)<$ -1 .

The provided lemmas now immediately yield the following slightly different composition theorem.

TheOREM 2.40. Let $\rho>0, m<-2$ and $f \in C_{*}^{-m+\rho}\left(\mathbb{R}^{d}\right)$ and $u=\left(u_{i}\right)_{i=1}^{d} \in$ $I_{1,1}^{m, \rho}\left(\mathbb{R}^{n}, \mathbb{R}^{n-1} ; \mathbb{R}^{d}\right)$, then

$$
f\left(u_{1}(x), \ldots, u_{d}(x)\right) \in I_{1,1}^{m, \rho}\left(\mathbb{R}^{n}, \mathbb{R}^{n-1}\right)
$$

Proof. Without loss of generality $f$ is compactly supported as $u_{i}$ have compact range. Use the parallel construction for a smooth approximation as in Theorem 2.29

$$
\begin{aligned}
u_{i, s} & =\varphi(D / s) u_{i, G}+\varphi\left(D_{x^{\prime}} / s\right) u_{i, C}, \quad u_{s}=\left(u_{i, s}\right)_{i=1}^{d}, \quad f_{s}=\varphi(D / s) f \\
f(u) & =\lim _{s \rightarrow \infty} f_{s}\left(u_{s}\right) \\
& =f_{t}\left(u_{t}\right)+\int_{t}^{\infty}(\psi(D / s) f) \circ u_{s}+\sum_{j=1}^{d} \partial_{j} f_{s}\left(u_{s}\right)\left(\psi(D / s) u_{j, G}+\psi\left(D_{x^{\prime}} / s\right) u_{j, C}\right) \mathrm{d} \ln (s) .
\end{aligned}
$$


Now applying Lemma 2.36 to the first integral component, we obtain the estimate

$$
\begin{aligned}
& \left\|\psi_{\mu}(D) \int_{t}^{\infty}(\psi(D / s) f) \circ u_{s} \mathrm{~d} \ln (s)\right\|_{L^{p}} \\
& \lesssim\left\|\psi_{\mu}(D) \int_{t}^{C 2^{\mu}}(\psi(D / s) f) \circ u_{s} \mathrm{~d} \ln (s)\right\|_{L^{p}}+\int_{C 2^{\mu}}^{\infty}\left\|K_{s}(x, \cdot)\right\|_{L^{1}\left(\mathbb{R}^{d}\right)}\|\psi(D / s) f\|_{L^{p}} \mathrm{~d} \ln (s) \\
& \lesssim \int_{t}^{C 2^{\mu}} s^{m-\rho} \mathrm{d} \ln (s)+\int_{C 2^{\mu}}^{\infty} 2^{\mu(m+2)}\left(s 2^{-\mu}\right)^{\ell} s^{m-\rho} \mathrm{d} \ln (s) \lesssim 2^{\mu(m-\rho)} \lesssim 2^{\mu(m-\rho+1-1 / p)} .
\end{aligned}
$$

Lemma 2.32 now yields symbol estimates for the symbols on the second component and parallel to the proofs of Propositions 2.33 and 2.35 we obtain the claim.

We already mentioned that we like to interpret $\kappa$ as a transition function but therefore not only stability under concatenation with other transition functions but also stability under inversion of the transition function is necessary to be shown. Therefore we provide the following a priori estimates for $\kappa_{s}^{-1}$. Note that from Remark 2.31 we already learned that $\kappa_{s}$ is invertible for $s \geq T$.

Lemma 2.41. Let $\kappa_{s}$ be given according to (1) and (3). Let $s \geq T$ where $T$ is the essential bound. Then $\iota_{s}=\kappa_{s}^{-1}$ obeys the estimates

$$
\begin{aligned}
\left|\partial_{x}^{\alpha} \iota_{s}(x)\right| & \lesssim s^{\left(m+1+\left|\alpha^{\prime}\right|+\left(\left|\alpha^{\prime \prime}\right|-\rho\right)^{+}\right)_{\epsilon}^{+}} \\
\left\|\partial_{x^{\prime}}^{\alpha^{\prime}} \iota_{s}\left(\cdot, x^{\prime}\right)\right\|_{C_{*}^{-m-1+\rho-\left|\alpha^{\prime}\right|}} & \lesssim 1 \quad \forall\left|\alpha^{\prime}\right|+m+1<0
\end{aligned}
$$

analogously to $\kappa_{s}$. Also we can give $\iota_{s}$ as

$$
\iota_{s}(x)=\left(\iota_{s}^{\prime \prime}(x), x^{\prime} G_{s}(x)\right)
$$

with the analogue inherited estimates on $G_{s}$ as those for $F_{s}$.

Proof. We will inductively show the estimates. We have

$$
\nabla \iota_{s}(x)=\left(\nabla \kappa_{s}\right)^{-1}\left(\iota_{s}(x)\right)
$$

and from this we obtain the recursive equation (again omitting combinatorial factors)

$$
\partial_{x}^{\alpha} \nabla \iota_{s}(x)=\sum\left[\partial_{x}^{\beta}\left(\nabla \kappa_{s}\right)^{-1}\right]\left(\iota_{s}(x)\right) \prod_{j=1}^{|\beta|} \partial_{x}^{\alpha_{j}}\left(\iota_{s}(x)\right) \ell_{j} .
$$

Here the sum is over all $1 \leq|\beta| \leq|\alpha|$ with $\beta=\sum_{j} e_{\ell_{j}}$, with $e_{k}$ being the $k$-th unit vector, and $\alpha=\sum_{j} \alpha_{j}$ with $\left|\alpha_{j}\right| \geq 1$.

First note that, as $\kappa$ leaves the hyperplane $x^{\prime}=0$ invariant, $\frac{\partial \kappa_{s, x^{\prime \prime}}}{\partial_{x^{\prime \prime}}}$ is invertible near $x^{\prime}=0$ with uniform bounds for $s \geq T$. Due to the inversion formula for block matrices we obtain

$$
\left[\begin{array}{cc}
\frac{\partial \kappa_{s, x^{\prime \prime}}}{\partial_{x^{\prime \prime}}} & \frac{\partial \kappa_{s, x^{\prime \prime}}}{\partial \kappa_{s}^{\prime}} \\
\frac{\partial \kappa_{s, x^{\prime}}}{\partial_{x^{\prime \prime}}} & \frac{\partial \kappa_{s, x^{\prime}}}{\partial_{x^{\prime}}}
\end{array}\right]^{-1}=\left[\begin{array}{ll}
A & B \\
C & D
\end{array}\right]^{-1}=\left[\begin{array}{cc}
A^{-1}+A^{-1} B S^{-1} C A^{-1} & -A^{-1} B S^{-1} \\
-S^{-1} C A^{-1} & S^{-1}
\end{array}\right]
$$

with $S=D-C A^{-1} B$. We have an entry in the lower left $-S^{-1} C A^{-1}$, with $C=x^{\prime} \frac{\partial F_{s}^{\prime}}{\partial x^{\prime \prime}}$ and thus is proportional to $x^{\prime}$ as is the perturbation from $A^{-1}$ of the upper 
left entry. Note that this $x^{\prime}$-like factor counters the reduction of regularity with respect to $x^{\prime}$-derivatives coming from the conormal part, i.e. in the factors $S, B$ and $D$.

Now assume the estimate is established for $|\alpha| \leq k$. Using the recursive equation (8) we conclude that each factor on the right side obeys said estimates and the vanishing condition. Now for each $x^{\prime}$-derivative acting on $\left(\nabla \kappa_{s}\right)^{-1}$ we either also take a $x^{\prime}$-derivative on the corresponding $\left(\iota_{s}\right)_{e_{x^{\prime}}}$ factor or we obtain a factor

$$
\partial_{x^{\prime \prime}}^{\alpha_{j}^{\prime \prime}} \frac{\partial \iota_{s}^{\prime}(x)}{\partial x^{\prime \prime}}=-\partial_{x^{\prime \prime}}^{\alpha_{j}^{\prime \prime}}\left(S^{-1} C A^{-1}\right)\left(\iota_{s}(x)\right)
$$

vanishing in $x^{\prime}=0$ of first order, thus the amount of $x^{\prime}$-derivatives effectively acting on $\left(\nabla \kappa_{s}\right)^{-1}$ is bounded by $\left|\alpha^{\prime}\right|$, so we may assume $\left|\beta^{\prime}\right| \leq\left|\alpha^{\prime}\right|$. With convexity of the estimate in $\left(\alpha_{j}\right)$ we can assume that $\left|\alpha_{j}\right|=1$ for all $j>1$. And as stated above we can set

$$
\begin{aligned}
& \left|\alpha_{1}^{\prime}\right|=\left|\alpha^{\prime}\right|-\left|\beta^{\prime}\right|+1_{\left\{\left|\beta^{\prime}\right|>0\right\}} \\
& \left|\alpha_{1}^{\prime \prime}\right|=\left|\alpha^{\prime \prime}\right|-\left|\beta^{\prime \prime}\right|+1_{\left\{\left|\beta^{\prime}\right|=0\right\}}
\end{aligned}
$$

We obtain as an estimate for an entry regarding an $x^{\prime \prime}$-derivative of $\nabla \iota_{s}$

$$
\begin{array}{r}
\left.\sum s^{\left(m+1+\left|\beta^{\prime}\right|+\left(\left|\beta^{\prime \prime}\right|+1-\rho\right)^{+}\right)_{\epsilon}^{+}} s^{\left(m+1+\left|\alpha^{\prime}\right|-\left|\beta^{\prime}\right|+1_{\left\{\left|\beta^{\prime}\right|>0\right\}}\right.}+\left(\left|\alpha^{\prime \prime}\right|-\left|\beta^{\prime \prime}\right|+1_{\left\{\left|\beta^{\prime}\right|=0\right\}}-\rho\right)^{+}\right)_{\epsilon}^{+} \\
\lesssim s^{\left(m+1+\left|\alpha^{\prime}\right|+\left(\left|\alpha^{\prime \prime}\right|+1-\rho\right)^{+}\right)_{\epsilon}^{+}}
\end{array}
$$

and analogously for an entry regarding an $x^{\prime}$-derivative of $\nabla \iota_{s}$

$$
\begin{array}{r}
\left.\sum s^{\left(m+2+\left|\beta^{\prime}\right|+\left(\left|\beta^{\prime \prime}\right|-\rho\right)^{+}\right)_{\epsilon}^{+}} s^{\left(m+1+\left|\alpha^{\prime}\right|-\left|\beta^{\prime}\right|+1_{\left\{\left|\beta^{\prime}\right|>0\right\}}\right.}+\left(\left|\alpha^{\prime \prime}\right|-\left|\beta^{\prime \prime}\right|+1_{\left\{\left|\beta^{\prime}\right|=0\right\}}-\rho\right)^{+}\right)_{\epsilon}^{+} \\
\lesssim s^{\left(m+2+\left|\alpha^{\prime}\right|+\left(\left|\alpha^{\prime \prime}\right|-\rho\right)^{+}\right)_{\epsilon}^{+}}
\end{array}
$$

Which provides the induction step for $x^{\prime}$ small. Now for $x^{\prime}$ away from 0 , we have $\iota_{s}^{\prime}(x)$ away from 0 . By almost locality of smoothing, we have the improved estimates

$$
\left|\partial_{y}^{\alpha} \kappa(y)_{\mid y=\iota_{s}^{\prime}(x)}\right| \lesssim s^{(m-\rho+1+|\alpha|)_{\epsilon}^{+}} .
$$

Now (8) directly implies the induction step, without the need for a distinction between the components.

The initial Hölder-Zygmund estimate for $\left|\alpha^{\prime}\right|=0$ is obtained by estimating the $C_{*}^{-m-2+\rho}$ norm of

$$
\frac{\partial \iota_{s}\left(\cdot, x^{\prime}\right)}{\partial x^{\prime \prime}}=\left[\begin{array}{c}
A^{-1}+A^{-1} B S^{-1} C A^{-1} \\
-S^{-1} C A^{-1}
\end{array}\right]\left(\iota_{s}^{\prime \prime}\left(\cdot, x^{\prime}\right), \iota_{s}^{\prime}\left(\cdot, x^{\prime}\right)\right)
$$

with the above estimates already proving that $\left\|\iota_{s}\left(x^{\prime}, \cdot\right)\right\|_{C_{*}^{-m-1+\rho-\epsilon}} \lesssim 1$, this yields the claim, as the outer function in variables $\left(x^{\prime \prime}(y), x^{\prime}(y)\right)$ with $\partial_{y} x^{\prime}(y) \approx x^{\prime}$ is bounded in $C_{*}^{-m-1+\rho}$, as shown in the proof of Lemma 2.32. To obtain the HölderZygmund estimate for some $\left|\alpha^{\prime}\right|>0$, we only need to estimate the $C_{*}^{-m-1+\rho-\left|\alpha^{\prime}\right|}$ norm of

$$
\left[\partial_{x}^{\beta}\left(\nabla \kappa_{s}\right)^{-1}\right]\left(\iota_{s}(x)\right)
$$

for $|\beta| \leq\left|\alpha^{\prime}\right|-1$ with the same arguments. The other factors again are already bounded in $C_{*}^{-m-1+\rho-\left|\alpha^{\prime}\right|}$ by the above estimates. 
The presentation of $\iota_{s}$ follows immediately from

$$
\iota_{s}^{\prime}(x)=\int_{0}^{1} \frac{\mathrm{d}}{\mathrm{d} t} \iota_{s}^{\prime}\left(x^{\prime \prime}, t x^{\prime}\right) \mathrm{d} t=x^{\prime} \int_{0}^{1}\left(\partial_{x^{\prime}} \iota_{s}^{\prime}\right)\left(x^{\prime \prime}, t x^{\prime}\right) \mathrm{d} t
$$

with the already provided estimates.

With these a priori estimates we can prove stability under inversion with the already familiar scheme.

THEOREM 2.42. Let $\kappa$ be a diffeomorphism of conormal type and order $(m, \rho)$, with $m<-2$ then $\kappa^{-1}$ is a diffeomorphism of conormal type and order $(m, \rho)$.

Proof. We have to find the components $\iota=\iota_{C}+\iota_{G}+\iota_{\infty}$ with $\iota_{C}+\iota_{G} \in I_{1,1}^{m, \rho}$ and $\iota_{\infty} \in C^{\infty}$ with $\nabla \iota_{\infty} \in C_{b}^{\infty}$.

We take the same decomposition of $\kappa$ as in Theorem 2.29 and define $F_{s}$ and $\kappa_{s}$ according to (1) and (3) and $G_{s}$ according to Lemma 2.41. Then we obtain

$$
\begin{aligned}
\kappa^{-1}(x) & =\lim _{s \rightarrow \infty} \kappa_{s}^{-1}(x) \\
& =\kappa_{t}^{-1}(x)+\int_{t}^{\infty} \frac{\mathrm{d}}{\mathrm{d} s}\left(\kappa_{s}\right)^{-1}(x) \mathrm{d} s \\
& =\kappa_{t}^{-1}(x)-\int_{t}^{\infty}\left[\left(\nabla \kappa_{s}(y)\right)^{-1} \frac{\mathrm{d}}{\mathrm{d} s} \kappa_{s}(y)\right]_{y=\kappa_{s}^{-1}(x)} \mathrm{d} s .
\end{aligned}
$$

Now the terms under the integral can be estimated separately, we obtain for $2^{\mu} \gtrsim s$

$$
\begin{aligned}
\| \psi_{\mu}(D)\left[( \nabla \kappa _ { s } ) ^ { - 1 } \left(\psi\left(D_{x^{\prime \prime}} / s\right) \kappa^{\prime \prime}\right.\right. & \left.\left.+x^{\prime} \psi(D / s) F_{G}\right)\right] \circ \kappa_{s}^{-1}(x) \|_{L^{p}} \\
& \lesssim 2^{\mu(m+2)}\left(s 2^{-\mu}\right)^{\ell} s^{m-\rho+1-1 / p}
\end{aligned}
$$

by Lemma 2.36 . And for $2^{\mu} \lesssim s$, we obtain naturally

$$
\left\|\psi_{\mu}(D)\left[\left(\nabla \kappa_{s}\right)^{-1}\left(\psi\left(D_{x^{\prime \prime}} / s\right) \kappa^{\prime \prime}+x^{\prime} \psi(D / s) F_{G}\right)\right] \circ \kappa_{s}^{-1}(x)\right\|_{L^{p}} \lesssim s^{m-\rho+1-1 / p} .
$$

So again taking the $s$ integral, we obtain

$$
\int_{t}^{\infty}\left[\left(\nabla \kappa_{s}\right)^{-1}\left(\psi\left(D_{x^{\prime \prime}} / s\right) \kappa^{\prime \prime}+x^{\prime} \psi(D / s) F_{G}\right)\right] \circ \kappa_{s}^{-1}(x) \mathrm{d} \ln (s) \in G^{m-\rho} .
$$

And it remains to estimate the symbolic part

$$
\begin{aligned}
& \int_{t}^{\infty}\left[\left(\nabla \kappa_{s}\right)^{-1}\left(x^{\prime} \psi\left(D_{x^{\prime}} / s\right) F_{C}\right)\right] \circ \kappa_{s}^{-1}(x) \mathrm{d} \ln (s) \\
= & \int_{t}^{\infty}\left(\nabla \kappa_{s}\right)^{-1}\left(\kappa_{s}^{-1}(x)\right) \int e^{i x^{\prime} G_{s}(x) \xi^{\prime}} D_{\xi^{\prime}}\left[\psi\left(\xi^{\prime} / s\right) a\left(\kappa_{s}^{-1}(x)^{\prime \prime}, \xi^{\prime}\right)\right] \mathrm{d} \xi^{\prime} \mathrm{d} \ln (s) \\
= & e^{i x^{\prime} \eta^{\prime}} \int_{t}^{\infty}\left(\nabla \kappa_{s}\right)^{-1}\left(\kappa_{s}^{-1}(x)\right) D_{\eta^{\prime}}\left[\psi\left(G_{s}(x) \eta^{\prime} / s\right) a\left(\kappa_{s}^{-1}(x)^{\prime \prime}, G_{s}(x) \eta^{\prime}\right)\right] \mathrm{d} \ln (s) \mathrm{d} \eta^{\prime}
\end{aligned}
$$

which can be estimated just as in Proposition 2.34. Now $\iota_{\infty}(x)=\kappa_{t}^{-1}(x)$ immediately satisfies $\nabla \iota_{\infty} \in C_{b}^{\infty}$ with 2.41 as $t$ is fixed, which provides the claim. 
4.1. Conormal distributions on curved hypersurfaces. An immediate consequence of Theorem 2.29 and 2.42 is that diffeomorphisms of conormal type form a group. We are thus able to give a definition of a geometry endowed with a structure of conormal distributions of type $(1,1)$.

DEFINITION 2.43. Let $(\Omega, \Sigma)$ be a manifold with submanifold $\Sigma$ of codimension 1 and atlas $\mathcal{A}$ such that the transition functions $\kappa_{1}^{-1} \kappa_{2}$ are diffeomorphism of conormal type and order $(m, \rho)$. Then define

$$
u \in{ }_{\operatorname{loc}} I_{1,1}^{m, \rho}(\Omega, \Sigma) \Leftrightarrow u \circ \kappa \in{ }_{\operatorname{loc}} I_{1,1}^{m, \rho}\left(\mathbb{R}^{n}, \mathbb{R}^{n-1}\right) \forall \kappa \in \mathcal{A} .
$$

REMARK 2.44. To avoid issues with smoothness conditions approaching order $-m+\rho$ like in $B_{p, \infty}^{-m+\rho+1-1 / p}$, one should attribute the structure to a $C_{*}^{-m+\rho}$-atlas and consider the transformation under general diffeomorphisms of conormal type only as an additional computation tool. Especially as the underlying manifold $\Omega$ itself will usually obey a smooth structure and the $C_{*}^{-m+\rho}$ atlases track this structure rather faithfully, in the sense that all Besov spaces up to order $-m+\rho$ are preserved.

Then the transformation behavior of our symbol is given by

$$
c\left(x, \zeta^{\prime}\right)=\int_{t}^{\infty} a\left(\kappa_{s},\left(F_{s}^{\prime}\right)^{-1} \zeta^{\prime}\right)\left(F_{s}^{\prime}\right)^{-1} \psi\left(\left(F_{s}^{\prime}\right)^{-1} \zeta^{\prime} / s\right) \mathrm{d} \ln (s)
$$

whilst all other components of (5) yield only remainder terms. Thus the geometry on which a (principal) symbol is to be defined is not the conormal bundle but a (class of) smooth approximation(s) of this bundle. In local coordinates we are still able to do the usual computations like in (1) and they make perfect sense.

REMARK 2.45. Given a uniform structure, which is given by a partition of unity $\varpi_{j} \in C_{*}^{-m+\rho}(\Omega)$ with compact support and $\sum_{j} \varpi_{j}=1$, we can define the uniform $I_{1,1}^{m, \rho}(\Omega, \Sigma)$ space accordingly.

We can also construct embeddings for non smooth hypersurfaces $\Sigma \subset \mathbb{R}^{n}$, which allow the study of our distributions in a smooth context.

EXAMPLE 2.46. Let $f\left(x^{\prime \prime}\right) \in C_{*}^{m-\rho-1}$, then the graph $\Sigma=\left\{\left(x^{\prime \prime}, f\left(x^{\prime \prime}\right)\right) \mid x^{\prime \prime} \in \mathbb{R}^{n-1}\right\}$ of $f$ is an embedded submanifold of $\mathbb{R}^{n}$ with embedding $\kappa_{f}:\left(\mathbb{R}^{n}, \mathbb{R}^{n-1}\right) \rightarrow\left(\mathbb{R}^{n}, \Sigma\right)$ for some $\alpha \gg 0$ depending on $\|\nabla f\|_{L^{\infty}}$

$$
\kappa_{f}(x)=\left(x^{\prime \prime}, \mathfrak{E}[f](x)+\alpha x^{\prime}\right)
$$

which is smooth away from $\mathbb{R}^{n-1}$ and $\infty$-localized in the sense that $G^{m-\rho, \ell}\left(\mathbb{R}^{n}, \Sigma\right)$ can be intrinsically defined as

$$
u \in G^{m-\rho}\left(\mathbb{R}^{n}, \mathbb{R}^{n-1}\right) ;\left(\kappa^{\prime}(x)\right)^{\ell} u \in G^{m-\rho-\ell}\left(\mathbb{R}^{n}, \mathbb{R}^{n-1}\right)
$$

We conjecture that the definition of $G^{m-\rho, \ell}\left(\mathbb{R}^{n}, \Sigma\right)$ coincides with the class defined via $u \circ \kappa \in G^{m-\rho, \ell}\left(\mathbb{R}^{n}, \mathbb{R}^{n-1}\right)$ and that the here defined structure for conormal distributions of conormal type on $\Sigma$ is invariant under the choice of initial smooth coordinates. 



\section{CHAPTER 3}

\section{The Linear Cauchy Problem}

In this chapter we aim to provide techniques to analyze equations of the kind

$$
\begin{cases}\sum_{|\alpha| \leq m} a_{\alpha}(x)\left(i \partial_{x}\right)^{\alpha} u=f & \text { on }[0, T] \times \Omega \subseteq \mathbb{R}^{1+n} \\ \partial_{t}^{k} u_{t=0}=u_{k} & \text { on } \Omega \subseteq \mathbb{R}^{1+n} \text { for } 0 \leq k \leq m-1\end{cases}
$$

with data, inhomogeneity and coefficients given by conormal distributions. We are interested in such equations where the principal symbol $P(x, \xi)=\sum_{|\alpha|=m} a_{\alpha}(x) \xi^{\alpha}$ is real valued and with $x=(t, y)$ and $\xi=(\tau, \zeta)$ has $m$ different real roots $\left\{\tau_{i}(x, \zeta)\right\}_{i=1}^{m}$, i.e. the equation is strictly hyperbolic. Furthermore the coefficients $a_{\alpha}$ should be in $C^{1}$.

This ensures that given a smooth submanifold $\Sigma_{0} \subseteq\left\{\left(0, x^{\prime}\right) \in \Omega\right\}$ of dimension $n-1$ there are $m$ unique $C^{2}$ submanifolds $\Sigma_{i}$ of codimension 1 constructed as the foliation of the rays of the Hamiltonian vector field with initial conditions given by the following sections in the cosphere bundle $\Gamma_{i}: \Sigma_{0} \rightarrow S^{*} \Sigma_{0}$

$$
\Gamma_{i}\left(0, x^{\prime}\right)=\left(0, x^{\prime}, \zeta_{i}\left(x, \xi^{\prime}\right), \xi^{\prime}\right) .
$$

These submanifolds should further only intersect in $\Sigma_{0}=\Sigma_{i} \cap \Sigma_{j} \forall i \neq j$.

We will not provide solutions to this very general setup but restrict to first order equations. Some of the techniques we apply are still going to be applicable for higher order equations.

We will at first give some more technical tools, to solve such linear equations on a symbol basis and then give our toy equation, which we are then going to solve.

\section{Transport equation}

Our first step in solving a linear Cauchy problem will be to obtain approximate solutions of improving quality. This is done using approximations of the equations within the symbol calculus. We then have to solve the corresponding transport equations for the symbols.

$$
\left\{\begin{array}{l}
\partial_{t} a+r \partial_{\xi^{\prime}} a+s a=g \\
a_{\left.\right|_{t=0}}=f
\end{array}\right.
$$

Here $r \in S_{1,1}^{1, \rho}, s \in S_{1,1}^{0, \rho}$ and the datum and inhomogeneity $f, g \in S_{1,1}^{m, \rho}$. In preparing to solve (1), we give a technical Proposition.

Proposition 3.1. Let $r \in S_{1,1}^{m, \rho}\left(\mathbb{R}^{n} \times \mathbb{R}\right)$ and $\gamma^{\prime} \in C_{*}^{\rho}\left(\mathbb{R}^{n}\right)$ with $\left\langle\xi^{\prime}\right\rangle \approx\left|\gamma^{\prime}\right|$. Then

$$
\left\|r\left(x, \gamma^{\prime}(x)\right)\right\|_{C_{*}^{\rho}} \lesssim C(r, \rho)\left\langle\xi^{\prime}\right\rangle^{m-1}\left\|\gamma^{\prime}\right\|_{C_{*}^{\rho}} .
$$

Proof. Let $r_{\nu}\left(x, \xi^{\prime}\right)=\psi_{\nu}\left(D_{x}\right) r(x, \xi)$, then $r_{\nu}(x, \cdot) \in C^{\infty}(\mathbb{R})$, so we apply Proposition A.10, which is $[\mathbf{H o ̈ 0 3 b}$, Prop. 8.6.12.] for some $N>\rho$ and as 
$\left|\gamma^{\prime}(y)\right| \approx\left\langle\xi^{\prime}\right\rangle$, we have without loss of generality $r_{\nu}(x, 0)=0$ and can take the supremum restricted to this annulus, and obtain

$$
\left\|r_{\nu}\left(x, \gamma^{\prime}(\cdot)\right)\right\|_{C_{*}^{\rho}} \lesssim \sum_{j=1}^{N+1} \sup \left(\frac{\left|\psi_{\nu}(D) \partial_{\eta^{\prime}}^{j} r\left(x, \eta^{\prime}\right)\right|}{\left\langle\eta^{\prime}\right\rangle^{m-j}}\right)\left\langle\xi^{\prime}\right\rangle^{m-1}\left\|\gamma^{\prime}\right\|_{C_{*}^{\rho}},
$$

Thus we have for $\|r\|_{N, \rho}=\sup _{\nu, x, \eta^{\prime}, 1 \leq j \leq N+1} 2^{\nu \rho}\left\langle\eta^{\prime}\right\rangle^{-m+j}\left|\psi_{\nu}(D) \partial_{\eta}^{\prime j} r\left(x, \eta^{\prime}\right)\right|$,

$$
\left|\psi_{\nu}\left(D_{x}\right) \psi_{\mu}\left(D_{y}\right) r\left(x, \gamma^{\prime}(y)\right)\right| \lesssim 2^{-(\nu+\mu) \rho}\|r\|_{N, \rho}\left\langle\xi^{\prime}\right\rangle^{m-1}\left\|\gamma^{\prime}\right\|_{C_{*}^{\rho}} .
$$

Taking the trace $x=y$ is a convolution in Fourier space. Thus

$$
\begin{array}{r}
\psi_{\vartheta}(D) r\left(x, \gamma^{\prime}(x)\right)=\sum_{\max (\nu, \mu) \geq \vartheta-2} \psi_{\nu}\left(D_{x}\right) \psi_{\mu}\left(D_{y}\right) r\left(x, \gamma^{\prime}(y)\right)_{\left.\right|_{x=y}} \\
\left|\psi_{\vartheta}(D) r\left(x, \gamma^{\prime}(x)\right)\right| \lesssim 2^{-\vartheta \rho}\|r\|_{N, \rho}\left\langle\xi^{\prime}\right\rangle^{m-1}\left\|\gamma^{\prime}\right\|_{C_{*}^{\rho}}
\end{array}
$$

which yields the claim.

Proposition 3.2. The transport equation (1) has a unique solution $a \in S_{1,1}^{m, \rho}$ with $\partial_{t} a \in S_{1,1}^{m, \rho}$.

BEgin OF PROOF. We will use the method of characteristics to prove the proposition. We construct the flow $\gamma$ of the corresponding vector field and give symbol estimates on it. So $\gamma\left(t, x^{\prime \prime}, \xi ; \tau\right)=\left(\tau, x^{\prime \prime}, \gamma^{\prime}\left(t, x^{\prime \prime}, \xi ; \tau\right)\right)$, with $\gamma^{\prime}$ the solution of

$$
\begin{cases}\frac{\mathrm{d}}{\mathrm{d} \tau} \gamma^{\prime}\left(t, x^{\prime \prime}, \xi^{\prime} ; \tau\right) & =r\left(\tau, x^{\prime \prime}, \gamma^{\prime}\left(t, x^{\prime \prime}, \xi^{\prime} ; \tau\right)\right) \\ \gamma^{\prime}\left(t, x^{\prime \prime}, \xi^{\prime} ; t\right) & =\xi^{\prime}\end{cases}
$$

Then the solution $a$ is obtained via the equation

$$
\begin{cases}\frac{\mathrm{d}}{\mathrm{d} \tau} a(\gamma(\tau))+s a(\gamma(\tau)) & =g(\gamma(\tau)) \\ a(\gamma(0)) & =f(\gamma(0)) .\end{cases}
$$

Then using the symbol estimate of $r$, we obtain

$$
\frac{\mathrm{d} \gamma^{\prime}(\tau)}{\mathrm{d} \tau} \leq C\left\langle\gamma^{\prime}(\tau)\right\rangle \Rightarrow \exp (-C|\tau-t|)\left\langle\xi^{\prime}\right\rangle \lesssim\left\langle\gamma^{\prime}(\tau)\right\rangle \lesssim \exp (C|\tau-t|)\left\langle\xi^{\prime}\right\rangle
$$

And as $t, \tau$ are in a compact set, we have $\left\langle\gamma^{\prime}(\tau)\right\rangle \approx\left\langle\xi^{\prime}\right\rangle$. Now interpreting $\gamma$ as a family of symbols with parameters $t, \tau$ and symbol variables $x^{\prime \prime}, \xi^{\prime}$, we obtain

$$
\begin{cases}\left|\partial_{x^{\prime \prime}}^{\alpha^{\prime \prime}} \partial_{\xi^{\prime}}^{\beta} \frac{\mathrm{d} \gamma^{\prime}}{\mathrm{d} \tau}\right| & \lesssim \sum\left|\partial_{\xi^{\prime}}^{j} \partial_{x^{\prime \prime}}^{\alpha^{\prime \prime}} r\left(\tau, x^{\prime \prime}, \gamma^{\prime}\right)\right| \prod_{i=1}^{j}\left|\partial_{x^{\prime \prime}}^{\alpha_{i}^{\prime \prime}} \partial_{\xi^{\prime}}^{\beta_{i}} \gamma^{\prime}\right| \\ \partial_{x^{\prime \prime}}^{\alpha^{\prime \prime}} \partial_{\xi^{\prime}}^{\beta} \gamma^{\prime} & \left.\right|_{\left.\right|_{\tau=t}}=\left|\partial_{x^{\prime \prime}}^{\alpha^{\prime \prime}} \partial_{\xi^{\prime}}^{\beta} \xi^{\prime}\right|\end{cases}
$$

with the sum over all $j \in \mathbb{N}_{0}, \sum \alpha_{i}^{\prime \prime}=\alpha^{\prime \prime}$ and $\sum_{i} \beta_{i}=\beta$ with $\left|\alpha_{i}^{\prime \prime}\right|+\left|\beta_{i}\right| \neq 0$ for all $i>0$. Thus we inductively show that uniformly in $t, \tau$

$$
\left|\partial_{x^{\prime \prime}}^{\alpha^{\prime \prime}} \partial_{\xi^{\prime}}^{\beta} \gamma^{\prime}\right| \lesssim\left\langle\xi^{\prime}\right\rangle^{1-|\beta|+\left(\left|\alpha^{\prime \prime}\right|-\rho\right)^{+}}
$$

for $\left|\alpha^{\prime \prime}\right| \neq \rho$, which does not stop the induction if occurring, since we can worsen the estimate by $\left\langle\xi^{\prime}\right\rangle^{\epsilon}$ in this case with no effect on the higher order estimates as the inhomogeneity created by such a term is not dominating. And analogously for the Hölder-Zygmund estimates, we obtain

$$
\begin{cases}\left\|\partial_{\xi^{\prime}}^{\beta} \frac{\mathrm{d} \gamma^{\prime}}{\mathrm{d} \tau}\right\|_{C_{*}^{\rho}} & \lesssim \sum\left\|\partial_{\xi^{\prime}}^{j} r\left(\tau, x^{\prime \prime}, \gamma^{\prime}\right) \prod_{i=1}^{j} \partial_{\xi^{\prime}}^{\beta_{i}} \gamma^{\prime}\right\|_{C_{*}^{\rho}} \\ \left\|\partial_{\xi^{\prime}}^{\beta} \gamma_{\left.\right|_{\tau=t} ^{\prime}}^{\prime}\right\|_{C_{*}^{\rho}}^{\rho} & =\left\|\partial_{x^{\prime \prime}}^{\alpha^{\prime \prime}} \partial_{\xi^{\prime}}^{\beta} \xi^{\prime}\right\|_{C_{*}^{\rho}} .\end{cases}
$$


And thus we also inductively show using Proposition 3.1 and paraproduct estimates that uniformly in $t, \tau$

$$
\left\|\partial_{\xi^{\prime}}^{\beta} \gamma^{\prime}\right\|_{C_{*}^{\rho}} \lesssim\left\langle\xi^{\prime}\right\rangle^{1-|\beta|}
$$

Now having established that $\gamma^{\prime}(t, \cdot, \cdot ; \tau) \in S_{1,1}^{1, \rho}$ for all $t, \tau$ with uniform estimates, we obtain using the same methods on $(2)$, that $a(\gamma(t, \cdot, \cdot ; \tau)) \in S_{1,1}^{m, \rho}$ with uniform estimates in $t, \tau$ and thus as $\gamma\left(t, x^{\prime \prime}, \xi^{\prime} ; t\right)=\left(t, x^{\prime \prime}, \xi^{\prime}\right)$, we obtain for $\left|\alpha^{\prime \prime}\right| \neq \rho$

$$
\begin{aligned}
\left|\partial_{x^{\prime \prime}}^{\alpha^{\prime \prime}} \partial_{\xi^{\prime}}^{\beta} a\left(t, x^{\prime \prime}, \xi^{\prime}\right)\right| & \lesssim\left\langle\xi^{\prime}\right\rangle^{m+\left(\left|\alpha^{\prime \prime}\right|-\rho\right)^{+}-|\beta|} \\
\left|\psi_{\nu}\left(D_{x^{\prime \prime}}\right) \partial_{\xi^{\prime}}^{\beta} a\left(t, x^{\prime \prime}, \xi^{\prime}\right)\right| & \lesssim 2^{-\nu \rho}\left\langle\xi^{\prime}\right\rangle^{m-|\beta|} .
\end{aligned}
$$

Now using the equation $\partial_{t} a=g-r \partial_{\xi^{\prime}} a+s a$ iteratively, we obtain that $\partial_{t}^{\alpha_{0}} a_{\left.\right|_{t}} \in$ $S_{1,1}^{m, \rho-\left(\left|\alpha_{0}\right|-1\right)^{+}}\left(\mathbb{R}^{n-k} \times \mathbb{R}^{k}\right)$ and thus $a \in S_{1,1}^{m, \rho}\left(\left([0, T] \times \mathbb{R}^{n-k}\right) \times \mathbb{R}^{k}\right)$. Using the equation again, we also obtain $\partial_{t} a \in S_{1,1}^{m, \rho}\left(\left([0, T] \times \mathbb{R}^{n-k}\right) \times \mathbb{R}^{k}\right)$, which is the claim.

\section{First order equation}

To simplify computations we restrict to a first order equation with straight characteristic

$$
\left\{\begin{array}{l}
\partial_{t} u+p \partial_{x} u+q u=g \\
u_{\left.\right|_{t=0}}=v
\end{array}\right.
$$

With $v \in I_{1,1}^{m, \rho}\left(\mathbb{R}^{n}, \mathbb{R}^{n-1}\right),(p, q, g) \in I_{1,1}^{m, \rho}\left([0, T] \times \mathbb{R}^{n},[0, T] \times \mathbb{R}^{n-1} ; \mathbb{R}^{n} \times \mathbb{R} \times \mathbb{R}\right)$ and $p\left(t, x^{\prime \prime}, 0\right)=0$. Further $p, q, g$ shall satisfy the trace conditions $\partial_{t}^{j}(p, q, g) \in$ $C\left([0, T] ; I_{1,1}^{m, \rho-j}\left(\mathbb{R}^{n}, \mathbb{R}^{n-1} ; \mathbb{R}^{n} \times \mathbb{R} \times \mathbb{R}\right)\right)$ for all $j+m-1-\rho<0$.

To solve this equation we will use an inductive reduction, solving either a trace of $u$ on $x^{\prime}=0$ or solving a singular part using the transport equation.

TheOREM 3.3. Let $m<-2$ with $m, \rho, m-\rho \notin \mathbb{Z}$. Then there is an approximate symbolic solution $u \in I_{1,1 ; \infty}^{m, \rho}\left([0, T] \times \mathbb{R}^{n},[0, T] \times \mathbb{R}^{n-1}\right)$ to (1) which satisfies

$$
\left\{\begin{array}{l}
\partial_{t} u+p \partial_{x} u+q u=g-\tilde{g} \\
u_{\left.\right|_{t=0}}=v-\tilde{v}
\end{array}\right.
$$

with approximation errors $\tilde{g} \in G_{0}^{m-\rho}\left([0, T] \times \mathbb{R}^{n},[0, T] \times \mathbb{R}^{n-1}\right)$, satisfying the trace condition $\partial_{t}^{j} g \in C\left([0, T] ; G_{0}^{m-\rho+j}\right)\left(\mathbb{R}^{n-1}, \mathbb{R}^{n}\right)$ and $\tilde{v} \in G_{0}^{m-\rho}\left(\mathbb{R}^{n}, \mathbb{R}^{n-1}\right)$.

Proof. At first one should note that - as we are solving the equation up to $G_{0}^{m-\rho}$ and $u$ is purely symbolic - we can restrict all $v, g, q$ and $q$ to be purely symbolic, as their $G_{0}^{m-\rho}$ part will yield a remainder. Further we assume $p=x^{\prime} p_{1}$ from the Taylor expansion, with $p_{1}$ also purely symbolic.

Let $\ell \geq 0$ such that $-m-2 \leq \ell<-m-1$, define the approximation improvement parameter $s=\min (1,-m-2)$. Let $N=\ell+\lceil\rho / s\rceil$, then introduce the regularity 
coefficients $m_{j}, \rho_{j}$ and $d_{j}$

$$
\begin{aligned}
& d_{j}=\left\{\begin{array}{ll}
j & j \leq \ell \\
-\lceil m+2-s(j-\ell)\rceil & \ell<j<N \\
-\lceil m-\rho+2\rceil & j \geq N
\end{array} \quad \rho_{j}= \begin{cases}\rho & j \leq \ell \\
\rho-s(\ell-j) & \ell<j<N \\
0 & j \geq N\end{cases} \right. \\
& m_{j}-d_{j}-\rho_{j}=m-\rho
\end{aligned}
$$

We will assume that $m_{j} \notin \mathbb{Z}$ for all $j$, else we might artificially reduce $s$ such that this holds. This does not affect the qualitative statement we make, but only the quantitative approximation quality of our iteratively constructed solutions. We will give an inductive construction of $u$ in the form

$$
u=\sum_{j=0}^{N}\left(x^{\prime}\right)^{d_{j}} u_{j}
$$

where each $u_{j} \in I_{1,1 ; \infty}^{m_{j}, \rho_{j}}$ is itself an approximate solution to an equation of the type

$$
\begin{cases}\partial_{t} u_{j}+x^{\prime} p_{1} \partial_{x} u_{j}+\left(q_{C}+j p_{1}^{\prime}\right) u_{j} & =g_{j}-h_{j} \\ \left.u_{j}\right|_{t=0} & =v_{j}-w_{j}\end{cases}
$$

with the regularity of the datum and inhomogeneity given by $g_{j}, v_{j} \in I_{1,1 ; \infty}^{m_{j}, \rho_{j}}$ and $h_{j}, w_{j} \in I_{1,1 ; \infty}^{m_{j+1}+d_{j}-d_{j+1}, \rho_{j+1}}$. The error terms will satisfy $h_{j}\left(t, x^{\prime \prime}, 0\right)=w_{j}\left(x^{\prime \prime}, 0\right)=0$ with initial values $g_{0}=g$ and $v_{0}=v$.

At first we are going to find the necessary trace $\mathfrak{u}_{j}$ of $u_{j}$ in order to satisfy the condition $h_{j}\left(t, x^{\prime \prime}, 0\right)=w_{j}\left(x^{\prime \prime}, 0\right)=0$. So we restrict the equation to $x^{\prime}=0$ and obtain

$$
\begin{cases}\partial_{t} \mathfrak{u}_{j}\left(t, x^{\prime \prime}\right)+\left(q_{C}+j p_{1}^{\prime}\right)\left(t, x^{\prime \prime}, 0\right) \mathfrak{u}_{j}\left(t, x^{\prime \prime}\right) & =g_{j}\left(t, x^{\prime \prime}, 0\right) \\ \mathfrak{u}_{j}\left(t, x^{\prime \prime}\right) & =v_{j}\left(t, x^{\prime \prime}, 0\right)\end{cases}
$$

which is solvable via variation of constants with the solution $\mathfrak{u}_{j} \in C_{*}^{-m-1+\rho-d_{j}}$ in accordance with the coefficients and datum. Note that the lower regularity of $p_{1}{ }^{\prime}\left(t, x^{\prime \prime}, 0\right)$ comes only into play for the coefficients, when $j \neq 0$. Thus the first approximation is $u_{j}^{1}=\mathfrak{E}\left(\mathfrak{u}_{j}\right) \in I_{1,1 ; \infty}^{m-\rho+d_{j}}$. Letting the opertor act on $u_{j}^{1}$, we obtain for $j \neq 0$

$$
g_{j}^{1}=\partial_{t} u_{j}^{1}+x^{\prime} p_{1} \partial_{x} u_{j}^{1}+\left(q_{C}+j p_{1}^{\prime}\right) u_{j}^{1} \in I_{1,1 ; \infty}^{m+1, \rho-d_{j}+1} \subseteq I_{1,1 ; \infty}^{m_{j}, \rho_{j}}
$$

For $j=0$ we obtain $g_{0}^{1} \in I_{1,1 ; \infty}^{m, \rho}$. Now we already have $g_{j}, g_{j}^{1} \in I_{1,1 ; \infty}^{m_{j+1}+d_{j}-d_{j+1}, \rho_{j+1}}$ if $j<\ell$ and can set $u_{j}=u_{j}^{1}, h_{j}=g_{j}-g_{j}^{1}$ and $w_{j}=v_{j}-u_{\left.j\right|_{t=0} ^{1}}$.

If $j \geq \ell$, we need to eliminate the principal symbol of $g_{j}$ and improve the order of the remainder by $s$. We make the ansatz $u_{j}=u_{j}^{1}+u_{j}^{2}$, with $a\left(t, x^{\prime \prime}, \xi^{\prime}\right) \in S_{1,1}^{m_{j}, \rho_{j}}$ the total symbol of $u_{j}^{2}$ with $u_{j}^{2}\left(t, x^{\prime \prime}, 0\right)=0$ and $\partial_{t} a \in S_{1,1}^{m_{j}, \rho_{j}}$. Then with the help of paraproducts we analyze the symbol part of

$$
\begin{aligned}
g_{j}^{2} & =\partial_{t} u_{j}^{2}+p_{1} x^{\prime} \partial_{x} u_{j}^{2}+\left(q_{C}+j p_{1}^{\prime}\right) u_{j}^{2} \in I_{1,1}^{m_{j}, \rho_{j}} \\
\sigma\left(g_{j}^{2}\right) & \equiv \partial_{t} a+\left(p_{1}^{\prime}\right)_{\chi}\left(x^{\prime \prime}, 0, \xi^{\prime}\right)\left(D_{\xi^{\prime}}\left(i \xi^{\prime}\right) a\right)+\left(q_{C}+j p_{1}^{\prime}\right)_{\chi}\left(x^{\prime \prime}, 0, \xi^{\prime}\right) a \quad \bmod S_{1,1}^{m_{j}-s, \rho_{j}-s}
\end{aligned}
$$


Now take $b\left(t, x^{\prime \prime}, \xi^{\prime}\right) \in S_{1,1}^{m_{j}, \rho_{j}}$ a full symbol of $g_{j}-g_{j}^{1}$ and $c\left(x^{\prime \prime}, \xi^{\prime}\right) \in S_{1,1}^{m_{j}, \rho_{j}}$ a full symbol of $v_{j}$. Then we obtain the equation

$$
\begin{cases}\partial_{t} a_{0}+\left(p_{1}^{\prime}\right)_{\chi}\left(x^{\prime \prime}, 0, \xi^{\prime}\right)\left(D_{\xi^{\prime}}\left(i \xi^{\prime}\right) a_{0}\right)+\left(q_{C}+j p_{1}^{\prime}\right)_{\chi}\left(x^{\prime \prime}, 0, \xi^{\prime}\right) a_{0} & =b \\ a_{\left.0\right|_{t=0}} & =c\end{cases}
$$

for $a_{0}$. Which by Proposition 3.2 has a solution $a_{0} \in S_{1,1}^{m_{j}, \rho_{j}}$, adjusting the a priori solution $a_{0}$ with trace $\mathfrak{a}\left(x^{\prime \prime}\right)=\int a_{0}\left(x^{\prime \prime}, \xi^{\prime}\right) \mathrm{d} \xi^{\prime}$ by $a=a_{0}-\mathfrak{e}(\mathfrak{a})$, we obtain an approximate solution $u_{j}=u_{j}^{1}+u_{j}^{2}$ with $h_{j}=g_{j}-g_{j}^{1}-g_{j}^{2}$. And we have $w_{j}=v_{j}-u_{\left.j\right|_{t=0}} \in I_{1,1 ; \infty}^{m-\rho+d_{j}}$.

Now if $m_{j}-s+\left(s_{j}-s\right)^{-}>-2$, we define $g_{j+1}=h_{j}$ and $v_{j+1}=w_{j}$, if however $m_{j}-s+\left(s_{j}-s\right)^{-}<-2$, we can use the Taylor formula to obtain $h_{j}=\left(x^{\prime}\right) g_{j+1}+h_{j, G}$ and $w_{j}=\left(x^{\prime}\right) v_{j+1}+w_{j, G}$, where $w_{j, G}, h_{j, G} \in G_{0}^{m_{j}-\rho_{j}, \infty}$. Which finishes the inductive construction.

Now we have

$$
\begin{aligned}
\partial_{t}\left(x^{\prime}\right)^{d_{j}} u_{j}+x^{\prime} p_{1} \partial_{x}\left(x^{\prime}\right)^{d_{j}} u_{j}+q_{C}\left(x^{\prime}\right)^{d_{j}} u_{j} & =\left(x^{\prime}\right)^{d_{j}}\left[\partial_{t} u_{j}+x^{\prime} p_{1} \partial_{x} u_{j}+\left(q_{C}+d_{j} p_{1}^{\prime}\right) u_{j}\right] \\
& =\left(x^{\prime}\right)^{d_{j}} g_{j}-\left(x^{\prime}\right)^{d_{j+1}} g_{j+1}-\left(x^{\prime}\right)^{d_{j}} h_{j, G}
\end{aligned}
$$

and obtain

$$
\begin{cases}\partial_{t} u+x^{\prime} p_{1} \partial_{x} u+q_{C} u & =g-\left(x^{\prime}\right)^{d_{N}} h_{N}-\sum_{j=0}^{N-1}\left(x^{\prime}\right)^{d_{j}} h_{j, G} \\ u_{\left.\right|_{t=0}} & =v-\left(x^{\prime}\right)^{d_{N}} w_{N}-\sum_{j=0}^{N-1}\left(x^{\prime}\right)^{d_{j}} w_{j, G}\end{cases}
$$

As we already have $p_{G} \partial_{x} u+q_{G} u \in G_{0}^{m-\rho}$, we obtain the claim.

\section{The reduced problem}

Thus it remains to find a solution to (1), with $\tilde{v}, \tilde{g} \in G_{0}^{m-\rho}$. For this case, we need some technical preparations.

Definition 3.4. Let $1<p \leq \infty, m \in \mathbb{R}$ and $\rho \geq 0$, define for $m_{\alpha}=m-\left|\alpha^{\prime}\right|+$ $(|\alpha|-\rho)^{+}$the following seminorms

$$
\|u\|_{\alpha, p}=\left\|\left\langle x^{\prime}\right\rangle^{-\left|\alpha^{\prime}\right|}\left(x^{\prime}\right)^{\alpha^{\prime}} \partial_{x^{\prime \prime}}^{\alpha^{\prime \prime}} u\right\|_{\text {unif } B_{p, \infty}^{-m_{\alpha}-1+1 / p}}+\left\|\left\langle x^{\prime}\right\rangle^{-\left|\alpha^{\prime}\right|}\left(x^{\prime}\right)^{\alpha^{\prime}} \partial_{x^{\prime \prime}}^{\alpha^{\prime \prime}} u\right\|_{C_{*}^{-m_{\alpha}-1}}
$$

and define the space

$$
K_{p}^{m, \rho}=\left\{u \in \mathcal{S}^{\prime}\left(\mathbb{R}^{n}\right) ;\|u\|_{K_{p}^{m, \rho}}=\sum_{|\alpha| \leq\lceil\rho\rceil}\|u\|_{\alpha, p}<\infty\right\} .
$$

A diffeomorphism $\kappa$ is called a diffeomorphism of $K_{p}^{m, \rho}$-type, if

$$
\kappa(x)=\left(x^{\prime \prime}+\kappa^{\prime \prime}(x), x^{\prime} F(x)\right)
$$

with $\kappa^{\prime \prime}(x) \in K_{p}^{m, \rho}$ and $\left\langle x^{\prime}\right\rangle F(x) \in K_{p}^{m+1, \rho+1}, F>0$. With abuse of notation define $\|\kappa\|_{K_{p}^{m, \rho}}=\left\|\kappa^{\prime \prime}\right\|_{K_{p}^{m, \rho}}+\left\|\left\langle x^{\prime}\right\rangle F\right\|_{K_{p}^{m+1, \rho}}$.

For these distributions the theorems for nonlinear superposition and multiplications still hold correspondingly. 
Proposition 3.5. Let $u_{1} \in K_{p}^{m_{1}, \rho_{1}}$ and $u_{2} \in K^{m_{2}, \rho_{2}}$ with $m_{i}<-1$, then

$$
u_{1} u_{2} \in K_{p}^{m, \rho}
$$

with $m=\max \left(m_{1}, m_{2}\right)$ and $m-\rho=\max \left(m_{1}-\rho_{1}, m_{2}-\rho_{2}\right)$.

If $m_{1}<m_{2}, \rho_{1}=0$ and $u_{1}$ vanishes of order $N<-m_{1}-1$ in $x^{\prime}=0$, then

$$
u_{1} u_{2} \in K_{p}^{\max \left(m_{1}, m_{2}-N+\left(\rho_{2}-N\right)^{-}\right)} .
$$

Proof. We use paramultiplication and obtain

$$
u_{1} u_{2}=u_{1, \chi}(x, D) u_{2}+u_{2, \chi}(x, D) u_{1}+u_{2, \Phi}(x, D) u_{1} .
$$

Now from the properties of $u_{1}$ we obtain for $q_{i}(x, \xi)=u_{i, \chi}(x, \xi)$

$$
\partial_{x^{\prime \prime}}^{\alpha^{\prime \prime}} q_{i}(x, \xi) \in S_{1,1}^{\left(m_{i}+1+\left(\left|\alpha^{\prime \prime}\right|-\rho_{i}\right)^{+}\right)_{\epsilon}^{+}} \text {. }
$$

So using the commutation relations $\left[x^{\prime}, q(x, D)\right]=i\left(\partial_{\xi^{\prime}} q\right)(x, D)$ and $\left[\partial_{x^{\prime \prime}}, q(x, D)\right]=$ $\left(\partial_{x^{\prime \prime}} q\right)(x, D)$ we can compute

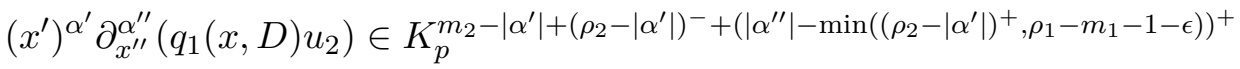

$$
\begin{aligned}
& \Rightarrow \quad\left(x^{\prime}\right)^{\alpha^{\prime}}\left(q_{1}(x, D) u_{2}\right) \in K_{p}^{m_{2}-\left|\alpha^{\prime}\right|+\left(\rho_{2}-\left|\alpha^{\prime}\right|\right)^{-}, \min \left(\left(\rho_{2}-\left|\alpha^{\prime}\right|\right)^{+}, \rho_{1}-m_{1}-1-\epsilon\right)} \\
& \Rightarrow \quad\left(q_{1}(x, D) u_{2}\right) \in K_{p}^{m_{2}, \min \left(\rho_{2}, \rho_{1}-m_{1}-1-\epsilon\right)} .
\end{aligned}
$$

Thus in the first case $u_{1} u_{2} \in K_{p}^{\max \left(m_{i}\right), \max \left(m_{i}\right)-\max \left(m_{i}-\rho_{i}\right)}$ and the second case follows from Taylor expansion. Note that $u_{1}=\left(x^{\prime}\right)^{N} \tilde{u}_{1}$ with $\tilde{u}_{1} \in K_{p}^{m_{1}+N}$ with the same proof as in Proposition 2.18, which together with the above computations on $\left(x^{\prime}\right)^{\alpha^{\prime}} q(x, D) u_{2}$ yields the claim.

The following Proposition is the main reason we introduce this space. In contrast to the $I_{1,1}^{m, \rho}$ space composition is bounded with respect to the Banach norm and not with seminorms differing from one another, enabling uniform boundedness results.

Proposition 3.6. Let $p \in I_{1,1}^{m_{1}, \rho_{1}}\left(\mathbb{R}^{n}, \mathbb{R}^{n-1}\right)$ with $m_{1}<-1$ and $\kappa$ be a diffeomorphism of $K_{p}^{m_{2}, \rho_{2}}$-type with $m_{2}<-2$ and, then

$$
\|p(\kappa)\|_{K_{p}^{m, \rho}} \leq C\left(\|\kappa\|_{\mathrm{ess}}\right)\|p\|\left(1+\|\kappa\|_{K_{p}^{m_{2}, \rho_{2}}}\right)
$$

for $m=\max \left(m_{1}, m_{2}\right)$ and

$$
m-\rho= \begin{cases}\max \left(m_{1}-\rho_{1}, m_{2}-\rho_{2}\right) & m_{1}+2<0 \\ \max \left(m_{1}-\rho_{1}, m_{2}-\rho_{2}+m_{1}+2\right) & m_{1}+2>0 \\ \max \left(m_{1}-\rho_{1}, m_{2}-\rho_{2}+\epsilon\right) & m_{1}+2=0\end{cases}
$$

Proof. Let $p_{s}$ be the smoothing of $p$ as usual and

$$
\kappa_{s}=\left(x^{\prime \prime}+\varphi\left(D_{x} / s\right) \kappa^{\prime \prime}(x), x^{\prime} \varphi\left(D_{x} / s\right) F\right) .
$$

Then

$$
\begin{aligned}
& p(\kappa)=p_{t}\left(\kappa_{t}\right) \\
& +\int_{t}^{\infty}\left[\psi\left(D_{x} / s\right) p_{G}+\psi\left(D_{x^{\prime}} / s\right) p_{C}\right] \circ \kappa_{s}+\left(\nabla p_{s}\right) \circ \kappa_{s} \cdot\left(\begin{array}{c}
x^{\prime} \psi\left(D_{x} / s\right) F \\
\psi\left(D_{x} / s\right) \kappa^{\prime \prime}
\end{array}\right) \mathrm{d} \ln (s) .
\end{aligned}
$$

Using Propositions 2.34 and 2.38, we obtain that the first two summands under the integral fulfill

$$
\int_{t}^{\infty}\left[\psi\left(D_{x} / s\right) p_{G}+\psi\left(D_{x^{\prime}} / s\right) p_{C}\right] \circ \kappa_{s} \mathrm{~d} \ln (s) \in I_{1,1}^{m_{1}, \min \left(\rho_{1},-m_{2}-2+\rho_{2}\right)} \subseteq K_{p}^{m, \rho}
$$


with each seminorm bounded by a product of some constant depending on the essential bounds $C_{1}, C_{2}, C_{3}$ and a seminorm of $p$, provided that $t \geq T$, where $T$ is the essential bound. For the last summand in the integral, we obtain from Lemma 2.32 the symbol

$q(x, \xi)=\int_{t}^{\infty}\left(\left(\nabla p_{s}\right) \circ \kappa_{s}\right) \psi(\xi / s) \mathrm{d} \ln (s) \in \begin{cases}S_{1,1}^{0,0, \min \left(-m_{1}-2+\rho_{1},-m_{2}-1+\rho_{2}\right)} & m_{1}<-2 \\ S_{1,1}^{-m_{1}-2,0, \min \left(\rho_{1}, m_{1}+1-m_{2}+\rho_{2}\right)} & m_{1}>-2 \\ S_{1,1}^{\epsilon, 0, \min \left(\rho_{1},-m_{2}-1+\rho_{2}\right)+\epsilon} & m_{1}=-2\end{cases}$

with each seminorm bounded by a product of some constant depending on the essential bounds $C_{1}, C_{2}$ and a seminorm of $p$. By commutation relations with $\partial_{x^{\prime \prime}}$ and $x^{\prime}$ we obtain that

$$
q(x, D): K_{p}^{m_{2}, \rho_{2}} \rightarrow K_{p}^{m, \rho}
$$

continuously, with operator norms bounded by symbol seminorms. Lastly choosing $t=T$ the first summand satisfies

$$
p_{t} \circ \kappa_{t} \in C_{b}^{\infty}
$$

with each seminorm bounded by a product of a seminorm of $p$ and some constant depending on $T$ and $C_{4}$. This yields the claim.

By virtue of our preparations in Section 4 also the following composition result is almost immediate.

Proposition 3.7. Let $\rho>0, m<-2$ and $f \in C_{*}^{-m+\rho}\left(\mathbb{R}^{d}\right)$ and $u=\left(u_{i}\right)_{i=1}^{d} \in$ $K_{p}^{m, \rho}\left(\mathbb{R}^{n}, \mathbb{R}^{n-1} ; \mathbb{R}^{d}\right)$, then

$$
f\left(u_{1}(x), \ldots, u_{d}(x)\right) \in K_{p}^{m, \rho}\left(\mathbb{R}^{n}, \mathbb{R}^{n-1}\right)
$$

Proof. Without loss of generality $f$ is compactly supported. Use the parallel construction for a smooth approximation as in Proposition 3.6

$$
\begin{aligned}
u_{i, s} & =\varphi(D / s) u_{i}, \quad u_{s}=\left(u_{i, s}\right)_{i=1}^{d}, \quad f_{s}=\varphi(D / s) f \\
f(u) & =\lim _{s \rightarrow \infty} f_{s}\left(u_{s}\right)=f_{t}\left(u_{t}\right)+\int_{t}^{\infty}(\psi(D / s) f) \circ u_{s}+\sum_{j=1}^{d} \partial_{j} f_{s}\left(u_{s}\right) \psi(D / s) u_{j} \mathrm{~d} \ln (s)
\end{aligned}
$$

We obtain the estimates on the first two summands - just as in Theorem $2.40-$ from Lemma 2.36. Now as

$$
\left|\partial_{x^{\prime \prime}}^{\alpha^{\prime \prime}}\left(\partial_{j} f\right)\left(u_{s}\right)\right| \lesssim s^{\left(m-\rho+1+\left|\alpha^{\prime \prime}\right|\right)_{\epsilon}^{+}}
$$

by chain rule, proceeding with a computation parallel to Lemma 2.32, we obtain for the symbol $q(x, \xi)$

$$
\left|\partial_{x}^{\alpha} \partial_{\xi}^{\beta} q(x, \xi)\right| \lesssim\langle\xi\rangle^{\left|\alpha^{\prime}\right|-|\beta|+\left(m-\rho+1+\left|\alpha^{\prime \prime}\right|\right)_{\epsilon}^{+}} .
$$

Which together with the commutation relations as in Proposition 3.5 yields the claim.

With these preparations we can solve the equation.

TheOREM 3.8. Let $m<-2$, with $m, \rho, m-\rho \notin \mathbb{Z}$ and $p, q$ as in (1). And let $\tilde{v} \in G_{0}^{m-\rho}\left(\mathbb{R}^{n-1}, \mathbb{R}^{n}\right)$ and $\tilde{g} \in C^{j}\left([0, T] ; G_{0}^{m-\rho+j}\left(\mathbb{R}^{n-1}, \mathbb{R}^{n}\right)\right)$ for all $m-\rho+j<-1$. Then

$$
\left\{\begin{array}{l}
\partial_{t} u+p \partial_{x} u+q u=\tilde{g} \\
u_{\left.\right|_{t=0}}=\tilde{v}
\end{array}\right.
$$


has a unique solution $u \in C^{j}\left([0, T] ; G_{0}^{m-\rho+j}\left(\mathbb{R}^{n-1}, \mathbb{R}^{n}\right)\right)$.

Proof. We will use the method of characteristics, as in the transport equation, to obtain for $1<p \leq \infty$

$$
\left\|\psi_{\nu}(D) u\right\|_{L^{p}} \lesssim 2^{\nu(m+1-1 / p)} .
$$

So define $\gamma(t, x ; \tau)$ to be the flow of the vector field from the equation, namely

$$
\left\{\begin{array}{l}
\frac{\mathrm{d}}{\mathrm{d} \tau} \gamma(t, x ; \tau)=(1, p(\gamma(t, x ; \tau))) \\
\gamma(t, x ; t)=(t, x)
\end{array}\right.
$$

For short times $|t-\tau|<T_{0}$, define the approximation series of the spacial component of $\gamma$ starting with $\gamma_{0}(t, x ; \tau)=x$

$$
\gamma_{N}(t, x ; \tau)=\int_{t}^{\tau} p\left(s, \gamma_{N-1}(t, x ; s)\right) \mathrm{d} s .
$$

It converges uniformly for all $t$ in $C^{1}\left(\left[t-T_{0}, t+T_{0}\right] ; C\left(\mathbb{R}^{n}\right)\right)$ to $\gamma$ via a Banach fix point argument. We have for every $N, \gamma_{N}=\left(\gamma_{N}^{\prime \prime}, x^{\prime} F_{N}\right)$.

By induction, we show for all $\left|\alpha^{\prime}\right|+m+1+\left(\left|\alpha^{\prime \prime}\right|-\rho\right)^{+}<0$, using the same expansion as in (6),

$$
\left.\mid \partial_{x}^{\alpha} \gamma_{N+1}(t, x ; \tau)\right)|\leq 1+| \int_{t}^{\tau} C\left(\|p\|,\left(\left|\partial_{x}^{\alpha_{1}} \gamma_{N}(t, x ; s)\right|\right)_{\left\{\alpha_{1}<\alpha\right\}}\right)\left(1+\left|\partial_{x}^{\alpha} \gamma_{N}(t, x ; s)\right|\right) \mathrm{d} s \mid .
$$

As in the expansion the highest degree derivative is only present once. These are uniformly bounded from Grönwall's lemma. We obtain boundedness of the respective Hölder norms of these derivatives, with the same argument.

Thus as $\gamma_{N}(t, x, \tau)$ for $|t-\tau|<T_{0}$ are small controlled perturbations of the identity, we obtain uniform essential bounds on their convex hull.

So by Proposition 3.6, we obtain

$$
\left\|\gamma_{N+1}(t, \cdot ; s)\right\|_{K_{p}^{m, \rho}} \leq \mid \int_{t}^{\tau} C\|p\|\left(1+\left\|\gamma_{N}(t, \cdot ; s)\right\|_{\left.K_{p}^{m, \rho}\right) \mathrm{d} s} \mid\right.
$$

yielding uniform boundedness of

$$
\left\|\gamma_{N}(t, \cdot ; \tau)\right\|_{K_{p}^{m, \rho}}<M
$$

Let $\gamma_{N, \lambda}=\lambda \gamma_{N+1}+(1-\lambda) \gamma_{N}$. We compute

$$
p\left(s, \gamma_{N}\right)-p\left(s, \gamma_{N+1}\right)=\int_{0}^{1}\left(\nabla_{x} p\right)\left(s, \gamma_{N, \lambda}\right) \cdot\left(\begin{array}{c}
\gamma_{N+1}^{\prime \prime}-\gamma_{N}^{\prime \prime} \\
x^{\prime}\left(F_{N+1}-F_{N}\right)
\end{array}\right)
$$

Now with Proposition 3.6, we obtain

$$
\begin{aligned}
\nabla_{x}^{\prime \prime} p\left(s, \gamma_{N, \lambda}(t, \cdot ; \tau)\right. & \in K_{p}^{m+(\rho-1)^{-},(\rho-1)^{+}} \\
\left(\partial_{x}^{\prime} p\right)\left(s, \gamma_{N, \lambda}(t, \cdot ; \tau)\right. & \in K_{p}^{m+1, \rho}
\end{aligned}
$$

with uniform bounds on norms. As $m+1<-1$ we can apply paramultiplication and obtain the estimate

$$
\left\|\left(\nabla_{x} p\right)\left(s, \gamma_{N, \lambda}\right) \cdot\left(\begin{array}{c}
\gamma_{N+1}^{\prime \prime}-\gamma_{N}^{\prime \prime} \\
x^{\prime}\left(F_{N+1}-F_{N}\right)
\end{array}\right)\right\|_{K_{p}^{m+(\rho-1)^{-},(\rho-1)^{+}}} \lesssim\left\|\gamma_{N+1}-\gamma_{N}\right\|_{K_{p}^{m+(\rho-1)^{-},(\rho-1)^{+}}}
$$

Thus $\gamma_{N}(t, \cdot ; \tau) \stackrel{\mathrm{s}-K_{p}^{m+(\rho-1)^{-},(\rho-1)^{+}} \longrightarrow}{\longrightarrow} \gamma(t, \cdot ; \tau)$ uniformly in $t, \tau$ with $|t-\tau|<T_{0}$. Now we have the estimates

$$
\left\|\varphi_{\nu}(D) u\right\|_{K_{p}^{m, \rho}} \leq C(\nu)\|u\|_{K_{p}^{m+(\rho-1)^{-},(\rho-1)^{+}}} \quad \limsup _{\nu \rightarrow \infty}\left\|\varphi_{\nu}(D) u\right\|_{K_{p}^{m, \rho}} \approx\|u\|_{K_{p}^{m, \rho}}
$$


which are immediate consequences of the Definition of $K_{p}^{m, \rho}$ in terms of unif $B_{p, \infty}^{s}$ spaces. Thus we can estimate

$$
\begin{aligned}
\|\gamma(t, \cdot ; \tau)\|_{K_{p}^{m, \rho}} & \leq \limsup _{\nu \rightarrow \infty}\left\|\varphi_{\nu}(D) \gamma\right\|_{K_{p}^{m, \rho}} \\
& \leq \limsup _{\nu \rightarrow \infty} \liminf _{N \rightarrow \infty}\left\|\varphi_{\nu}(D)\left[\gamma-\gamma_{N}\right]\right\|_{K_{p}^{m, \rho}}+\left\|\varphi_{\nu}(D) \gamma_{N}\right\|_{K_{p}^{m, \rho}} \\
& \leq \limsup _{\nu \rightarrow \infty} \liminf _{N \rightarrow \infty} C(\nu)\left\|\gamma-\gamma_{N}\right\|_{K_{p}^{m+(\rho-1)^{-},(\rho-1)^{+}}}+\left\|\varphi_{\nu}(D) \gamma_{N}\right\|_{K_{p}^{m, \rho}} \\
& \leq \limsup _{\nu \rightarrow \infty} \liminf _{N \rightarrow \infty}\left\|\varphi_{\nu}(D) \gamma_{N}\right\|_{K_{p}^{m, \rho}} \lesssim M .
\end{aligned}
$$

Thus we obtain boundedness of $\gamma \in K_{p}^{m, \rho}$. Having these estimates on the flow, we analyze

$$
\begin{cases}\frac{\mathrm{d}}{\mathrm{d} \tau} u(\gamma(t, x ; \tau))+q u(\gamma(t, x ; \tau)) & =\tilde{g}(\gamma(t, x ; \tau)) \\ u(\gamma(t, x ; 0)) & =\tilde{v}(\gamma(t, x ; 0)) .\end{cases}
$$

Let $U(\tau)=u(\gamma(t, x ; \tau)), Q(\tau)=q(\gamma(\tau)), G(\tau)=\tilde{g}(\gamma(t, x ; \tau))$ and $V=\tilde{v}(\gamma(t, x ; 0))$. Then by variation of parameters, we obtain

$$
\begin{aligned}
& A(\tau)=\int_{0}^{\tau} Q(s) \mathrm{d} s \in C^{1}\left(\left[-T_{0}, T_{0}\right] ; K_{p}^{m, \rho}\right) \\
& U(\tau)=e^{-A(\tau)}\left(\int_{0}^{\tau} G(s) e^{A(s)} \mathrm{d} s+V\right)
\end{aligned}
$$

Now applying Propositions 3.7 and 3.5, we obtain that $U(\tau) \in K_{p}^{m-\rho}$ vanishing of order $N$ for all $N<-m+\rho-1$ in $x^{\prime}=0$. As this holds for any $p$, we obtain

$$
\begin{aligned}
u(t, x)=u(\gamma(t, x ; t))=\left(x^{\prime}\right)^{N} \tilde{u}_{0}(t, x) & \in L^{\infty}\left(\left[0, T_{0}\right] ; G_{0}^{m-\rho}\left(\mathbb{R}^{n}, \mathbb{R}^{n-1}\right)\right) \\
\tilde{u}_{0}(t, x) & \in L^{\infty}\left(\left[0, T_{0}\right] ; G^{m-\rho-N, N}\left(\mathbb{R}^{n}, \mathbb{R}^{n-1}\right)\right)
\end{aligned}
$$

Using the equation, we obtain by induction that

$$
\begin{aligned}
\partial_{t} u(t, x)=\left(x^{\prime}\right)^{N-j} \tilde{u}_{j}(t, x) & \in L^{\infty}\left(\left[0, T_{0}\right] ; G_{0}^{m-\rho+j}\left(\mathbb{R}^{n}, \mathbb{R}^{n-1}\right)\right) \\
\tilde{u}_{j}(t, x) & \in L^{\infty}\left(\left[0, T_{0}\right] ; G^{m-\rho+N, N-j}\left(\mathbb{R}^{n}, \mathbb{R}^{n-1}\right)\right)
\end{aligned}
$$





\section{CHAPTER 4}

\section{Nonlinear Propagation of Conormality}

Another immediate application for conormal distributions of type $(1,1)$ are first order quasilinear equations. Though the explicit symbolic structure is not apparent in the proof of the following theorem, by using the symbol expansion formulas for Theorem 2.29 one can uncover explicit symbol formulas.

Theorem 4.1. For $v \in I_{1,1}^{m, \rho}\left(\Omega_{0}, \Sigma_{0}\right), m<-2$ and $q_{i}, g \in C^{\infty}$ with uniform bounds in $t, x$ for $U$ within compact sets. The problem

$$
\left\{\begin{array}{l}
\partial_{t} u+\sum_{i} q_{i}(t, x, u) \partial_{x_{i}} u=g(t, x, u) \\
u_{\mid=0}=v
\end{array}\right.
$$

has a unique short time solution $u \in I_{1,1}^{m, \rho}(\Omega, \Sigma)$, with $\Sigma$ the characteristic outflow of $\Sigma_{0}$.

Proof. Define the vector field

$$
X(t, x, U)=\partial / \partial t+\sum_{i} q_{i}(t, x, U) \partial / \partial x_{i}+g(t, x, U) \partial / \partial U .
$$

Thus take $\gamma(\tau ; t, x, U)$ to be the flow of this smooth vector field - uniquely existing for small times $\tau$ provided $U$ stays within a compact set. Also given $v$ as initial datum we obtain a solution $u$ with graph $\left\{\gamma(\tau ; 0, x, v(x)) \mid \tau \in[0, T], x \in \Omega_{0}\right\}$

$$
u: \gamma_{t, x}(\tau ; 0, x, v(x)) \mapsto \gamma_{U}(\tau ; 0, x, v(x))
$$

as long as this is a graph. Restricting to the surface $\Omega_{0}$, we take a $C_{*}^{m-\rho}$-chart

$$
\begin{array}{r}
\kappa_{0}: \mathbb{R}^{n-1} \times \mathbb{R} \rightarrow \Omega_{0} \\
\Sigma_{0}=\kappa_{0}\left(\mathbb{R}^{n-1}\right) .
\end{array}
$$

Now take $\tilde{v} \in G^{m-\rho}$ a maximally smooth extension of $v_{\left.\right|_{\Sigma_{0}}}$ via Proposition 2.15 and take

$$
\begin{array}{r}
\kappa:[0, T] \times \mathbb{R}^{n-1} \times \mathbb{R} \rightarrow \Omega \\
\kappa\left(t, y_{0}^{\prime \prime}, y_{0}^{\prime}\right)=\gamma_{t, x}\left(t ; 0, \kappa_{0}\left(y_{0}^{\prime \prime}, y_{0}^{\prime}\right), \tilde{v}\left(\kappa_{0}\left(y_{0}^{\prime \prime}, y_{0}^{\prime}\right)\right)\right) .
\end{array}
$$

Thus by Theorem 2.40 we have $\kappa \in G^{m-\rho}$. Further define

$$
\begin{array}{r}
\Gamma:[0, T] \times \mathbb{R}^{n-1} \times \mathbb{R} \rightarrow \Omega \\
\Gamma\left(t, y_{0}^{\prime \prime}, y_{0}^{\prime}\right)=\gamma_{t, x}\left(t ; 0, \kappa_{0}\left(y_{0}^{\prime \prime}, y_{0}^{\prime}\right), v\left(\kappa_{0}\left(y_{0}^{\prime \prime}, y_{0}^{\prime}\right)\right)\right.
\end{array}
$$

which by Theorem 2.40 obeys $\Gamma \in I_{1,1}^{m, \rho}\left([0, T] \times \mathbb{R}^{n},[0, T] \times \mathbb{R}^{n-1} ; \Omega\right)$. Then we obtain

$$
u \circ \kappa=(u \circ \Gamma) \circ\left(\Gamma^{-1} \circ \kappa\right) \text {. }
$$

As we have

$$
\begin{array}{r}
u \circ \Gamma\left(t, y_{0}^{\prime \prime}, y_{0}^{\prime}\right)=\gamma_{U}\left(t ; 0, \kappa_{0}\left(y_{0}^{\prime \prime}, y_{0}^{\prime}\right), v\left(\kappa_{0}\left(y_{0}^{\prime \prime}, y_{0}^{\prime}\right)\right)\right) \\
u \circ \Gamma \in I_{1,1}^{m, \rho}\left([0, T] \times \mathbb{R}^{n},[0, T] \times \mathbb{R}^{n-1}\right) .
\end{array}
$$


Further, as $\kappa^{-1} \in G^{m-\rho}$, which follows by Theorem 2.42 , we obtain by Theorem 2.29

$$
\begin{array}{r}
\Gamma\left(t, y_{0}^{\prime \prime}, y_{0}^{\prime}\right)=\gamma_{t, x}\left(t ; 0, \kappa_{0}\left(y_{0}^{\prime \prime}, y_{0}^{\prime}\right), v\left(\kappa_{0}\left(y_{0}^{\prime \prime}, y_{0}^{\prime}\right)\right)\right) \\
\kappa^{-1} \circ \Gamma \in I_{1,1}^{m, \rho}\left([0, T] \times \mathbb{R}^{n},[0, T] \times \mathbb{R}^{n-1} ;[0, T] \times \mathbb{R}^{n}\right) \\
\left(\kappa^{-1} \circ \Gamma\right)_{\left.\right|_{[0, T] \times \mathbb{R}^{n-1}}}=\operatorname{id}_{\left.\right|_{[0, T] \times \mathbb{R}^{n-1}}} .
\end{array}
$$

And thus we obtain by Theorem 2.42

$$
\begin{array}{r}
\Gamma^{-1} \circ \kappa \in I_{1,1}^{m, \rho}\left([0, T] \times \mathbb{R}^{n},[0, T] \times \mathbb{R}^{n-1} ;[0, T] \times \mathbb{R}^{n}\right) \\
\left.\left(\Gamma^{-1} \circ \kappa\right)\right|_{[0, T] \times \mathbb{R}^{n-1}}=\operatorname{id}_{\left.\right|_{[0, T] \times \mathbb{R}^{n-1}} .} .
\end{array}
$$

This gives us by Theorem 2.29

$$
\begin{array}{r}
u \circ \kappa \in I_{1,1}^{m, \rho}\left([0, T] \times \mathbb{R}^{n},[0, T] \times \mathbb{R}^{n-1}\right) \\
u \in I_{1,1}^{m, \rho}(\Omega, \Sigma) .
\end{array}
$$




\section{CHAPTER 5}

\section{Outlook}

The developed symbol calculus is suitable for applications in hyperbolic Partial Differential Equations, as we learned from our toy examples. From Theorem 4.1, we learned that the symbolic structure of solutions to first order hyperbolic quasilinear equations is stable under nonlinear perturbations from $G_{0}^{m-\rho}$. An interesting follow up question - apart from the analysis of higher order equations - is whether one can widen the term of being fully symbolic, such that no spreading into $G_{0}^{m-\rho, \infty}$ occurs for fully symbolic datum.

The natural occurrences of 'exotic symbols' as in the symbol reduction from Proposition 2.12 and the action of pseudodifferential operators on conormal distributions given by Theorem 2.14 imply that with the admission of 'exotic symbols' this is most likely the case.

If not then we at least expect the spreading to lie in a $x^{\prime \prime}$-trace admissible subspace $\tilde{G}^{\mu} \subseteq G^{\mu}$

$$
u \in \tilde{G}^{\mu} \Leftrightarrow\left\|\psi_{\nu}(D) u\right\|_{L^{p}\left(\mathbb{R}_{x^{\prime}}^{k} ; L^{\infty}\left(\mathbb{R}_{x^{\prime \prime}}^{n-k}\right)\right)} \lesssim 2^{-\nu(\mu-k(1-1 / p))} \quad \forall 1<p \leq \infty .
$$

This space also includes $I_{1,1}^{\mu}$ and distributions from exotic symbols as we can see from the Proof of 2.8. It might even be the case that a generalization of $\mid \mathbf{H o ̈ 0 7}$, Lemma 18.2.4.] yields a one to one relation between the exotic symbols and $\tilde{G}^{m, \infty}$.

Similar to the analysis of fully symbolic distributions, the treatment of the case $\ell=\infty$ will be interesting, as this is very close to the usual $I_{1,0}^{m}$ situation but allows for a singular geometry.

The unsatisfying cases of $S_{1,1}^{m, \rho^{\prime}, \rho^{\prime \prime}}$ with $\rho^{\prime} \in \mathbb{Z}$ should be improved by a more rigorous analysis of the behavior of our symbols near such cases and the implementation of $\left(\log \left\langle\xi^{\prime}\right\rangle\right)^{k}$ estimates. 

Appendices 



\section{CHAPTER A}

\section{Littlewood Paley decomposition and Besov spaces}

For the unfamiliar reader we briefly introduce some of the technical foundations to this thesis and refer to $\mid \mathbf{H o ̈ 0 3 b}$ or $\mid$ Tay91 for a more detailed discussion.

Definition A.1 (Discrete and continuous Littlewood-Paley). Let $\varphi \in C_{c}^{\infty}(\mathbb{R})$ with

$$
\begin{array}{ll}
\varphi(x)=1 & \forall|x| \leq 1 / 2 \\
\varphi(x)=0 & \forall|x| \geq 1
\end{array}
$$

then define on $\mathbb{R}^{n}$ for $\nu \in \mathbb{N}_{0}$

$$
\begin{aligned}
& \varphi_{\nu}(\xi)=\varphi\left(2^{-\nu}|\xi|\right) \\
& \psi_{\nu}(\xi)= \begin{cases}\varphi_{\nu+1}(\xi)-\varphi_{\nu}(\xi) & \nu \geq 0 \\
\varphi_{0}(\xi) & \nu=-1\end{cases}
\end{aligned}
$$

In an abuse of notation we can also define for $t \geq 1$ :

$$
\begin{aligned}
\varphi_{t}(\xi) & =\varphi(|\xi| / t) \\
\psi_{t}(\xi) & =t \frac{\mathrm{d}}{\mathrm{d} t} \varphi_{t}(\xi)=\psi_{1}(\xi / t)
\end{aligned}
$$

This yields the following discrete and continuous partitions of unity

$$
\begin{aligned}
& 1=\varphi_{\mu}(\xi)+\sum_{\nu=\mu}^{\infty} \psi_{\nu}(\xi)=\sum_{\nu=-1}^{\infty} \psi_{\nu}(\xi) \\
& 1=\varphi_{s}(\xi)+\int_{s}^{\infty} \psi_{t}(\xi) \mathrm{d} \ln (t)
\end{aligned}
$$

It will be clear from context, whether we address the discrete or the continuous partition of unity. In general their usage is almost equivalent and which to use is only a choice of convenience. For the most part the discrete version will have Greek subscripts and the continuous Latin, but there will be exceptions.

With this partition, we are able to define Besov-spaces.

Definition A.2. Let $1 \leq p, q \leq \infty$ and $s \in \mathbb{R}$, then the Besov space $B_{p, q}^{s}\left(\mathbb{R}^{n}\right)$ is defined as the closure of $C_{c}^{\infty}\left(\mathbb{R}^{n}\right)$ with the following norm

$$
\begin{aligned}
\|u\|_{B_{p, q}^{s}} & =\left\|\left(2^{\nu s}\left\|\psi_{\nu}(D) u\right\|_{L^{p}\left(\mathbb{R}^{n}\right)}\right)_{\nu}\right\|_{\ell^{q}\left(\mathbb{N}_{0} \cup\{-1\}\right)} \\
& \sim\left\|\varphi_{1}(D) u\right\|_{L^{p}\left(\mathbb{R}^{n}\right)}+\left\|t^{s}\right\| \psi_{t}(D) u\left\|_{L^{p}\left(\mathbb{R}^{n}\right)}\right\|_{L^{q}(1, \infty)}
\end{aligned}
$$

Important special cases are the Sobolev spaces $B_{2,2}^{s}=H^{s}$ and the Hölder-Zygmund spaces $B_{\infty, \infty}^{s}=C_{*}^{s}$.

Definition A.3. Let $E\left(\mathbb{R}^{n}\right) \subset D^{\prime}\left(\mathbb{R}^{n}\right)$ be a Banach space such that

$$
u \in E\left(\mathbb{R}^{n}\right), f \in C_{c}^{\infty}\left(\mathbb{R}^{n}\right), x \in \mathbb{R}^{n} \Rightarrow f u \in E\left(\mathbb{R}^{n}\right), u(\cdot-x) \in E\left(\mathbb{R}^{n}\right) .
$$


And let $\varpi \in C_{c}^{\infty}$ such that $1=\sum_{z \in \mathbb{Z}^{n}} \varpi(x-z)$. Then the uniform $E$ space can be defined as

$$
\text { unif } E=\left\{u \in D^{\prime}\left(\mathbb{R}^{n}\right) ;\|u\|_{\text {unif }}=\sup _{z \in \mathbb{Z}^{n}}\|\varpi(\cdot-x) u\|_{E}<\infty\right\}
$$

Especially interesting are $L_{\text {unif }}^{p}\left(\mathbb{R}^{n}\right)={ }_{\text {unif }}\left(L^{p}\right)\left(\mathbb{R}^{n}\right)$ and its derived spaces as a more strict version of $L_{\mathrm{loc}}^{p}$. We are going to give an alternate norm of unif $\left(B_{p, q}^{s}\right)\left(\mathbb{R}^{n}\right)$ using unif $\left(L^{p}\right)\left(\mathbb{R}^{n}\right)$ instead of $L^{p}\left(\mathbb{R}^{n}\right)$ in the definition of $B_{p, q}^{s}\left(\mathbb{R}^{n}\right)$. Therefore introduce the following notion.

Definition A.4. Let $a(x, D): S\left(\mathbb{R}^{n}\right) \rightarrow S^{\prime}\left(\mathbb{R}^{n}\right)$ be a weakly continuous linear operator with symbol $a(x, \xi)$. Then the convolution kernel $K$ of $a(x, D)$ is defined by the following oscillatory integral

$$
K(x, z)=\int e^{i z \xi} a(x, \xi) \mathrm{d} \xi
$$

and satisfies

$$
a(x, D) u=\int K(x, z) u(x-z) \mathrm{d} z
$$

Proposition A.5 (Almost locality I). Let $K_{\nu}(x, z)$ be the convolution kernel of $\psi_{\nu}(D)$, which is - as $\psi_{\nu}(D)$ is constant in $x$ just the Fourier transform of $\psi_{\nu}$

$$
K_{\nu}(x, z)=\int e^{i z \xi} \psi_{\nu}(\xi) d \xi
$$

Then we have for all $N \in \mathbb{N}_{0} \cup\{-1\}$ the uniform estimates

$$
\left|\partial_{z}^{\alpha} K_{\nu}(x, z)\right| \lesssim 2^{\nu|\alpha|}\left\langle 2^{\nu} z\right\rangle^{-N}
$$

Proof. Let $z \neq 0$, take $j$ a coordinate direction such that $\left|z_{j}\right|$ is maximal. By integration by parts, we obtain

$$
\begin{aligned}
\partial_{z}^{\alpha} K(x, z) & =\left(\frac{i}{z_{j}}\right)^{N} \int e^{i z \xi}(i \xi)^{\alpha} \partial_{\xi_{j}}^{N} \psi_{\nu}(\xi) \mathrm{d} \xi=\left(\frac{i}{2^{\nu} z_{j}}\right)^{N} \int e^{i z \xi}(i \xi)^{\alpha}\left(\partial_{\xi_{j}}^{N} \psi\right)_{\nu}(\xi) \mathrm{d} \xi \\
\left|\partial_{z}^{\alpha} K(x, z)\right| & \leq C_{N} 2^{\nu|\alpha|} \min \left(1,\left|2^{\nu} z_{j}\right|^{-N}\right) \lesssim 2^{\nu|\alpha|}\left\langle 2^{\nu} z\right\rangle^{-N}
\end{aligned}
$$

COROllary A.6. The following are equivalent norms on unif $B_{p, \infty}^{s}$.

$$
\begin{aligned}
& \|u\|_{1}=\sup _{z, \nu} 2^{\nu s}\left\|\psi_{\nu}(D) \varpi(\cdot-z) u\right\|_{L^{p}} \\
& \|u\|_{2}=\sup _{z, \nu} 2^{\nu s}\left\|\varpi(\cdot-z) \psi_{\nu}(D) u\right\|_{L^{p}}
\end{aligned}
$$

Proof. Consider

$$
\begin{aligned}
\varpi(\cdot-z) \psi_{\nu}(D) u & =\sum_{z^{\prime}} \varpi(\cdot-z) \psi_{\nu}(D) \varpi\left(\cdot-z^{\prime}\right) u \\
\psi_{\nu}(D) \varpi\left(\cdot-z^{\prime}\right) u & =\sum_{z} \varpi(\cdot-z) \psi_{\nu}(D) \varpi\left(\cdot-z^{\prime}\right) u
\end{aligned}
$$

Now as either of the initial estimates directly implies boundedness of $u \in \mathcal{S}^{\prime}$, we obtain from A.5 for $\left|z-z^{\prime}\right|$ large enough rapid decay in any $L^{p}$ norm of the above summands - uniform with respect to any factor $2^{-N \nu}$. Thus there are only finitely many relevant terms to be estimated and we obtain equivalence of the respective norms. 
Proposition A.7 (Almost locality II). Let $a \in C_{*}^{\rho}$ with support $K$ and $\epsilon>0$, then for $d(x)=\operatorname{dist}\left(x, K+B_{\epsilon}\right)$ we have

$$
\left|\psi_{\nu}(D) a(x)\right| \lesssim 2^{-\nu \rho}\left\langle 2^{\nu} d\right\rangle^{-M}\|a\|_{C_{*}^{\rho}}
$$

Proof. First the estimate is obvious if $d=0$, so let $x \notin K+B_{\epsilon}$. By partial integration we have the estimate

$$
\begin{aligned}
\psi_{\nu}(D) a(x) & =\int e^{i(x-y) \xi} \psi_{\nu}(\xi) a(y) \mathrm{d} y \\
& =\int e^{i(x-y) \xi}\left(\Delta^{2 N} \psi_{0}\right)\left(2^{-\nu} \xi\right)\left|2^{\nu}(x-y)\right|^{-4 N} a(y) \mathrm{d} y
\end{aligned}
$$

Now interpret $\left|2^{\nu}(x-y)\right|^{-4 N} a(y)$ as the new function, wherein $x$ is just a parameter. Then we can estimate the $C_{*}^{\rho}$ norm by $\left\langle 2^{\nu} d\right\rangle^{-4 N}\|a\|_{C_{*}^{\rho}}$. Thus we obtain

$$
\left.\left|\int e^{i(z-y) \xi}\left(\Delta^{2 N} \psi_{0}\right)\left(2^{-\nu} \xi\right)\right| 2^{\nu}(x-y)\right|^{-4 N} a(y) \mathrm{d} y \mid \lesssim 2^{-\nu \rho}\left\langle 2^{\nu} d\right\rangle^{-4 N}\|a\|_{C_{*}^{\rho}}
$$

Thus taking $z=x$ yields the claim.

\section{Hölder-Zygmund estimates}

Proposition A.8. Let $a \in C_{*}^{\rho_{1}}$ and $b \in C_{*}^{\rho_{2}}$ with $\rho_{i}>0$, then

$$
a b=a_{\chi}(x, D) b+b_{\chi}(x, D) a+a_{\Phi}(x, D) b
$$

with

$$
\begin{aligned}
\left\|a_{\chi}(x, D) b\right\|_{C_{*}^{\rho_{2}}} & \leq C\left(\rho_{2}\right)\|a\|_{L^{\infty}}\|b\|_{C_{*}^{\rho_{2}}} \\
\left\|b_{\chi}(x, D) a\right\|_{C_{*}^{\rho_{1}}} & \leq C\left(\rho_{1}\right)\|b\|_{L^{\infty}}\|a\|_{C_{*}^{\rho_{1}}} \\
\left\|a_{\Phi}(x, D) b\right\|_{C_{*}^{\rho_{2}}} & \leq C\left(\rho_{2}\right)\|a\|_{L^{\infty}}\|b\|_{C_{*}^{\rho_{2}}}
\end{aligned}
$$

Proof. All norms of $a_{\chi}(x, \xi) \in S_{1,1}^{0}$ are controlled by $\|a\|_{L^{\infty}}$ and $\Psi_{1,1}^{0}$ is continuous on $C_{*}^{\rho_{i}}$.

Corollary A.9. Let $a \in S^{m_{1}, \rho_{1}}$ and $b \in S^{m_{2}, \rho_{2}}$, then $a b \in S^{m_{1}+m_{2}, \min \left(\rho_{i}\right)}$

Proof. Use Proposition A.8

$$
\left|\psi_{\nu}(D)\left[a\left(x, \eta^{\prime}\right) b\left(x, \eta^{\prime}\right)\right]\right| \lesssim 2^{-r \nu}\left\langle\eta^{\prime}\right\rangle^{m_{1}+m_{2}+\left(r-\rho_{1}\right)^{+}}+2^{-r \nu}\left\langle\eta^{\prime}\right\rangle^{m_{1}+m_{2}+\left(r-\rho_{2}\right)^{+}}
$$

Proposition A.10 (Proposition 8.6.12 from $\left[\right.$ Hö03b). Let $F \in C^{N+1}(\mathbb{R})$ and let $u$ be a real valued function in $C_{*}^{\rho}$ for $\rho \in(0, N)$. Then $F(u) \in C_{*}^{\rho}$, and if $F(0)=0$, $|u| \leq M$ we have

$$
\|F(u)\|_{C_{*}^{\rho}} \leq C_{\rho, N} \sum_{j=0}^{N} M^{j} \sup \left|F^{(j+1)}\right|\|u\|_{C_{*}^{\rho}}
$$





\section{Bibliography}

[Bea83] Michael Beals, Self-spreading and strength of singularities for solutions to semilinear wave equations, Annals of Mathematics 118 (1983), no. 1, 187-214.

[Bon80] Jean-Michel Bony, Propagation des singularités pour les équations aux dérivées partielles non linéaires, Séminaire Équations aux dérivées partielles (Polytechnique) (1979-1980) (fr), talk:22. MR 600707

[Bon86] _ Second microlocalization and propagation of singularities for semi-linear hyperbolic equations, Hyperbolic Equations and Related Topics (Sigeru Mizohata, ed.), Academic Press, 1986, pp. $11-49$.

[Bou88] Gérard Bourdaud, Une algèbre maximale d'opérateurs pseudo-différentiels de type 1, 1, Séminaire Équations aux dérivées partielles (Polytechnique) (1987-1988), 1-17 (fr), talk:7. MR 1018179

[Chi72] C.-H. Ching, Pseudo-differential operators with nonregular symbols, Journal of Differential Equations 11 (1972), 436-447.

[DH72] J. J. Duistermaat and L. Hörmander, Fourier integral operators. ii, Acta Math. 128 (1972), $183-269$.

[Hö03a] Lars Hörmander, Distribution theory and fourier analysis, reprint of the 2. ed. 1990 ed., The analysis of linear partial differential operators, vol. 1, Springer, Berlin [u.a.], 2003, Originally publ. as Vol. 256 in the series: Grundlehren der mathematischen Wissenschaften in 1983 and 1990 and thereafter as a Springer Study Edition in 1990.

[Hö03b] _ Lectures on nonlinear hyperbolic differential equations, 1 ed., Mathématiques et Applications, Springer, 2003.

[Hö05] , Differential operators with constant coefficients, reprint of the 1983 ed ed., The analysis of linear partial differential operators, vol. 2, Springer, Berlin [u.a.], 2005, Originally publ. as vol. 257 in the series: Grundlehren der mathematischen Wissenschaften.

[Hö07] , Pseudo-differential operators, reprint of the 1994 ed. ed., The analysis of linear partial differential operators, vol. 3, Springer, Berlin [u.a.], 2007, Originally publ. as vol. 274 in the series: Grundlehren der mathematischen Wissenschaften.

[Hö09] - Fourier integral operators, repr. of the 1994 ed ed., The analysis of linear partial differential operators, vol. 4, Springer, Berlin [u.a.], 2009, Zuerst ersch. als: Grundlehren der mathematischen Wissenschaften ; 275.

[Kum81] Hitoshi Kumanogō, Pseudo-differential operators : Hitoshi kumano-go ; [translated by hitoshi kumano-go, remi vaillancourt, and michihiro nagase], english-language ed ed., MIT Press, Cambridge, Massachusetts, [1982], c1981, Updated translation of: Gibibun sayoso. 1974.

[Lax06] Peter D. Lax, Hyperbolic partial differential equations, Courant lecture notes in mathematics ; 14, Courant Inst. of Mathematical Sciences, New York, NY, 2006.

[MR85] Richard Melrose and Niles Ritter, Interaction of nonlinear progressing waves for semilinear wave equations, Annals of Mathematics 121 (1985), no. 1, 187-213.

[Mé08] Guy Métivier, Para-differential calculus and applications to the cauchy problem for nonlinear systems, CRM series ; 5, Edizioni della Normale, Pisa, 2008, University lectures.

[Rau79] Jeffrey Rauch, Singularities of solutions to semilinear wave equations, Journal de Mathématiques Pures et Appliquées 58 (1979), 299 - 308.

[Ree78] Michael C. Reed, Propagation of singularities for non-linear wave equations in one dimension, Communications in Partial Differential Equations 3 (1978), no. 2, 153-199.

[RR80] Jeffrey Rauch and Michael C. Reed, Propagation of singularities for semilinear hyperbolic equations in one space variable, Annals of Mathematics 111 (1980), no. 3, 531-552.

[Shu01] Michail A. Shubin, Pseudodifferential operators and spectral theory, 2. ed., Springer, Berlin, 2001, kart. : DM 79.00. 
[Tay91] Michael Eugene Taylor, Pseudodifferential operators and nonlinear pde, 1. ed., Progress in Mathematics, Birkhäuser Boston, 1991.

[Tay00] _ Tools for pde : pseudodifferential operators, paradifferential operators, and layer potentials, Mathematical surveys and monographs ; 81, American Mathematical Society, Providence, RI, 2000.

[Tay11a] _ Partial differential equations : Basic theory, 2. ed., Partial differential equations, vol. 1, Springer, New York, [2011].

[Tay11b] _ Partial differential equations : Nonlinear equations, 2. ed., Partial differential equations, vol. 3, Springer, New York, [2011].

[Tay11c] _ Partial differential equations : Qualitative studies of linear equations, 2. ed., Partial differential equations, vol. 2, Springer, New York, [2011]. 